\title{
EVALUATION OF SOLID STATE ACCELEROMETER SENSOR FOR EFFECTIVE POSITION ESTIMATION
}

\author{
by \\ Meenal A. Lele \\ Submitted in partial fulfilment of the requirements \\ for the degree of Master of Applied Science \\ at \\ Dalhousie University \\ Halifax, Nova Scotia \\ November 2010
}

C Copyright by Meenal A. Lele, 2010 


\section{DALHOUSIE UNIVERSITY}

\section{Department of Electrical and Computer Engineering}

The undersigned hereby certify that they have read and recommend to the Faculty of Graduate Studies for acceptance a thesis entitled "EVALUATION OF SOLID STATE ACCELEROMETER SENSOR FOR EFFECTIVE POSITION ESTIMATION" by Meenal A. Lele in partial fulfillment of the requirements for the degree of Master of Applied Science.

Dated: November 22, 2010

Supervisor:

Dr. Jason Gu

Readers:

Dr. Jose Gonzalez Cueto

Dr. William J. Phillips 


\title{
DALHOUSIE UNIVERSITY
}

DATE: November 22, 2010

\begin{abstract}
AUTHOR: Meenal A. Lele
TITLE: $\quad$ EVALUATION OF SOLID STATE ACCELEROMETER SENSOR FOR EFFECTIVE POSITION ESTIMATION

DEPARTMENT OR SCHOOL: Department of Electrical and Computer Engineering DEGRE: $\quad$ M.A.Sc CONVOCATION: May $\quad$ YEAR: 2011 Permission is herewith granted to Dalhousie University to circulate and to have copied for non-commercial purposes, at its discretion, the above title upon the request of individuals or institutions. I understand that my thesis will be electronically available to the public.
\end{abstract}

The author reserves other publication rights, and neither the thesis nor extensive extracts from it may be printed or otherwise reproduced without the author's written permission.

The author attests that permission has been obtained for the use of any copyrighted material appearing in the thesis (other than the brief excerpts requiring only proper acknowledgement in scholarly writing), and that all such use is clearly acknowledged.

Signature of Author 


\section{Table of Contents}

$\begin{array}{ll}\text { List of Tables } & \text { vii }\end{array}$

List of Figures viii

$\begin{array}{lc}\text { List of Abbreviations } & \mathbf{x}\end{array}$

Acknowledgement $\quad$ xi

$\begin{array}{ll}\text { Abstract } & \text { xii }\end{array}$

1. Introduction 1

1.1 Measurement Uncertainty and Sensor Error Sources 4

1.2 Research Motivation 5

1.3 Research Contribution 5

$\begin{array}{lll}1.4 & \text { Research Significance } & 6\end{array}$

$\begin{array}{lll}1.5 & \text { Thesis Outline } & 7\end{array}$

$\begin{array}{ll}\text { 2. Robot Localization } & 8\end{array}$

2.1 Position Techniques 8

2.1.1 Relative Positioning 9

2.1.2 Absolute Positioning 10

$\begin{array}{ll}2.2 \text { Inertial Sensor Fusion } & 10\end{array}$

2.3 Acceleration as an Inertial Navigation Sensor 11

2.4 Factors Affecting Performance of Acceleration Sensor 12 
2.4.1 Bias Drift Error $\quad 13$

2.4.2 Scale Factor Error 14

2.4.3 Mechanical Vibration and Alignment 15

2.4.4 Cross Axis Sensitivity 15

2.4.5 Power Supply Variation 16

2.5 Acceleration Error Model 16

2.6 Previous Work 18

3. Testing Program, Methods and Procedures 21

3.1 Evaluation of MEMS Accelerometer 21

3.1.1 Principle of MEMS Accelerometer 21

3.1.2 Acceleration Sensor Mounting 22

3.2 Mechanical System Design 23

3.2.1 Accelerometer Evaluation-ADXL335 25

3.2.2 NiDAQ - USB 6009 Card 26

3.2.3 Rotating Wheel Arrangement 29

3.2.4 Slip Ring Arrangement 30

3.3 Four Point Tumble Test 31

4. Software System Design 32

4.1 Theory and Implementation of Kalman Filter 32

4.1.1 Kalman Filter Algorithm. 34

4.1.2 Kalman Gain 36

4.1.3 Kalman Filter Extension 37

4.1.4 Noise Covariances Tuning 40

4.1.5 Kalman Filter Smoothing 43

4.2 Sensor Fusion Using Kalman Filter 45

4.2.1 Position-Velocity-Acceleration Model 45

4.3 Bias Drift Error Model Using Kalman Filter 48 
5. Results and Discussions

5.1 Accelerometer Bias Error Model Test 55

5.1.1 Static Bias Test 55

5.1.2 Angular Bias Test 57

$\begin{array}{lll}5.2 \text { Simulation Model } & 61\end{array}$

5.3 Effect of Q and R 61

5.4 Linear Acceleration Measurement 64

$\begin{array}{lll}\text { 5.2.1 Roll Measurement } & 65\end{array}$

$\begin{array}{lll}\text { 5.2.2 Pitch Measurement } & 68\end{array}$

$\begin{array}{lll}5.5 & \text { Tilt Measurement } & 71\end{array}$

5.6 Angular Position Estimation 71

$\begin{array}{lll}5.7 & \text { Summary of Results } & 72\end{array}$

6. Conclusion and Recommendations

$\begin{array}{lll}6.1 \text { Conclusion } & 73\end{array}$

$\begin{array}{lll}6.2 & \text { Recommendations } & 74\end{array}$

$\begin{array}{ll}\text { Appendices } & \mathbf{7 5}\end{array}$

A.1 Sensor Fusion- Two Dimensional Error Model 75

$\begin{array}{lll}\text { A.2 Motor Speed Estimation } & 78\end{array}$

$\begin{array}{lll}\text { A.2.1 Technical Specifications } & 80\end{array}$

$\begin{array}{lll}\text { A.3 Mechanical Design } & 82\end{array}$

$\begin{array}{lr}\text { Bibliography } & 83\end{array}$ 


\section{List of Tables}

2.1 Accelerometer Bias Error Compensation 14

4.1 Levenberg Marquardt Fitted Parameters 50

5.1 ADXL335 Bias and Scalar Factor Parameters 61

$\begin{array}{lll}\text { A.1 } & \text { RPM Settings } & 79\end{array}$ 


\section{List of Figures}

2.1 Classification of Mobile Navigation 9

2.2 Sensor Fusion Classification 11

3.1 Accelerometer Sensing Principle 22

3.2 Accelerometer Sensor Mounting 23

3.3 Hardware Setup Block Diagram 24

3.4 NiDAQ USB 6009 -LabVIEW Interfacing 25

3.5 ADXL 335 Block Diagram 26

3.6 USB 6009 Block Diagram 27

3.7 MATLAB Functions Call From LabVIEW 28

3.8 Hardware Test Setup 29

3.9 Mercotac Electrical Connector 30

3.10 Four Point Tumble Test 31

4.1 Kalman Filter Block Diagram 35

4.2 Kalman Filter Algorithm 38

4.3 Covariance Time Dependency 42

4.4 Optimal Estimation Characteristics 43

4.5 Accelerometer Bias Data with Levenberg Marquardt Fitted Curve 50

4.6 Autocorrelation of Residuals 51

5.1 Mechanical Design 56

5.2 Static Bias Test (Data Down Sampled by 25) 56

5.3 Bias Drift Estimated by Kalman Filter 57

5.4 Output Response Vs. Orientation 58 
5.5 Ideal and Measured Fused Data of X Axis with Power Supply=3.3 Volts 59

5.6 Ideal and Measured Fused Data of Y Axis with Power Supply=3.3 Volts 59

5.7 Simulated Acceleration Data $\mathrm{Q}>\mathrm{R} \quad 62$

5.8 Kalman Gain and Error Covariance Q $>$ R 63

5.9 Simulated Acceleration Data $\mathrm{Q}<\mathrm{R}$

5.10 Kalman Gain and Error Covariance Q $<$ R 64

5.11 Rotating Wheel with Electrical Connector 65

$5.12 y_{1}, y_{2}$ Fused Acceleration Data 66

5.13 Position Estimation $y_{1}, y_{2}$ Data Fusion 67

5.14X axis Acceleration data 68

5.15 Position Estimation $x_{1}, x_{2}$ Data Fusion 69

$\begin{array}{lll}5.16 & \text { Kalman Gain } & 70\end{array}$

5.17 Unbounded Integrated Error and Smoothed Estimated Error at Different $\begin{array}{ll}\text { Speeds } & 70\end{array}$

5.18 Acceleration at Speed of $11 \mathrm{rpm} \quad 71$

$\begin{array}{ll}5.19 & \text { Angular Estimation }\end{array}$ 


\section{List of Abbreviations Used}

DR: Dead Reckoning

GPS: Global Positioning System

EKF: Extended Kalman Filter

KF: Kalman Filter

INS: Inertial Navigation System

NiDAQ: National Instruments Data Acquisition System

PSD: Power Spectrum Density

RTS: Rauch-Tung-Striebel

MEMS: Micro Electro Mechanical System

USB: Universal Serial Bus

PVA: Position-Velocity-Acceleration 


\section{ACKNOWLEDGEMENT}

The author would like to acknowledge NSERC (Natural Sciences Engineering Research Council, Canada) for the funding support for this research. The author would like to acknowledge the guidance and motivation received from her supervisor, Dr. Jason Gu of the Department of Electrical and Computer Engineering at Dalhousie University. The author would also like to thank Dr. Jose Gonzalez-Cueto of the Department of Electrical and Computer Engineering at Dalhousie University and Dr. William Philips of the Department of Engineering Mathematics at Dalhousie for their participation in the finalization of this thesis.

The author would like to thank all of her colleagues in the Robotics Research Groups and Progressive Engineers Co. (Mumbai) for their advice and support. Finally author would like to take this opportunity to thank her parents, husband and friends for their support. 


\begin{abstract}
Inertial sensors such as Gyroscope and Accelerometer show systematic as well as random errors in the measurement. Furthermore, double integration method shows accumulation of error in position estimation due to inherent accelerometer bias drift. The primary objective of this research was to evaluate ADXL 335 acceleration sensor for better position estimation using acceleration bias drift error model. In addition, measurement data was recorded with four point rotation test for investigation of error characteristics. The fitted model was validated by using nonlinear regression analysis. The secondary objective was to examine the effect of bias drift and scale factor errors by introducing error model in Kalman Filter smoothing algorithm. The study showed that the accelerometer may be used for short distance mobile robot position estimation. This research would also help to establish a generalized test procedure for evaluation of accelerometer in terms of sensitivity, accuracy and data reliability.
\end{abstract}




\section{CHAPTER 1}

\section{Introduction}

Mobile Robotics is one of the fastest growing technologies in today's world. In recent decades, mobile robotics has undergone significant technological advancements. With the development of nanotechnology, size of the robot has reduced considerably. Robotics Institute of America defines the term Robot as, "A reprogrammable, multifunctional manipulator designed to move material, parts, tools, or specialized devices through various programmed motions for the performance of a variety of tasks". The word Robot was derived from Czech word, "Robota" meaning forced labor. It was introduced by Czech play writer Karel Capek for the first time. Furthermore, Isaac Asimov used the word, Robotics in his short story about a robotherapist [1][2]. Since then, the term "Robotics" was commonly used to describe this technology.

The development started in $20^{\text {th }}$ century when Dr. W.Grey Walter invented tortoiseshaped robot which was controlled by simple DC motors and vacuum tubes. In fact, the first industrial robot was installed by General Motors called as Unimate. This robot was designed for material handling tasks such as lifting and stacking hot iron parts at appropriate locations. However, the major milestone in robotics was the development of "Shakey" by Stanford Research institute with computer vision, laser range finder and inertial sensors. The robotic arm developed by Stanford University in 1969 was used as a standard for robotic arm in research and education purposes. Presently, applications of robot are not only limited to industrial uses but also in service and entertainment areas. Autonomous robots are manufactured for navigating themselves in hostile environment. Use of autonomous robots in adverse environment has a greater benefit for application areas such as military, space, and research. In addition, autonomous under water vehicles are used for marine biology study, inspection, maintenance and cleaning operations. 
Underwater robots are of biologically inspired shape used to collect the data from underwater surface. Robot manipulators designed with movable arm are used for space exploration missions in the form of planetary rover, landers and pathfinders to gather data and explore the surface planet. Furthermore, robots are widely used in hospitals for transporting medical supplies and distribution of goods. Intelligent wheel chair assists handicap persons in mobility on unequal terrain or even use to climb stairs. Additionally, Transportation in warehouses made easy with the help of intelligent robots for material handling, lifting heavy loads and distribution. Robots such as Roomba proved an effective vacuum cleaner which senses the size of the room and changes the algorithm accordingly for maximum coverage. Robots are widely used in gaming applications as a toy with various sensors and wheels for locomotion. A tour-guide robot such as RoboX [3] is developed for museum, parks, exhibitions and other events to guide the visitors. Navigating mobile robots for such complex tasks, precise modelling and intelligent control is very important.

Navigation Problem:

To navigate a mobile robot in an environment, it must have a model which includes knowledge about its surroundings. Also, it has to analyze and distinguish the surroundings to find its position co-ordinates. Based on map matching it should perform appropriate action.

Thus, for determining position, a robot has to do the following tasks [4];

- Self-Localisation: The robot must know its current position before start to navigate in its surroundings.

- Goal Recognition: Robot must know where it is going. In other words, it should set the goal and move in appropriate direction.

- Path planning: after setting the goal, robot must know how to get there. This is known as path planning.

The main two tasks in mobile robot navigation are to acquire data from various sensors and interpreting the data by using behaviour based and map based techniques. The 
primary issues in robot navigation are computational power, failure to recognize objects, and failure to interpret data obtained from sensors [5]. Following points briefly discuss these issues.

- Computational Complexity: Computational burden increases depending upon the robotic applications. For real time systems, processing unit has to perform tedious calculations within a second which further needs computational power.

- Landmark Recognition: Without object information, it becomes hard to process the available information to estimate the position. It also takes more time to realise the environment in absence of landmarks. For outdoor applications, Object recognition may not be possible every time because of wind, fog, lightening etc. Furthermore, detailed knowledge of structures in the database is inevitable. Matching the objects with stored information is again a difficult job.

- Obstacle avoidance: Path planning technique is used to reach the goal in a collision free manner. Dynamic obstacles such as moving vehicles demands prior knowledge of moving obstacles and their trajectories [5].

- Sensor Fusion: Sensor fusion is to combine the data from different sensor for precise estimation. There is a possibility that the information coming from one of the sensor is not correct which needs to be resolved by perfect modelling.

- Sensor Failure: Sensors often fail to perform satisfactorily under real measurement conditions. Sensor output is degraded by internal as well as external error sources. Thus, for reliable sensor operation, sensor error sources should be minimised. 


\subsection{Measurement Uncertainty and Sensor Error Sources}

Determining the current position of the mobile platform by using previous position along with heading information over given time is called as dead reckoning. The most common dead reckoning sensors used for navigation are Odometry and inertial sensors. Odometry measures the wheel rotations by using sensors such as encoders to calculate position and direction. In addition to unrestrained accumulation of errors observed in Odometry, sources of errors can be categorized into two groups, systematic and random errors [6]. Systematic errors such as unequal wheel diameter, wheel misalignment and finite resolution of encoders and random errors such as long uneven trajectories, wheel spillage, accelerated sudden turns cause large position errors. Reference [7] focused on minimizing these errors by methods such as frequent calibration, and correction algorithms. Nevertheless, reduction of these types of errors is a challenging process since most of the errors cannot be predicted. By using external guiding sensors such as GPS, radar, active beacons and computer vision, unbounded odometry error can be minimised. By using the information obtained by additional sensors, the errors in odometry can be identified and corrected. However, additional sensors also show their own drawbacks. GPS and other wireless techniques faces some challenges such as signal blockage problems when came across tall buildings, military jamming place, radar or other radio frequency signals. Image distortion, failure in landmark recognition can cause errors in determining position.

The primary sensors used in mobile robot navigation are gyroscope and accelerometer. Integration of tilt or angular data from gyroscope gives the angular velocity while position can be roughly estimated by double integration of the acceleration data. The prominent disadvantage of using integration on these inertial sensors shows accumulation of errors and in long term duration the estimated position would be incorrect as compared to true position. In order to fuse the inertial sensor data with GPS, complete understanding of these errors is necessary [8]. Modelling the error sources in filter- 
processing algorithm is more efficient since low cost MEMS sensors show poor repeatability and need frequent calibration procedure[9] [10].

\subsection{Research Motivation}

The above discussion indicates that accuracy can be increased through frequent calibration of sensors and by using proper error compensation techniques. It drives a need of developing a calibration procedure and including errors in the process model when dealing with real time robot navigation.

Efficient state estimation is very crucial for computing robot's current location based on the previous location. The popular technique to integrate the inertial sensors information for position estimation is Kalman filter. Kalman filter is an optimal data processing algorithm with recursive characteristics [11] [12]. The algorithm uses all the available information about the measurement and based on system knowledge it provides the best possible solution. If the information obtained by sensors is poor or untrustworthy, it becomes difficult to rely on acquired data to predict the position. Thus, in-depth knowledge of sensor error contribution is required for building appropriate error models. This drives an essential need to develop reliable, error compensated model which is capable of providing low cost, short duration, and precise position data.

\subsection{Research Contribution}

The prime objective of this research was to obtain a comprehensive evaluation of accelerometer sensor errors for better position estimation. It was expected that the proposed research program would provide valuable results that will not only reduce accelerometer error contribution in position estimation but also provide a thorough understanding of MEMS accelerometer as an effective, low cost inertial sensor. 
The research project was elaborated to incorporate the following objectives:

- Determination of the effect of various error sources on Analog Device's ADXL 335 accelerometer sensor performance.

- Development of error models that describe behaviour of ADXL 335 accelerometer sensor by testing and calibrating the sensor on a mechanical rotation platform.

- Development and evaluation of the Kalman filter algorithm in MATLAB simulations as well as on the hardware set up to test the performance of error compensated accelerometer model.

- Model tuning by adjusting random noise parameters such as process noise covariance and measurement noise covariance matrix. Accelerometer sensor fusion in the Extended Kalman Filter for determination of position error.

- Incorporation of RTS smoothing algorithm for improved position estimation.

- Evaluation of testing parameters that affect the error reduction performance of ADXL 335 sensor in an effort to develop recommendations to standardize the error reduction testing and calibrating procedures.

\subsection{Research Significance}

This research was conducted on a commercially available ADXL 335 accelerometer sensor manufactured by Analog Devices which is used extensively in many robotics applications. In addition to the evaluation of error characteristics of accelerometer sensor, many other aspects of Kalman filtering algorithm have also been examined. Contributions of significance are summarized below:

- This study evaluated the effect of bias drift error and scale factor error on the performance of ADXL 335 sensor in terms of sensitivity, accuracy and data reliability. 
- Bias drift error model established through this research will allow the design and calibration of accelerometer sensor for applications such as aircraft roll and pitch measurement, mobile robot platform position estimation etc.

- The research will verify whether error model will significantly improve position estimation using acceleration sensor.

- The design parameters used for fitting bias accelerometer data exposed to rotation platform testing will be used as a deterministic input in Kalman filter algorithm.

- Mechanical Test rig designed for calibrating and testing ADXL 335 will help to establish a standard, generalised test procedure for evaluation of inertial sensors.

\subsection{Outline of Thesis:}

Thesis is arranged in the following sequence. Chapter 2 describes the concept of dead reckoning and sensor errors contribution. It also discusses sensor fusion technique used in inertial navigation. Furthermore, the chapter presents a literature review of basic error compensation techniques used in state estimation of mobile platforms and concludes with the proposed method for the same. Chapter 3 evaluates accelerometer for error characteristics. Additionally, there is a discussion about accelerometer principle and sensor mounting as well as description about the mechanical design system aspects. Chapter 4 provides an overview of the software system design used to implement the proposed system. The Kalman filter algorithm is discussed in detail along with bias error drift model. In Chapter 5, simulation results are included in first section followed by results obtained using proposed method on linear and angular acceleration measurement performed on hardware setup. Finally, in chapter 6, the effectiveness of accelerometer for short distance measurement is discussed along with future scope of work. Appendix contains pin configuration of acceleration sensor, NiDAQ card configuration and DC motor speed adjustments along with Dayton motor product data sheets. 


\section{CHAPTER 2}

\section{Robot Localization}

This chapter outlines a review of established research literature on accelerometer error reduction and position estimation methods used. First, positioning techniques and its classification is discussed. This is followed by a description of inertial sensor fusion and data fusion algorithm used. An overview of various error parameters that affect the performance of inertial sensors is also presented. Furthermore, a description of statistical error analysis used to "fit" the bias measurement data is also provided. Lastly, the chapter highlights published research work on the evaluation of accelerometer sensor, with an emphasis on error reduction techniques and calibration procedure used by previous researchers.

\subsection{Positioning Techniques}

Navigation is an important and critical topic of discussion in the field of mobile robotics. Locating current position compared with earlier position by means of various sensory data is evaluated in inertial navigation systems. Navigation can be described as, "The ability to establish its own position and orientation within a frame of reference" [13]. Starting with positioning of galaxy to very sophisticated intelligent systems, navigation methods have developed considerably in last five decades. Positioning techniques can be broadly categorized as, absolute positioning and relative positioning [14] [15] as discussed below; 


\subsubsection{Relative Positioning}

The position can be predicted by using the knowledge of past and present co-ordinates of the system. This method is commonly known as dead reckoning and the group it covers is called as relative position measurement [16]. Relative position measurement can be further classified into two groups; Odometry and Inertial navigation. Odometry contains different types of encoders are used to measure wheel rotation. In turn, encoders convert wheel rotation into linear displacement. Conversely, inertial navigation system is an integral part of the position estimation from $19^{\text {th }}$ century and now it is used in almost every tracking application such as missile control, spacecraft, marine navigation and underwater vehicle control. It consists of minimum three accelerometers and three gyroscopes collectively called as inertial measurement unit (IMU) to estimate position coordinates.

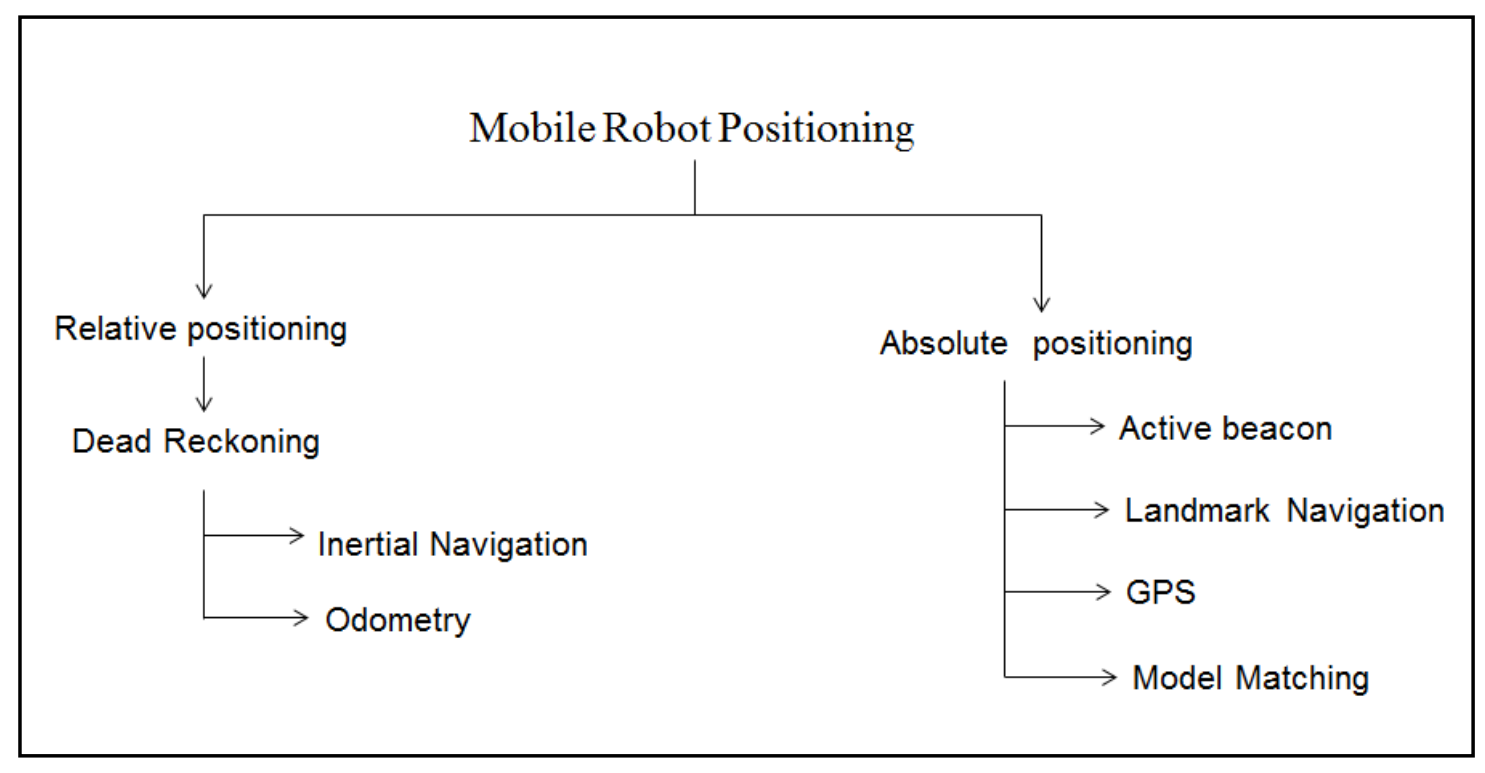

Figure 2.1 Classification of Mobile Robot Positioning Techniques 


\subsubsection{Absolute Positioning}

Absolute positioning covers landmark based and map based measurements such as active beacons, artificial and natural landmark recognition and model matching. GPS uses the model matching technique in which sensory data is compared with the global co-ordinate system for position estimation. The advantage of using this technique is that there is no accumulation of errors. Active beacon technique uses three or more transmitters and a rotary sensor to determine angle between the transmitters and mobile platform's axis. This method is called as triangulation. Landmark based absolute positioning can be further subdivided into Natural and Artificial landmark navigation. Computer vision technique is used to recognise a landmark. In model matching, the raw data obtained from sensors is interpreted for building local map. Furthermore, the map is matched with the database containing the global map. By combining sensors from both the methods, optimal position estimation can be achieved.

\subsection{Inertial Sensor Fusion}

In the last two decades there has been considerable development in the field of navigation especially when MEMS technology was introduced. Necessity of robust, autonomous and accurate tracking in military services such as missile control, marine war ships and aircrafts was the prime factor in the development of inertial navigation system. Collecting data from more than one sensor greatly increases the accuracy of the measurement. Integrating data from sensor of same kind or different types of sensors is called as "Sensor Fusion". Previous research [17] identifies three levels of sensor fusion models, data level, feature level and decision level as shown in figure 2.2. Data level combines sensory information and performs data adaptation. It contains probabilistic models for unit adjustments and batch estimation [18]. Kalman filter algorithm is used at data level to combine sensory information for further processing. Feature level performs feature extraction of each sensory measurement. The data is classified and stored in a cluster set 
based on pattern recognition and neural networks techniques. Decision level uses intelligent systems such as artificial intelligent and expert systems to fuse independent, pre-processed sensory data.

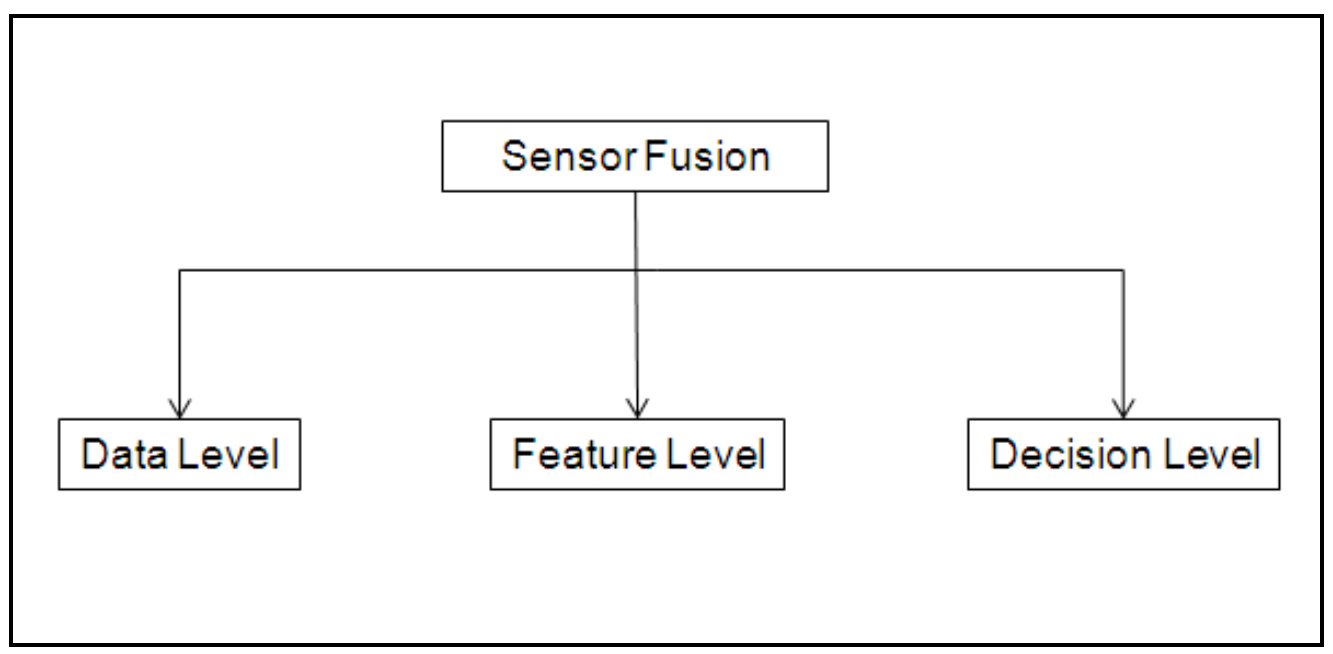

Figure 2.2 Sensor Fusion Classification

A more sophisticated inertial navigation system can be designed with the help of multiple sensors for determining the locations such as encoders for positioning, gyrocompass for orientation, tactile sensors to avoid obstacles, and accelerometers for measuring acceleration. Most of the dead reckoning systems use inertial sensors such as gyroscope accelerometer and magnetometer as a primary measurement along with external reference sensors such as GPS [14] for complete, accurate position estimation. Inertial Measurement unit (IMU) consists of at least three gyroscopes and three accelerometer sensors to completely define the three dimensional environment. IMU system has its own position errors that grow with time and trajectory.

\subsection{Accelerometer as an Inertial Navigation Sensor}

Acceleration sensing is an essential part of any Inertial Navigation system. Accelerometers are considered as one of the most important device in vibration and 
seismic load measurements as well as tilt and impact measurements. Accelerometers are comparatively low cost, easy mounting than the encoders and gyroscopes.

Newton laws of motion states the relationship between position, velocity and acceleration by following equations,

$$
\begin{aligned}
& x_{k}=x_{k-1}+v_{k-1} d t+a_{k-1}\left(d t^{2}\right) / 2 \\
& v_{k}=v_{k-1}+a_{k-1} d t \\
& a_{k}=a_{k-1}
\end{aligned}
$$

Position is obtained by double integrating accelerometer sensor data. $x_{k}$ represents position at time index $k, v_{k}$ represents velocity and $a_{k}$ as acceleration. Accumulation of error has been observed due to inherent accelerometer bias drift. In position estimation and tracking, use of accelerometer has been suggested by many researchers. Research [19-26] suggests that accelerometer can be used for estimating position for short duration and with low frequency operation. Accelerometer also shows major contribution in incorrect position estimation due to integral sources of errors such as mechanical imperfections, bias drifts, cross axis sensitivity etc. As wireless sensors are not available continuously as external reference sensor, error modeling of these sensors is inevitable.

\subsection{Factors Affecting Performance of Accelerometer Sensor}

The MEMS accelerometer has many advantages as compared to earlier accelerometers. These advantages include low cost, better performance, compact, and multi axis sensing on the same silicon wafer with inbuilt signal conditioning electronics. However, the sensor shows some errors in the measurement such as dynamic and static errors. Dynamic errors include measurement noise. Measurement noise has high frequency content and shows more peaks in power spectral density. To block such high frequency content, the 
bandwidth of acceleration sensor was set at $50 \mathrm{~Hz}$. Static errors include error characteristics within the sensor. As referenced in IEEE-STD-1293-1998, the prominent errors in accelerometer such as scale factor error, bias drift, mechanical vibrations, setup misalignment, cross axis sensitivity, material defects and environment conditions have been shown to affect the performance of accelerometer sensor. Researchers have employed many different types of accelerometer testing procedures depending upon the application area. Therefore, it is important to understand variety of procedures that has been used and their respective impact on overall performance of accelerometer sensor.

\subsubsection{Bias Drift/Offset Error}

The majority of past researchers have focused on the calibration of accelerometer sensor by testing the sensor on a perfectly horizontal platform with zero ' $\mathrm{g}$ ' acceleration. Bias is defined as the non-zero voltage output when only earth gravity vector, zero $g$ is acting on the acceleration sensor. With a constant zero $g$ acceleration acting on the sensor, also known as static acceleration, the bias should remain constant during the static testing. Conversely in dynamic testing, bias drift is seen during the calibration testing. Bias depends upon many factors such as thermal changes, stresses, and self heating. Liu and pang [1] evaluated accelerometer sensor by manually calibrating sensor thermal-bias drift. The performance of accelerometer was tested by making horizontal forward and backward movement with the help of Sony robotic arm. A similar type of research was performed by S. Nikhbakt [24] to compensate bias drift errors in accelerometer sensor using statistical techniques. The performance was tested by moving the sensor platform horizontally to check the position errors. Minha Park and Yang Gao [8] studied error analysis of MEMS, ADXL202JC Accelerometer sensor by evaluating the sensor with 360 degree rotation testing for bias drift errors and scale factor variations. Appropriate error models have been derived by using Auto regressive models. In reference [27] researchers tested RGA300CA system for multi-position testing using a rotary plate. Carver and Looney [28] discussed bias drift calibration depending upon needed performance level. 
Sensitivity analysis is done for $1 \%$ bias correction goal. Acar and Cenk [29] studied the complete analysis of commercially available capacitive type accelerometers based on resolution, frequency response, nonlinearity and transverse response. The experiments were performed on low and high frequency shaking machines, transverse sensitivity testing machine and centrifuge testing. Reported error for ADXL210 was $-0.3 \%$ at 100 Hz. It was observed that the error was least among other commercially available sensors. Researchers [30] have reported the bias error observed for inertial sensors with different grades. Table 2.1 shows the comparison between each grade. Reddy[31] discussed different models and evaluation techniques used in determining drift errors in accelerometer and gyroscope. He reported that Kalman filter can be used to compensate random drift errors.

Table 2.1 Accelerometer Bias Error Comparison [30]

\begin{tabular}{|l|l|l|l|l|l|}
\hline \multicolumn{1}{|c|}{ Grade } & \multirow{2}{*}{$\begin{array}{c}\text { Accelerometer bias } \\
\text { Error }(\mathrm{mg})\end{array}$} & \multicolumn{4}{|c|}{$\begin{array}{c}\text { Horizontal position error } \\
\text { (meters) }\end{array}$} \\
\cline { 3 - 6 } & & $1 \mathrm{sec}$ & $10 \mathrm{sec}$ & $1 \mathrm{~min}$ & $1 \mathrm{hr}$. \\
\hline Navigation & 0.025 & $0.13 \mathrm{~mm}$ & $12 \mathrm{~mm}$ & $0.44 \mathrm{~m}$ & $1.6 \mathrm{~km}$ \\
\hline Tactical & 0.3 & $1.5 \mathrm{~mm}$ & $150 \mathrm{~mm}$ & $5.3 \mathrm{~m}$ & $19 \mathrm{~km}$ \\
\hline Industrial & 3.0 & $015 \mathrm{~mm}$ & $1.5 \mathrm{~m}$ & $53 \mathrm{~m}$ & $190 \mathrm{~km}$ \\
\hline Automotive & 125 & $620 \mathrm{~mm}$ & $60 \mathrm{~m}$ & $2.2 \mathrm{~km}$ & $7900 \mathrm{~km}$ \\
\hline
\end{tabular}

\subsubsection{Scale Factor Error}

Scale factor is the change in output voltage depending on change in input acceleration at nominal supply voltage and temperature. It is measured in $\mathrm{mV} / \mathrm{g}$. Scale factor reflects the sensitivity of the acceleration sensor. Scale factor error is observed when there is a linear deviation from the true scale rate. Scale factor error greatly depends on thermal hysteresis and aging. Most of the researchers used IEEE-STD-1293-1998 recommended four-point or six-point tumble test to calculate scale factor errors. Researchers [8] used rotation panel 
connected to SmartMotor for testing ADXL202JC accelerometer. The sensor was placed at the center of the rotating panel and scale factor error of $0.00667 \%$ was observed for X axis and error of -0.03415 was reported for $\mathrm{Y}$ axis.

\subsubsection{Mechanical Vibrations and Initial Misalignment Error}

Mechanical vibrations are reflected in the acceleration measurement. It becomes difficult to differentiate between acceleration caused by vibration and by actual motion. Mechanical vibrations introduce errors in the calibration which can be minimised by installing the setup on rigid, mechanically isolated, vibration resistant structure. Researchers [28] suggested that for performance goal of $1 \%$, initial alignment error must not exceed by $8^{\circ}$. The error at $0^{\circ}$ itself can cause bias in the measurement. The advantage is measuring acceleration at $0^{\circ}$ and $180^{\circ}$ will have an equal and opposite effect on measurement thus, error will not affect bias drift. By aligning rotator wheel perfectly vertical, misalignment errors can be minimized.

\subsubsection{Cross Axis Sensitivity Error}

For multi axis accelerometer sensors, perfect orthogonal alignment between axes is necessary. Due to mechanical imperfections, inaccurate placement on the testing setup, measurement of one axis can influence by other axis resulting in axis misalignment error. Again by using multi position testing, these errors can be minimised. This error can be explained by general formula,

sensitivity $_{\text {crossaxis }}=\frac{\sqrt{S_{y}^{2}+S_{z}^{2}}}{S_{x}} \times 100$

H. Seidel et.al [32] reported maximum error of $2.5 \%$ for $\mathrm{z}$ axis and concluded that set up misalignment error also contributed in overall error percentage. $180^{\circ}$ rotation test was performed to see the effect of cross axis on $\mathrm{Z}$ axis of an accelerometer sensor. Research [33] reported that the cross axis misalignment error can cause the change in the hysteresis 
characteristic of acceleration sensor. The experiments were performed on ADXL 210 sensor for evaluating effect of hysteresis, nonlinearity, scale factor and bias. The combined effect of any axis measurement was contributed by bias, scale factor and cross axis misalignment. In addition, for capacitive type of accelerometer sensor, proof mass deflection is transformed using capacitive plate arrangement causing minimum cross axis sensitivity error than pendulous design. In fact, Research [34] revealed that for MEMS capacitive type of design, cross axis sensitivity had minimal effect on the measurement.

\subsubsection{Power Supply Variation}

If the sensor output depends on power supply extra care should be taken in providing regulated power supply. Such characteristic is known as" Ratiometricity". Fluctuations in power supply reflect as a spike in output voltage. The calibration can be done at different power supplies to see the effect on collected fitted data [28].

The above mentioned parameters affect the overall performance of accelerometer sensor. Many Researchers considered bias drift and scale factor as prominent error contributors for accelerometer sensor.

\subsection{Acceleration Error Modeling}

Sensor error model can be used at different application stages. These models have proved to be helpful in design, control, calibration and error compensation in real time navigation. The complete error model for pendulous type accelerometer sensor is explained by IEEE-1293-1998 as follows;

$$
\begin{aligned}
& E / K_{1}=K_{0}+\frac{K_{0}^{\prime}}{2} \operatorname{sign}\left(a_{i}\right)+\left(1+\frac{K_{1}^{\prime}}{2} \operatorname{sign}\left(a_{i}\right)\right) a_{i}+K_{o q} a_{i}\left|a_{i}\right|+K_{2} a_{i}^{2}+K_{3} a_{i}^{3}+\sum_{n \geq 4} K_{n} a_{i}^{n} \\
& +\partial_{0} a_{p}-\partial_{p} a_{0}+K_{i p} a_{i} a_{p}+K_{i o} a_{i} a_{o}+K_{p o} a_{p} a_{o}+K_{p p} a_{p}^{2}+K_{o o} a_{o}^{2}+K_{s p i n} w_{i} w_{p} \\
& +K_{\text {ang.accel }} \dot{w}_{0}+\varepsilon
\end{aligned}
$$


Where,

$E / K_{1}=$ accelerometer voltage output divided by scale factor $K_{1}$

$a_{i}, a_{p}, a_{o}=$ applied accelerations along Input Axis (IA), Pendulous Axis (PA) and output axis (OA)

$K_{0}=$ bias, expressed in $\mathrm{g}$

$K_{0}^{\prime}=$ bias asymmetry in $\mathrm{g}$,

$K_{2}=$ nonlinearity, expressed in $\mathrm{g} / \mathrm{g}^{2}$

$K_{3}=$ nonlinearity expressed in $\mathrm{g} / \mathrm{g}^{3}$

$\partial_{o}, \partial_{p}=$ IA misalignment with respect to the OA and PA

$K_{i p}, K_{i o}=$ cross coupling coefficients, expressed in $\mathrm{g} / \mathrm{g}^{2}$

$K_{p p}, K_{o o}=$ cross coupling nonlinearity coefficients expressed as g/cross- ${ }^{2}$

$K_{\text {spin }}=$ as spin correction factor expressed as $\mathrm{g} /(\mathrm{rad} / \mathrm{s})^{2}$

$K_{\text {ang.accel }}=$ is angular acceleration coefficient expressed in $\mathrm{g} /\left(\mathrm{rad} / \mathrm{s}^{2}\right)$

$w_{i} w_{p} w_{o}=$ angular velocity components along IA,PA and OA expressed in $\mathrm{rad} / \mathrm{s}$

$\dot{w}_{i} \dot{w}_{p} \dot{w}_{o}=$ angular acceleration components along IA,PA and OA expressed in $\mathrm{rad} / \mathrm{s}^{2}$.

ADXL335 sensor uses same assembly for mounting $\mathrm{X}, \mathrm{Y}$ and $\mathrm{Z}$ axes, the sensing direction are highly orthogonal [35]. Thus, to build an error model for tri axial MEMS accelerometer sensor, the effect of cross coupling can be ignored [8] [34]. For nonpendulous design, respective error terms would be insignificant [8]. The major error contributor terms were reported by previous researchers are bias drift and scale factor errors. Thus reduced order error equation for single axis accelerometer sensor can be given as;

$E=K_{1}+K_{1} A_{x}+K_{0}+\varepsilon$

Where, 
$A_{x}$ is x axis acceleration measurement. $K_{1}$ and $K_{0}$ as defined in equation 2.1.

Depending upon the bias characteristics, an error model is fitted and tested for residuals [14]. The bias error and scale factor error can be modelled as deterministic input to the Kalman filter to minimize effect of both.

\subsection{Previous Work}

Previous research has considered number of factors that affects performance of accelerometer sensor. Different calibration techniques have been used to overcome with inbuilt sensor errors. Liu and pang [1] evaluated accelerometer sensor for position estimation by double integration of sensor output. Acceleration bias drift was measured by moving robotic arm back and forth at a distance of $40 \mathrm{~cm}$. The measured drift was manually calibrated. Kalman filter was used to combine and process the acceleration data. Joshua Ian and Christian [2] used accelerometer along with low cost potentiometer to estimate automobile hand wheel position and corresponding velocity. Instead of using conventional steering system, they used steer by wire concept that required force feedback. Hand wheel position, velocity, acceleration states were considered for giving this force feedback and corresponding inertia and damping. The results were tested in stationary state as well as moving state of the vehicle. Bouten et.al [36] evaluated triaxial accelerometer for assessment of daily physical activity. In order to imitate the human movements, a laboratory set up was build and tested. The rotary motion was generated with the help of electric motor of lathe. The output was processed with bridge amplifiers and analysed by running ANOVA and Schiff F tests. Reference [14] suggested an error compensation technique for gyro drift errors. The bias drift error model was incorporated in Kalman filter algorithm to estimate the states. The results compared with uncompensated gyro measurements which show drastic improvement with error compensation. The sufficiency of error model was examined by running fitness test for evaluation of orientation estimation. The smoothing algorithm was also developed to increase accuracy of Kalman filter algorithm. Stakkeland et.al [37] tested accelerometer 
performance based on modeling. They built linear acceleration model with acceleration as control input. A separate nonlinear model called continuous Wiener-process acceleration model was also studied. They concluded that the acceleration modeled as a control input give better results than the wiener process model, especially on the vibration and noise related errors. Reference [6] velocity estimation was accomplished using acceleration measurement as input to kinematic Kalman filter (KKF). A comparative study of velocity estimation based on models and KKF was studied. Reference [38] described Position of the prism estimated on a moving base and Kalman filter was used for processing the geodetic movements. Furthermore, Electronic tachometer was used to compare the results. Reference [39] presented a research on an inertial positioning system for a parallel kinematic machine. Experiments were performed with a single parallel kinematic machine test bed. Kalman filter technique was used for Stewart platform PKM application. Thus, inertial sensor based PKM-TCP positioning system was established to measure the pose of TCP. An interesting application of Kalman filter is described in model based tracking of moving object [3] using camera and a servo device. Furthermore, model matching was accomplished using Kalman filter technique. J Gao, P Webb and N Gindy [40] proposed error analysis technique to calibrate inertial sensors for machine tool applications. They reported position error of $0.07 \mathrm{~mm}$ in one axis strut movement. It was also concluded that vibrational motion captured by inertial system was more accurate than encoder system.

Most of the research work accomplished in acceleration error analysis have used horizontal platform for evaluating performance of calibrated sensor with different data processing algorithms and techniques. Furthermore, sensors were mounted on mobile platform rather than on the wheel itself for investigating the acceleration measurement. There is no standard method to incorporate error model in a system for calibration of these sensors. IEEE Gyro-Acceleration panel is currently preparing document P1554 for recommended practice of inertial sensor test equipment, instrumentation, data acquisition system and analysis. The limited results on accelerometer error compensation in inertial 
sensor fusion suggest that in order to determine position under various conditions, experimental testing and calibration of inertial sensors should be performed. In our approach, ADXL 335 by Analog Devices [35] was mounted on rotary wheel at different rotational speed to calibrate and evaluate the sensor. Fitted data is used to incorporate bias drift error as a deterministic input to Kalman filter for short distance position estimation in absence of external reference sensor. 


\section{CHAPTER 3}

\section{Testing Program, Methods and Procedures}

This chapter is divided into two sections. The first section describes ADXL335 accelerometer IC used in this study and its principle, properties and sensor mounting technique used in this program. The second section details the testing procedure used to evaluate the error sources, mechanical design components and NiDAQ USB 6009 data acquisition card functionality.

\subsection{Evaluation of MEMS Accelerometer Sensor}

With modern nanotechnology, it is now possible to bring sensors, their signal conditioning and processing circuits on a single silicon based platform. The technology used for this purpose is called as micro electro mechanical (MEMS) system [41]. Due to its small size and ease of operation, MEMS accelerometer sensor is commonly used in gaming applications to enhance the effect, mobile handsets, even implemented on human body to analyse tremors and other physical activities.

\subsubsection{Principle of MEMS Accelerometer Sensors}

Figure 3.1 shows basic principle of accelerometer sensor. The sensor is made of spring loaded, micro machined structure, mounted on silicon base. Force on the structure changes the position of seismic mass attached on the spring. This deflection is measured using fixed plate capacitor sensors. The change in acceleration unbalances capacitor plate distance, observed by modulation/demodulation circuits and thus, resulted in output proportional to acceleration. The sensing can be static (gravity) or dynamic (forced acceleration) [42]. 


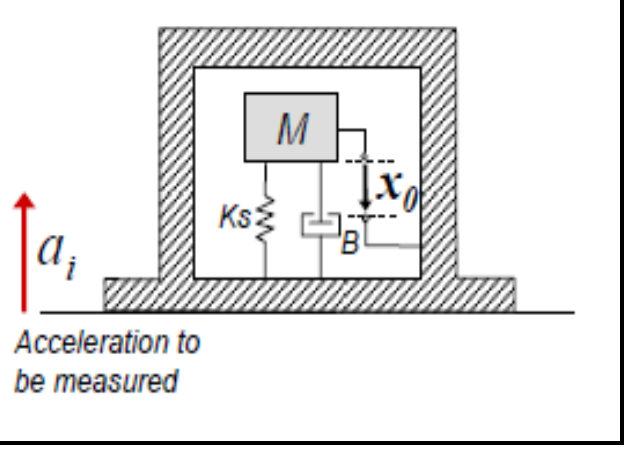

Figure 3.1 Accelerometer Sensing Principle

\subsubsection{Acceleration Sensor Mounting}

The two acceleration sensors were mounted on a vertical rotating panel for testing the sensor. The distance between center of the shaft and acceleration sensor is set at $\mathrm{R}=0.1 \mathrm{~m}$. When sensors $A_{1}$ and $A_{2}$ are aligned to horizontal axis, the only force acting on the sensor is gravity. Thus expected output is zero. The sensor is then oriented at $90^{\circ}, 180^{\circ}$ and $270^{\circ}$ along the horizontal axis and the acceleration data is recorded for both the axes. $\mathrm{Z}$ axis is perpendicular to the vertical rotation panel as shown in the figure 3.2.

The relation between angle and acceleration is given by,

$$
a_{x}=\sin \left(\theta_{x}\right)
$$

Differentiating,

$$
\frac{d a_{x}}{d \theta_{x}}=\cos \left(\theta_{x}\right)
$$




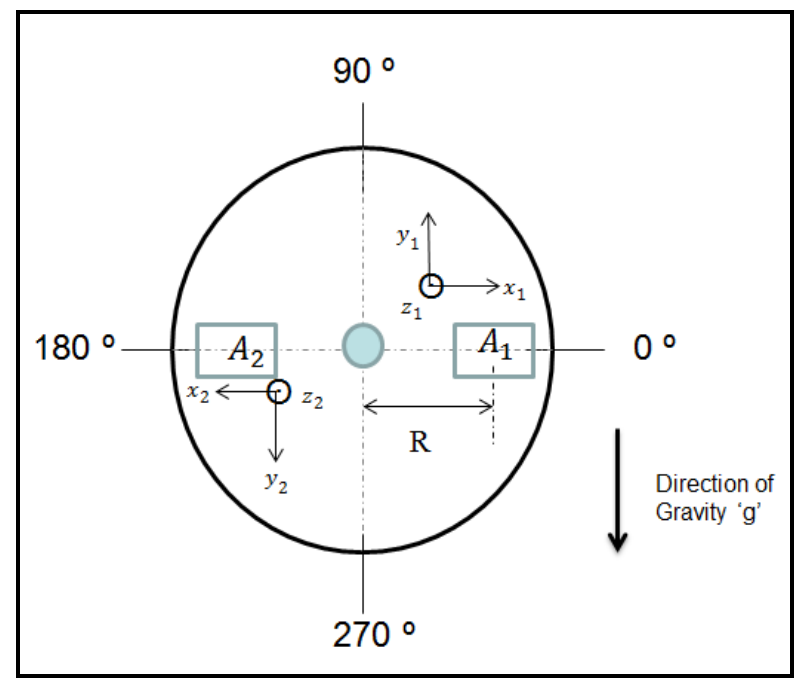

Figure 3.2: Accelerometer Sensors Mounting

Thus at 90 degrees, the acceleration sensitivity decreases to zero. In order to measure complete 360 degree rotation, two axis sensors were chosen. It was observed that the decrease in the sensitivity of one axis causes an increase in the other axis as both the axes are orthogonal to horizon [43].

\subsection{Mechanical System Design}

To test the simulations, a hardware set up was built which consists of a rotating Plywood wheel. Figure 3.3 shows hardware setup block diagram. The acceleration sensors were mounted on the wheel which is driven by Dayton's DC motor. The controller controls the speed of DC motor for testing calibration. ADXL 335 mounted on wheel, gives output voltage proportional to measured acceleration. Spark fun's ADXL 335 breakout board has inbuilt capacitors for filtering the data coming from sensor as a first stage of processing. In turn, National Instrument's USB 6009 data acquisition card converts this data by using successive approximation 16 bit ADC converter. 


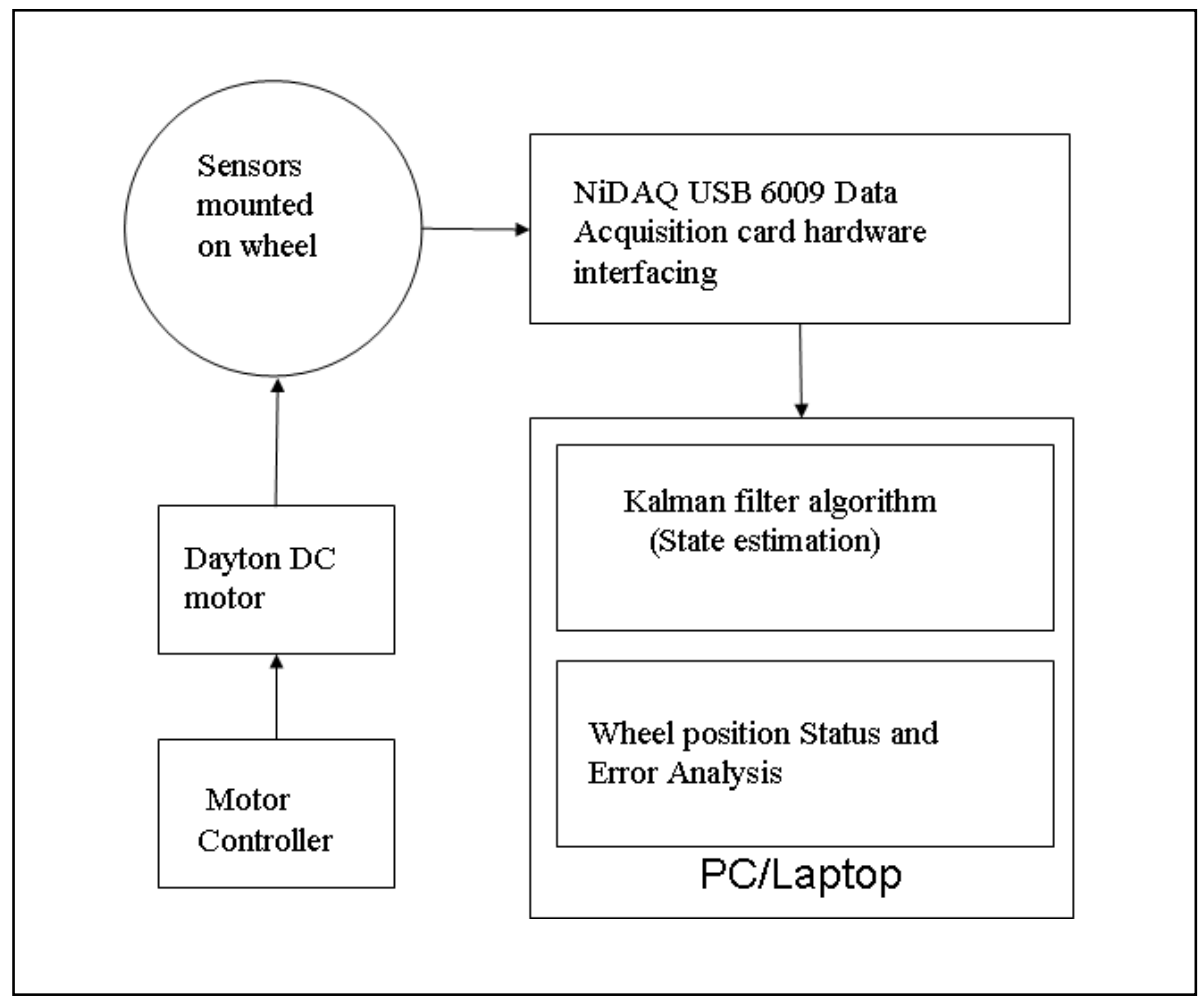

Figure 3.3 Hardware Setup Block Diagram

The interfacing of this data acquisition card to computer is performed by using Measurement and Automation explorer and NiDAQmx device drivers. Figure 3.6 shows the interfacing VI. LabVIEW software is used to log the data on the disk. This raw data is filtered with the help of Kalman filter algorithm. The position status is evaluated based on filtered data. 


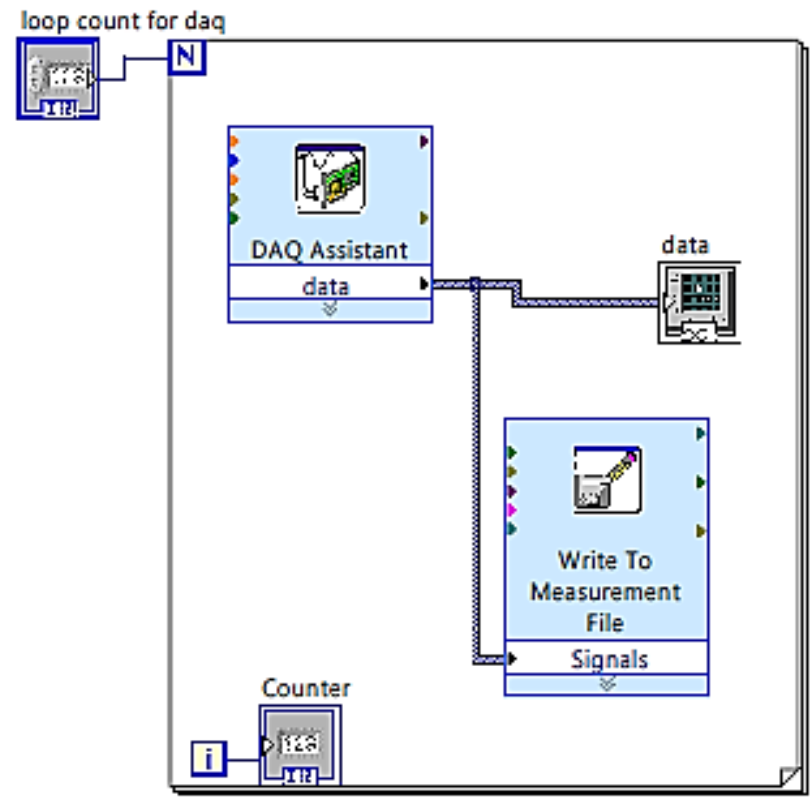

Figure 3.4 NiDAQ USB 6009 LabVIEW Interfacing.

\subsubsection{Accelerometer Evaluation ADXL 335}

A number of factors was considered for acceleration sensor selection. Sensor with small measurement range was selected as resolution degrades with increase in the sensing capability. Additionally, power supply and thermal stability with optimum bias were the important parameters to be considered for the experiment. The accelerometer used for the research is ADXL335 from Analog Devices. ADXL 335 is tri axial accelerometer sensor with built-in amplifiers and demodulator. ADXL335 has a range of $\pm 3 \mathrm{~g}$ in each axis. The output shows ratiometric characteristics thus, output voltage changes depending on the change in supply voltage. Nominally, at zero g, output voltage is half of the supply voltage. Each axis has a separate bandwidth adjustments. The capacitor in series with output decides the bandwidth of each axis. The accelerometer can be used for measuring dynamic acceleration such as vibration as well as static motion e.g. gravity. Bandwidth range of $200 \mathrm{~Hz}$ to $1 \mathrm{KHz}$ is used depending on the rotational speed. This research used two, three axis accelerometer sensors as illustrated in figure 3.5. The analog voltage 
output from three different axes is fed to NiDAQ USB 6009 for sampling and signal conditioning.

Figure 3.5 shows basic block diagram of ADXL 335 as mentioned in the data sheet. The chip has in-built coupling capacitor between supply voltage and ground; thus reducing the noise at the power supply line. It contains micro machined three axis sensor along with signal conditioning circuitry.

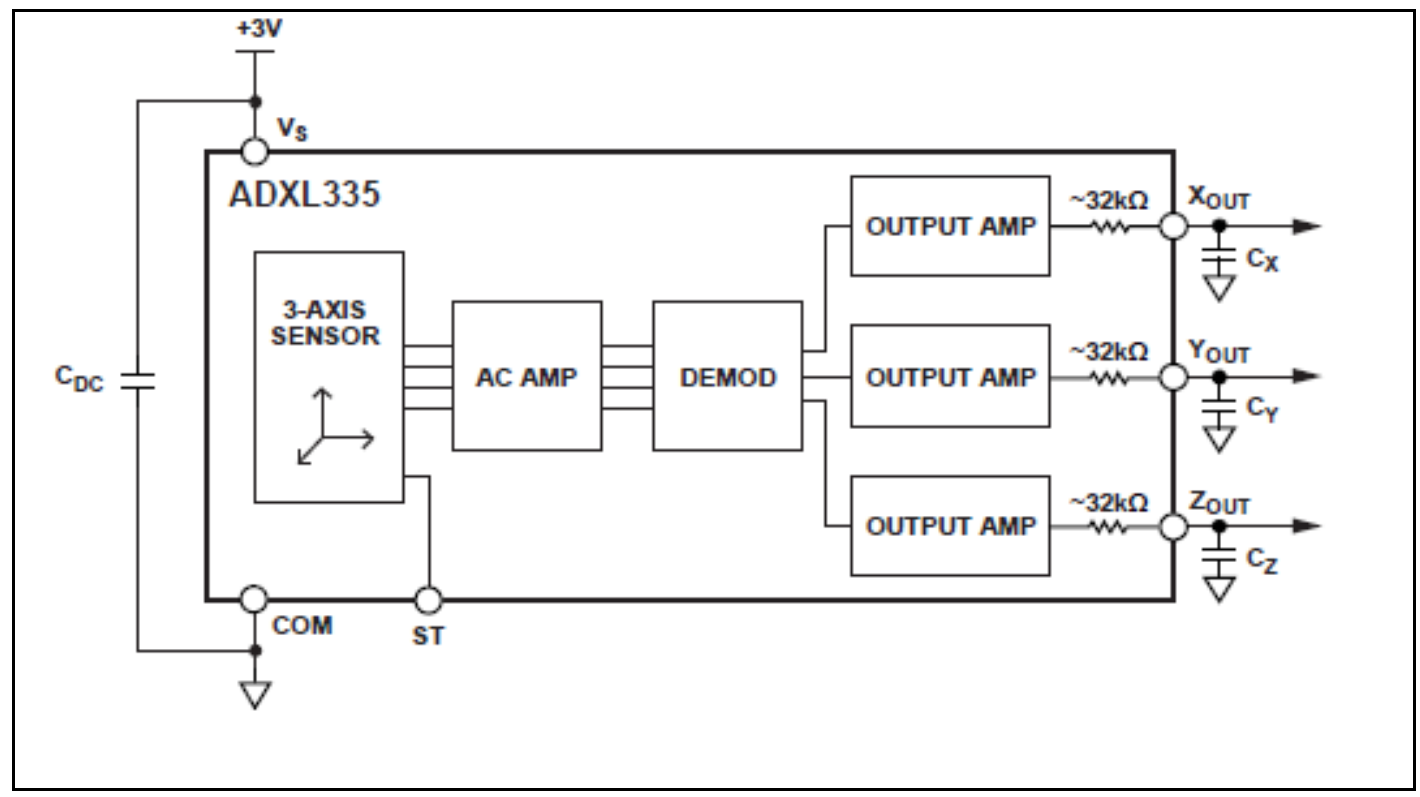

Figure 3.5 ADXL 335 block diagram [35]

The sensor output data is acquired using LabVIEW and DAQ card and processing is completed with MATLAB called through LabVIEW for optimum filtering. The capacitor value is selected as $0.1 \mu \mathrm{F}$ for sensor frequency of $50 \mathrm{~Hz}$.

\subsubsection{NiDAQ - USB 6009}

N Series data acquisition card NiDAQ USB-6009 by National Instruments is used to acquire voltage output signals. The data card provides support with LabVIEW for device 
configuration. Figure 3.5 shows basic electronics used in National Instrument's USB 6009 data acquisition card. The card consists of eight analog inputs and two analog outputs in addition to 12 digital I/O lines. Furthermore, it has 14 bit ADC resolution with a sampling rate of $48 \mathrm{KHz}$ per channel.

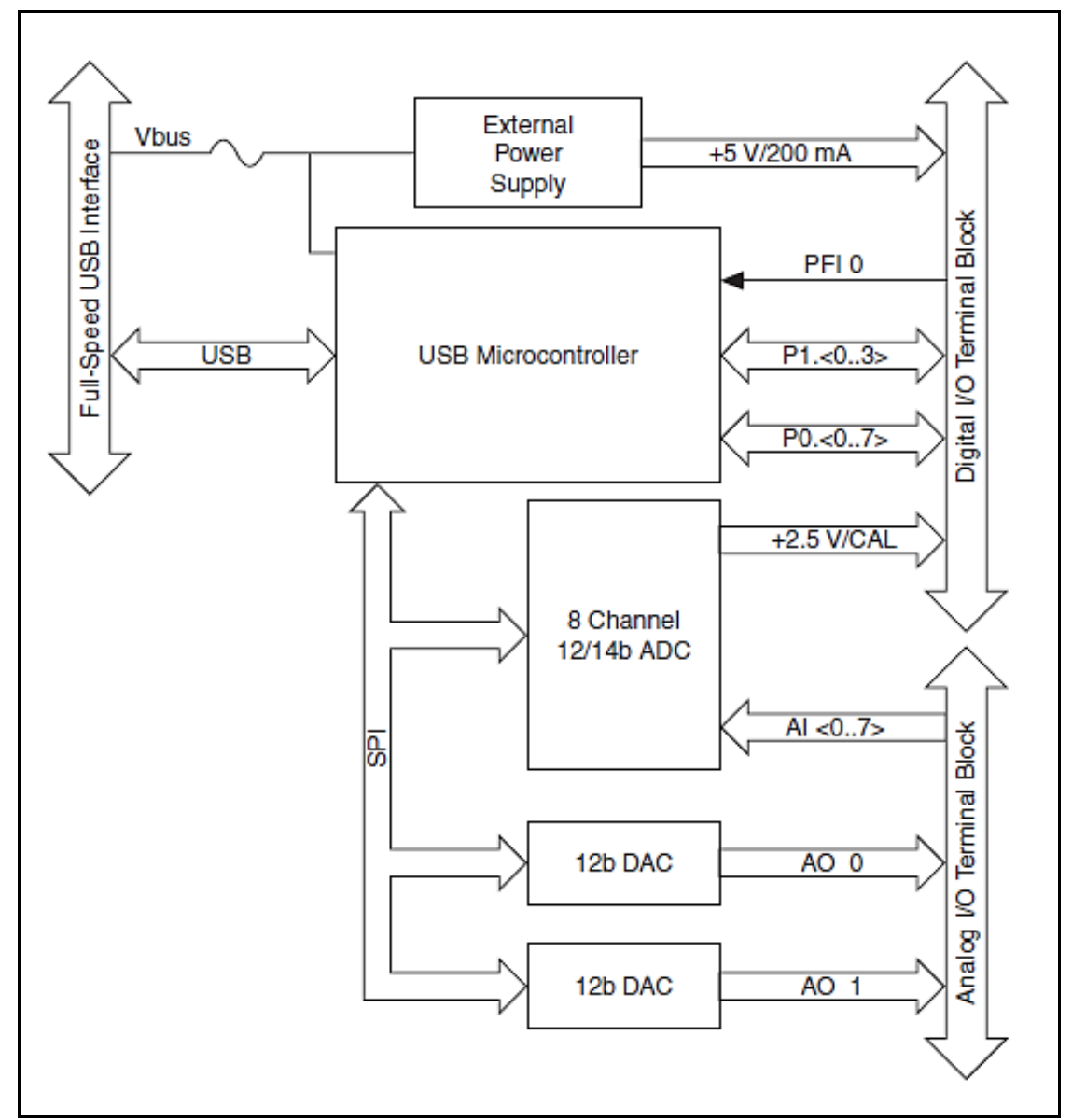

Figure 3.6 National Instrument’s USB 6009 block diagram

The device comes with NiDAQmx drivers which are compatible with LabVIEW and windows for processing. Serial communication on PC is established through USB interface. 
LabVIEW is good for Data acquisition with user friendly interfacing. MATLAB has inbuilt mathematical functions for better signal processing. The figure 3.7 shows execution of MATLAB program from LabVIEW to get benefit of both softwares.

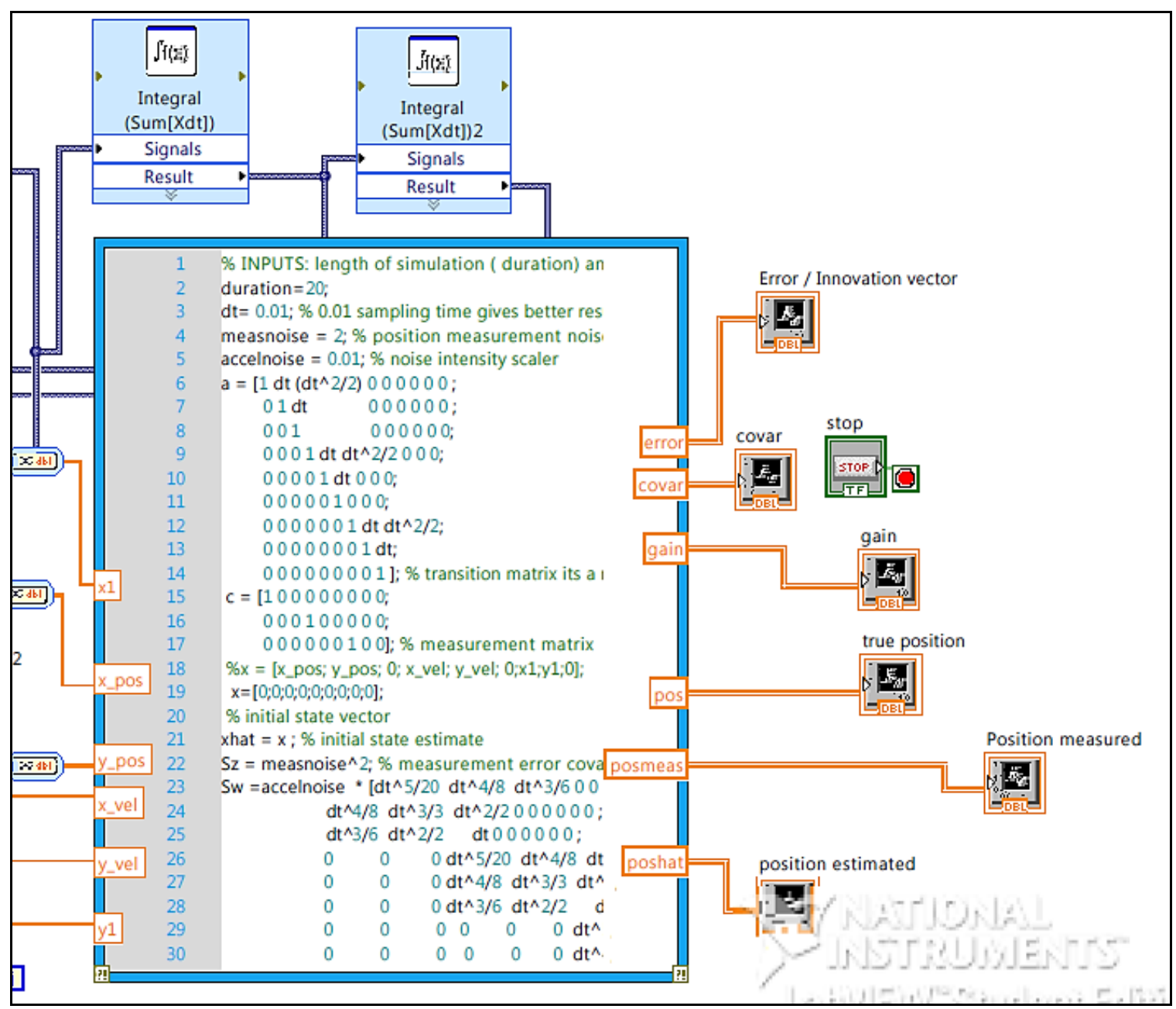

Figure 3.7 MATLAB Functions Call from LabVIEW 


\subsubsection{Rotating Wheel Arrangement}

The wheel attached to DC motor shaft simulates the rotary motion. The orthogonality can be tested between the base of motor and vertical shaft to ensure perfect vertical position of the wheel. The system provides fairly accurate servo table system. The arrangement is maintained in a lab with moderately constant temperature to ensure minimum thermal bias drift. The data obtained from the sensors is directly and accurately fed into laptop for further processing. The tests are repeated several times to find the residuals. The test setup consists of rigid mounting circular plate with acceleration sensors attached at the radius of $0.1 \mathrm{~m}$ from the center shaft. The setup is tested at various constant speeds. The system is as shown in the figure 3.8 .

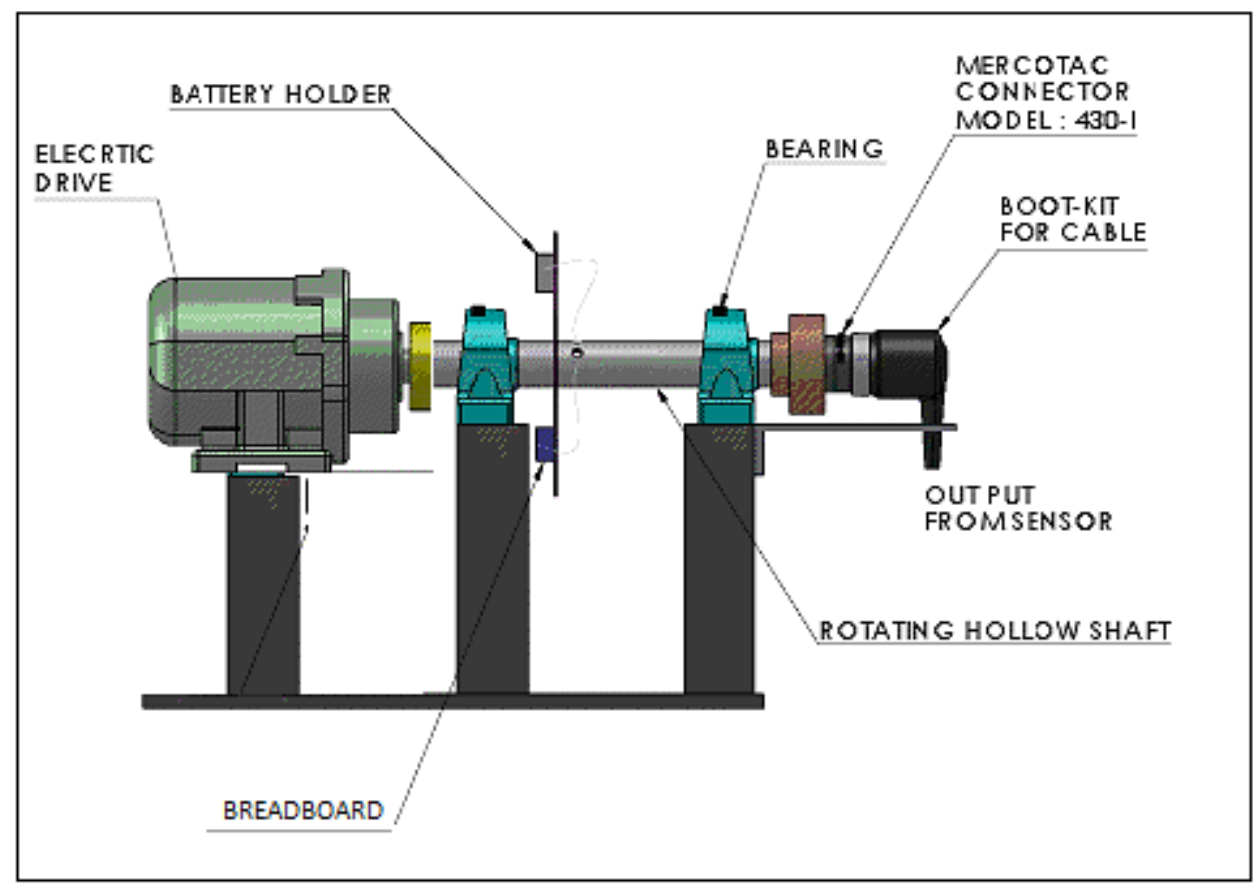

Figure 3.8 Hardware Test Set Up

The circular motion is transferred from DC motor to rotating hollow shaft. Coupling is used to join Dayton DC motor to shaft. The sensors mounted on plywood wheel and 
output wires taken inside the shaft and through slip ring to the stationary end for connection with NiDAQ USB card.

\subsubsection{Slip Ring Arrangement}

Sensory data is transferred to NiDAQ device through serial connection. The rotary motion causes wires to get twisted around the shaft. The electromechanical connector such as slip ring is used to run the data and power lines through the shaft to minimize twisting.

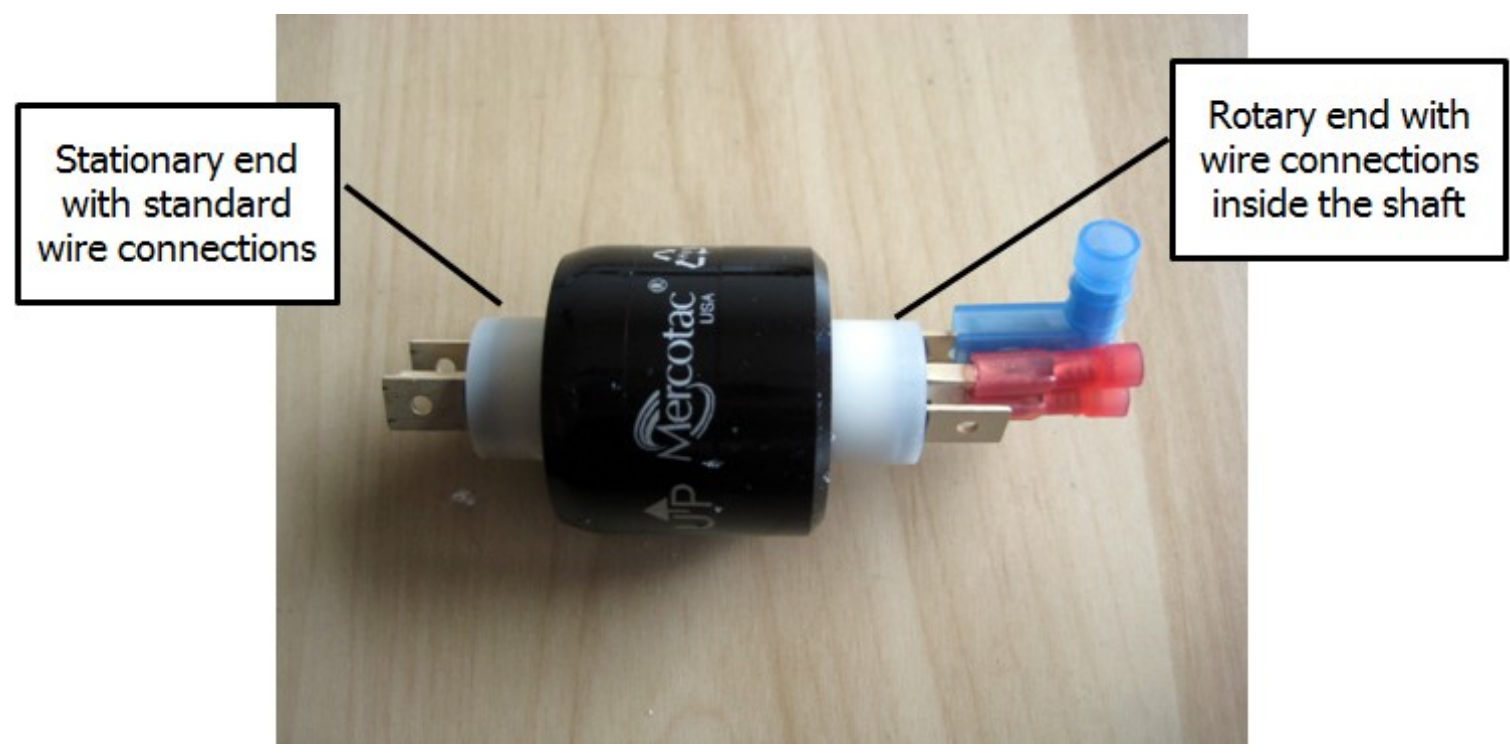

Figure 3.9 Mercotac Inc.'s Electrical Connector [44]

Slip ring is used to transfer data and power lines from the sensors mounted on rotary platform to data acquisition unit. For the evaluation of accelerometer sensor it is very necessary to extract the noise. Traditional brush type slip ring assembly would cause considerable resistance in the measurement. Therefore, Liquid metal rotating electrical connector from "Mercotac" [45] is used instead of traditional brush type slip ring to avoid transmission noise. The new electrical connector is used liquid mercury instead of brush type arrangement which greatly reduces resistance through the rotating contact points. 
Additionally, durable and maintenance free continuous operation was an advantage for selecting serial communication using connectors over the wireless communication.

\subsection{Four Point Tumble Test}

For evaluating bias error characteristics, the sensors were mounted on rotating wheel that is firmly attached to a rotating shaft with fixed supports. Testing wheel was carefully levelled with gravity vector so that $\mathrm{x}$ and $\mathrm{y}$ axis are orthogonal to gravity. For static testing, data was reported by titling accelerometer position at various angles. As described in IEEE-1293-1998 standard, the four point tumble test is performed to calibrate the sensor on a vertical platform to see the bias error effect in rotation. The sensor placement is as shown in the figure 3.10. Error parameters were estimated with lab testing of wheel with known rotation angles. The experiment was performed for a number of consecutive rotations and repeated for several times to observe the error fluctuations. The bias drift and sensitivity analysis was performed by calculating the mean at $0^{\circ}$ and $180^{\circ}$ degree for ideal measurement and real time data.

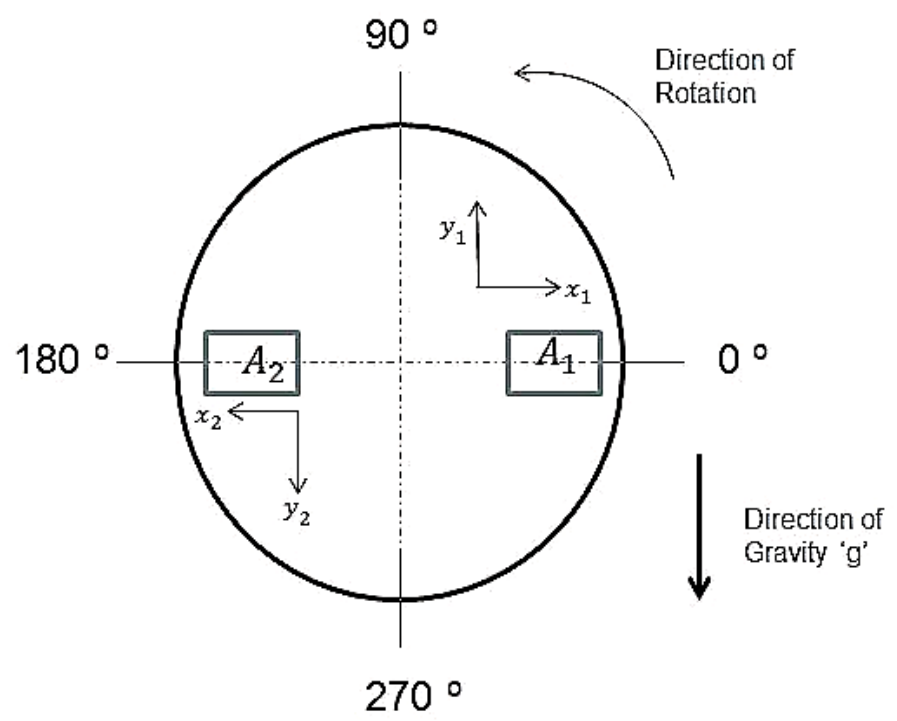

Figure 3.10 Four-Point Tumble Test Illustration 


\section{CHAPTER 4}

\section{Software System Design}

In this chapter, brief introduction to basic Kalman filter is provided and implementation for position estimation is presented. The algorithm was developed for calculating acceleration bias error and scale factor error using acceleration error model [12][46]. Additional algorithm was developed for data acquisition and processing of three axis accelerometer using Kalman filter techniques.

\subsection{Theory and Implementation of Kalman filter}

The Kalman filtering method for noise reduction was first proposed by R.E Kalman as an alternate solution to Wiener's minimum mean squared error filtering method. As this filter worked in frequency domain, storing the entire measurement data was required. The advantage of Kalman filter over wiener is its recursive property thus, very popular in applications such as inertial navigation system, missile control and marine applications. Kalman filtering algorithm is developed from least square estimation technique, which is used in determining states of vector, time varying signals. The function of Kalman filter is explained in depth in references [11][47].

Kalman filter is a recursive algorithm for data processing primarily for linear systems. Dynamic system's state can be estimated by Kalman filtering. Kalman filter takes noisy dynamic measurements as input and estimate the states.

It consists of the following;

a) Process model

b) Measurement model 


\section{a) Process model}

It uses knowledge of the system, sensor dynamics such as error covariance and initial condition, in our case, initial position, velocity and acceleration. General linear stochastic equation can be represented as,

$x_{k}=A x_{k-1}+B u_{k-1}+w_{k-1}$ Where, $x \in R^{n}$

Kalman filter solves the state estimation problem of a discrete time process [11].The subscript $k-1$ is the time step when measurement y is not considered for estimation. $k$ is the predicted time step using measurement $\mathrm{y} . x_{k}$ is state of the system with matrix dimensions $n \times 1$ where $n$ represents number of states. A posteriori state $\hat{x}_{k}^{+}$depends upon a priori state, $x_{k}^{-}$and the process noise $w_{k} . A$ is state transition matrix contains equation of the system in matrix form. $u_{k}$ represents known control input also termed as deterministic input which is related by matrix $B(n \times 1)$.

State transition matrix $n \times n$ transforms the state $x_{k}$ from time index $k-1$ to $k$. The process noise $w_{k}(n \times 1)$ is assumed to be Gaussian distributed white noise and the covariance or deviation in process noise is denoted by Q.

$E\left[w_{k} w_{j}^{T}\right]=Q_{k}$ When, $j=k$

$E\left[w_{k} w_{j}^{T}\right]=0 \quad$ When, $j \neq k$

E is the expected value of $w_{k}$ when multiply by its transpose $w_{j}^{T}$.

b) Measurement Model

The measurement model is given by,

$$
y_{k}=C x_{k}+v_{k}
$$


$y_{k}$ represents actual measurement $(m \times 1)$ which is a function of $x_{k}$. Kalman filter uses available measured value to estimate the state of the system $x_{k}$ which is corrupted by measurement noise, $v_{k}(m \times 1)$ with covariance $\mathrm{R}$.

$E\left[v_{k} v_{j}^{T}\right]=R_{k}$ When, $j=k$

$E\left[v_{k} v_{j}^{T}\right]=0$ When, $j \neq k$

E is the expectation of $v_{k}$ when multiply by its transpose $v_{j}^{T}$.

We also assume that $v_{k}$ and $w_{k}$ have no correlation expressed in mathematical term as,

$E\left[w_{k} v_{j}^{T}\right]=0, \forall k, j$

Thus vectors $v_{k}$ and $w_{k}$ are independent random variables.

Matrix $\mathrm{C}$ is called as observation matrix which relates to the state of system to actual measurement with dimensions $m \times n$.

\subsubsection{Kalman Filter Algorithm}

The algorithm can be divided into two parts

a) Prediction stage

b) Correction stage

a) Prediction stage uses the initial conditions and system model to predict the future state. The prediction state consists of process model;

$$
\hat{x}_{k}^{-}=A \hat{x}_{k-1}^{+}+B u_{k-1}
$$

$A$ is state transition matrix contains the equations of the system in matrix form.

$\hat{x}_{k}^{-}$is a priori state with minus sign and hat indicates it's the estimate before including actual measurements at time index $\mathrm{k}$. the error between a priori estimate and the actual state is given by [48],

$e_{k}^{-}=x_{k}-\hat{x}_{k}$ 
the estimated error covariance (mean squared error) associated with $\hat{x}_{k}^{-}$[48] is given by equation,

$P_{k}^{-}=E\left[e_{k}^{-} e_{k}^{-T}\right]=E\left[\left(x_{k}-\hat{x}_{k}^{-}\right)\left(x_{k}-\hat{x}_{k}^{-}\right)^{T}\right]$

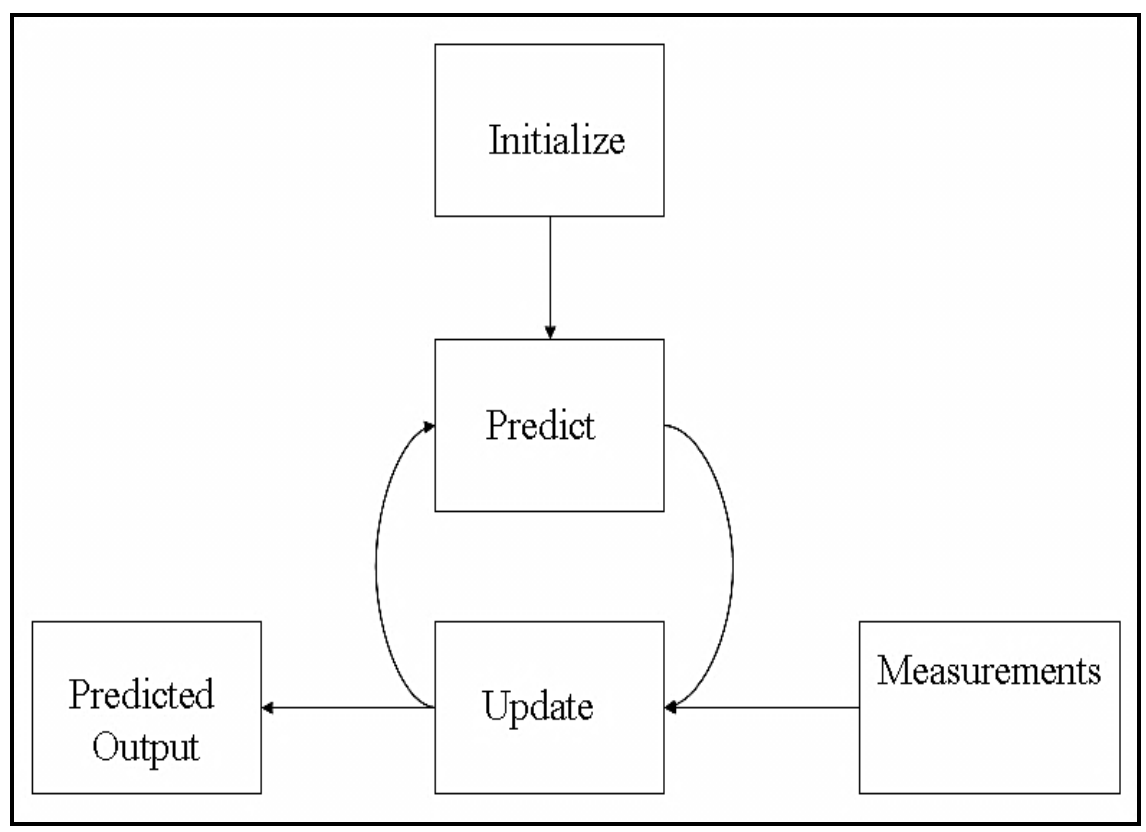

Figure 4.1 Kalman Filter Block Diagram

Error covariance matrix is calculated by,

$\bar{P}_{k}=A P_{k-1}^{+} A^{T}+Q$

$\mathrm{P}$, error covariance matrix, predicts a priori state $\hat{x}_{k}$ by using state transition matrix A. Covariance matrix represents correlation between states $\hat{x}_{k}$.From equation (4.5) and (4.7) it is observed that, prediction is made after adding process noise covariance error in the measurement. Thus, the error was added in the prediction.

b) To correct the prediction according to current measurement, Correction stage is added as a feedback as shown in figure 4.1. It consists of three equations. Each contributes to correct the estimation depending upon the actual measurement and gain. 
A new measurement $y_{k}$ is introduced to a priori state $\hat{x}_{k}^{-}$to get better a posteriori state estimate $\hat{x}_{k}$ and a posteriori estimate $P_{k}$ using equation,

$$
\hat{x}_{k}^{+}=\hat{x}_{k}^{-}+K_{k}\left(y_{k}-C \hat{x}_{k}^{-}\right)
$$

Where, $\hat{x}_{k}^{+}$is a posteriori state estimate also known as corrected state. The term , $\left(y_{k}-C \hat{x}_{k}^{-}\right)$is called as residual error or innovation vector and $K_{k}$ is a multiplying factor also called as Kalman gain, Kalman gain decides if the measurement is trustworthy or not.

Kalman gain is calculated by the equation,

$$
K_{k}=A P_{k}^{-} C^{T}\left(C P_{k}^{-} C^{T}+R\right)^{-1}
$$

Kalman gain value decides error covariance matrix $P_{k}$ by equation,

$$
P_{k}=\left(I-K_{k} C\right) P_{k}^{-}
$$

Depending upon the occurrence of correcting the estimation, process covariance reduces and stabilises at a minimum value where there is a little need to correct the predictions further. This is called as filter convergence.

\subsubsection{Kalman Gain}

From equation (4.9) it can be concluded that Kalman gain is inversely proportional to measurement noise covariance R. With less noise in the measurement, gain value increases resulted in more confidence in actual measurement than the prediction. Kalman gain is based on matrices $\mathrm{A}, P_{k}$ and $\mathrm{R}$ thus, the calculation was performed offline for linear systems to save computational burden. 
When the current measurement is not trustworthy, $P_{k}^{-}$value tends to become zero and the filter takes the predicted value over noise measurements which can be described as,

$$
K_{k}=0, \hat{x}_{k}=\hat{x}_{k-1}
$$

And conversely, when the measurement is within the expectable range, matrix $R$ tends to zero and the prediction is neglected as explained by the equation,

$K_{k}=H^{-1}, \hat{x}_{k}=H^{-1} y_{k}$

The whole process can be summarized as shown in figure 4.2.

\subsubsection{Kalman Filter Extension}

The previous Kalman filter equations are valid for linear systems. For highly nonlinear measurements there is a need to focus on nonlinear Kalman filtering extensions such as extended Kalman filter, and particle Kalman filter. For nonlinear applications initial guess of trajectory is unpredictable and most of the cases it is unknown thus, Kalman filter's a priori estimate is taken as nominal trajectory for prediction. Filter assumes that the probability distribution function is gaussian and has an approximate linear relationship with input and output thus the covariance matrix changes as time change. Extended Kalman filter linearizes nonlinear functions with the help of first order Taylor series expansion [49]. 


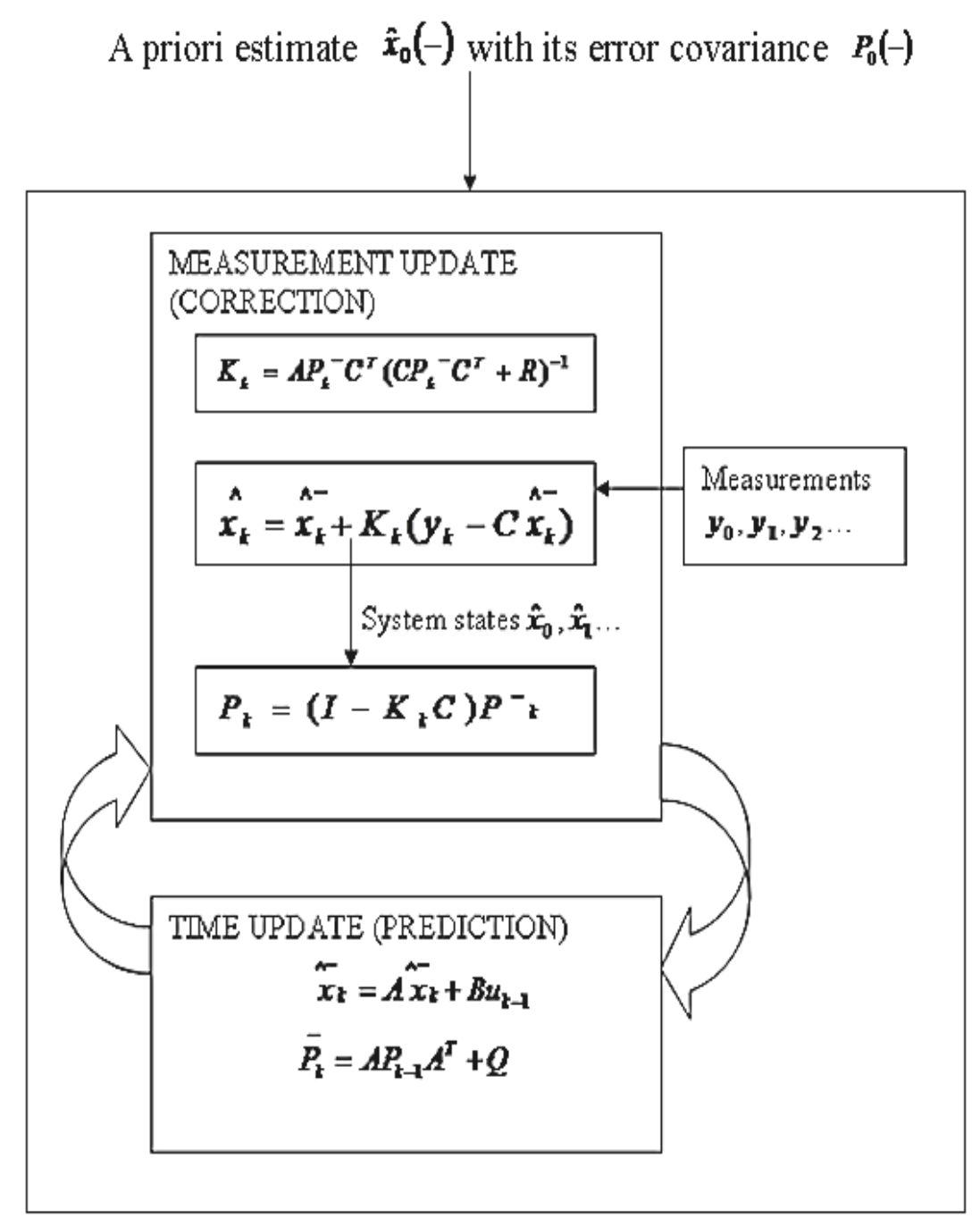

Figure 4.2 Kalman Filter Algorithm

The linearization is performed by representing states as deviation from mean value of Taylor approximation. The a priori state $\hat{x}_{k}^{-}$is used to set up predetermined trajectory. In extended Kalman filter process model and observation model can be described as follows;

$$
\begin{aligned}
& x_{k}=f\left(x_{k-1}, u_{k-1}, w_{k-1}\right) \\
& y_{k}=h\left(x_{k}, v_{k}\right)
\end{aligned}
$$


Where $w_{k}$ and $v_{k}$ are process and measurement noises with Covariances $\mathrm{Q}$ and $\mathrm{R}$ respectively which represents Gaussian white noise characteristics. Taylor series expansion of the state equation 4.13 around $\hat{x}_{k+1}^{+}$is given by [49]

$x_{k}=f_{k-1}\left(\hat{x}_{k-1}^{+}, u_{k-1}, 0\right)+\frac{\partial f_{k-1}}{\partial x}\left|\hat{x}_{k-1}^{+}\left(x_{k-1}-\hat{x}_{k-1}^{+}\right)+\frac{\partial f_{k-1}}{\partial w}\right| \hat{x}_{k+1}^{+}\left(w_{k-1}\right)$

After solving above equation we get,

$x_{k}=F_{k-1} x_{k-1}+\widetilde{u}_{k-1}+\widetilde{w}_{k-1}$

The deterministic signal $\widetilde{u}_{k}$ and process noise $\widetilde{w}_{k}$ is given by

$\widetilde{u}_{k}=f_{k}\left(\hat{x}_{k}^{+}, u_{k}, 0\right)-F_{k} \hat{x}_{k}^{+}$

$\widetilde{w}_{k} \approx\left(0, L_{k} Q_{k} L_{k}^{T}\right)$

In extended version of Kalman filtering, transition matrix and the observation matrix changes with respect to time about the measurement instant, $\hat{x}_{k-1}^{+}$. Thus,

For $\mathrm{k}=1,2 \ldots$

$$
\begin{aligned}
& F_{k}=\frac{\partial f_{k}}{\partial x} \mid \hat{x}_{k-1}^{+} \\
& L_{k}=\frac{\partial f_{k}}{\partial w} \mid \hat{x}_{k-1}^{+}
\end{aligned}
$$

Thus, states and measurement equations can be stated as;

$x_{k}=f_{k-1}\left(x_{k-1}, u_{k-1}, w_{k-1}\right)$

$y_{k}=h_{k}\left(x_{k}, v_{k}\right)$

Where,

$w_{k} \approx\left(0, Q_{k}\right)$

$v_{k} \approx\left(0, R_{k}\right)$

For extended Kalman filter state transition matrix varies as time updates.

The time update equations states for EKF are given by; 


$$
\begin{aligned}
& P_{k}^{-}=F_{k-1} P_{k-1}^{+} F_{k-1}^{T}+L_{k-1} Q_{k-1} L_{k-1}^{T} \\
& \hat{x}_{k}^{-}=f_{k-1}\left(\hat{x}_{k-1}^{+}, u_{k-1}, 0\right)
\end{aligned}
$$

With the partial derivative matrices around $\hat{x}_{k}^{-}$as,

$$
\begin{aligned}
& H_{k}=\frac{\partial h_{k}}{\partial x} \mid \hat{x}_{k}^{-} \\
& M_{k}=\frac{\partial h_{k}}{\partial v} \mid \hat{x}_{k}^{-}
\end{aligned}
$$

Measurement update equations are given by[49]:

$$
\begin{aligned}
& K_{k}=P_{k}^{-} H_{k}^{T}\left(H_{k} P_{k}^{-} H_{k}^{T}+M_{k} R_{k} M_{k}^{T}\right)^{-1} \\
& \hat{x}_{k}^{+}=\hat{x}_{k}^{-}+K_{k}\left[y_{k}-h_{k}\left(\hat{x}_{k}^{-}, 0\right)\right] \\
& P_{k}^{+}=\left(I-K_{k} H_{k}\right) P_{k}^{-}
\end{aligned}
$$

The main difficulties in implementation of extended Kalman filter is the difference between the predicted state and true state. As the difference increases, filter becomes non stable and fails to linearize the equations. Since transition matrix and covariance matrix changes with time, all the calculation is performed online and thus increases the complexity of the algorithm.

\subsubsection{Noise Covariance Tuning}

Performance of Kalman filter is largely dependent upon noise uncertainty matrices Q and R. Adjusting the diagonal matrix values of $\mathrm{Q}$ to give optimum Kalman solution is called as tuning. These tuning parameters reflect white noise associated with process model errors. Parameter evaluation is done by starting with maximum known error values prediction for the states and the values are later adjusted by observing best possible filtering solution by trial and error. 
For one dimensional PVA model, the Q matrix for one dimensional PVA measurement is given by,

$$
Q=E\left(x_{k} \times x_{k}^{T}\right)
$$

Q matrix is formed by considering variance of all states of the measurement. Off diagonal states represents Covariances as explained by equation,

$$
\begin{aligned}
& Q=E\left[\left\{\begin{array}{l}
\sigma_{p} \\
\sigma_{v} \\
\sigma_{a}
\end{array}\right\} \times\left\{\begin{array}{lll}
\sigma_{p} & \sigma_{v} & \left.\sigma_{a}\right\}
\end{array}\right]\right. \\
& Q=\left[\begin{array}{ccc}
\sigma_{p}^{2} & \sigma_{p} \times \sigma_{v} & \sigma_{p} \times \sigma_{a} \\
\sigma_{v} \times \sigma_{p} & \sigma_{v}^{2} & \sigma_{v} \times \sigma_{a} \\
\sigma_{a} \times \sigma_{p} & \sigma_{a} \times \sigma_{v} & \sigma_{a}^{2}
\end{array}\right]
\end{aligned}
$$

We assume that the noise sources are not correlated, thus, off diagonal elements can be taken as zero. A priori estimation error $P_{k}^{-}$is used to calculate Kalman gain along with the measurement uncertainty R.

$$
K_{k}=A P_{k}^{-} C^{T}\left(C P_{k}^{-1} C^{T}+R\right)^{-1}
$$

By observing the equation (4.27), we know that $\mathrm{Q}$ governs $\mathrm{P}$ which ultimately affects the Kalman gain K. Thus, Kalman gain changes in proportion to the process noise covariance $\mathrm{Q}$, When $\mathrm{Q}$ is large, $\mathrm{P}$ and $\mathrm{K}$ will be large and filter responds to the changes quickly. When Q is small, latency in the filter convergence is seen. Q also depends upon the step size as shown in the figure 4.4. As the step size increases, covariance decreases and settles at the value less than 10 within 20 steps. The initial guess of $\mathrm{P}$ is large which reflect less confidence on the measurement and as time progress, depending upon the $\mathrm{P}$, $\mathrm{Q}$ and gain value, covariance decreases over the time. 


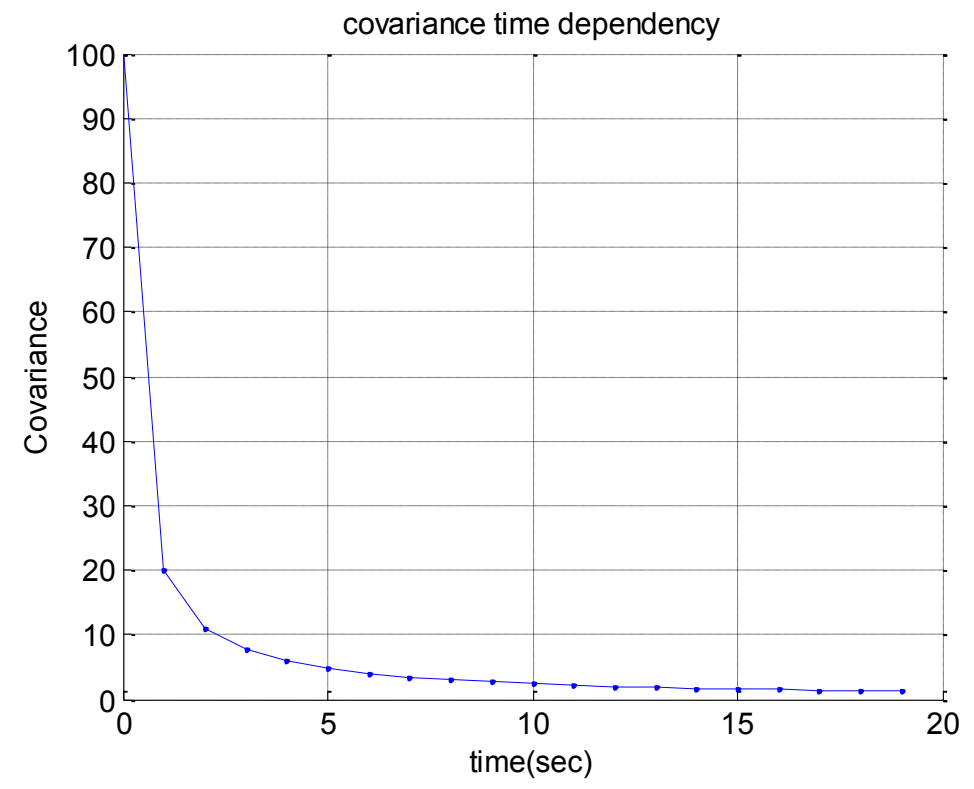

Figure 4.3 Covariance Time Dependencies

It is observed that Kalman gain is inversely proportional to measurement uncertainty R. when $\mathrm{R}$ is small, Kalman gain value increases giving fairly accurate parameter estimation at correction stage. It uses the current observation to predict next estimation.

The accelerometer sensor measurement uncertainty can be modelled as,

$R_{k}=\operatorname{diag}\left[\sigma_{\text {acceleration }}^{2}\right]$

Where $\mathrm{R}$ is the measurement uncertainty associated with measurement model. The measurement uncertainty is usually provided by manufacturer in the data sheet as standard deviation. The tuning of $\mathrm{R}$ and $\mathrm{Q}$ is performed such that error covariance $\mathrm{P}$ and Kalman gain K converges quickly to give optimum solution. 


\subsubsection{Kalman Filter Smoothing}

Smoothing technique is used to improve predictions made by Kalman filter and it uses all the observations till N. Figure 4.5 shows three subdivisions of optimal estimation [50]. The hatched block represents measurement data which includes future as well as past measurement estimates predicted by Kalman filter algorithm. We have the system model as described in equation (4.1), (4.3) with $w_{k}$ and $v_{k}$ as process noise and measurement noise of covariance vectors $\mathrm{Q}$ and $\mathrm{R}$.

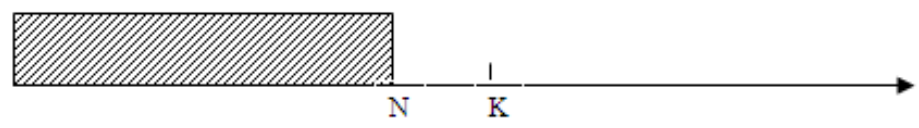

(a) Prediction $\mathrm{K}>\mathrm{N}$

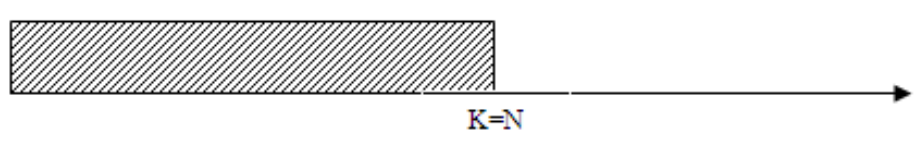

(b) Filtering $\mathrm{K}=\mathrm{N}$

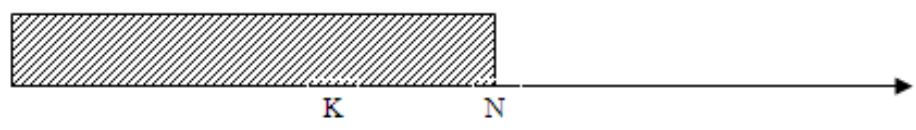

(c) Smoothing $\mathrm{K}<\mathrm{N}$

Figure 4.4 Optimal Estimation Characteristics [50]

The process noise covariance present and past data is stored to use for calculating smoothed data points.

Thus,

$P_{f, k}^{-}=F_{k-1} P_{f, k-1}^{+} F_{k-1}^{T}+Q_{k-1}$

Where $P_{f, k}^{-}$is the covariance vector's updated value and $P_{f, k}^{+}$is the predicted value. 
The forward Kalman gain can be calculated as,

$$
K_{f, k}=P_{f, k}^{+} H_{k}^{T} R_{k}^{T}
$$

The update and predicted state vector values can be calculated as,

$\hat{x}_{f, k}^{-}=F_{k-1} \hat{x}_{f, k-1}^{+}+G_{k-1} u_{k-1}$

$\hat{x}_{f, k}^{+}=\hat{x}_{f, k}^{-}+K_{f, k}\left(y_{k}-H_{k} \hat{x}_{f, k}^{-}\right)$

Thus,

$$
P_{f, k}^{+}=\left(I-K_{f, k} H_{k}\right) P_{f, k}^{-}
$$

There are three prominent smoothers based on observed time constraints,

A) Fixed Lag smoothers: It is used for real time application with latency in the estimation. The performance depends upon the lag time.

B) Fixed Interval Smoothers: It uses entire time period with the cost of offline processing. It runs in forward backward two filter configuration. It estimates each state $x_{k}$ with all measurements for time index, $k=0,1,2, \cdots N$.

C) Fixed point Smoothers used if the estimate needed for particular time instant rather than the whole interval.

The well-known fixed interval smoother, called as RTS smoother presented by Rauch et.al [51] is used in this work. RTS smoother uses the co variances calculated by Kalman filter in its forward pass as an input to the smoothing algorithm and algorithm is run from $\mathrm{N}-1$ to 0 time index in backwards.

For smoothing update, the filter is initialized with the last updated values of $\hat{x}_{f, k}^{+}$and $P_{f, k}^{+}$ $\hat{x}_{s}=\hat{x}_{f, k}^{+}$

Where $\hat{x}_{s}$ is the smoothed state derived from Kalman estimate.

$$
p_{s}=P_{f, k}^{+}
$$


The smoothed covariance estimate is calculated for values of $\mathrm{k}$ ranging from

$$
\begin{aligned}
& k=N-1, N-2, \cdots \cdots 0 ., \\
& I_{f, k+1}^{-}=\left(P_{f, k+1}^{-}\right)^{T}
\end{aligned}
$$

Thus, Kalman gain is calculated by equation,

$K_{s}=P_{f, k}^{+} F_{k}^{T} I_{f, k+1}^{-}$

Finally, the smoothed process noise covariance and smoothed estimate is given by

$$
\begin{aligned}
& P_{s, k}=P_{f, k}^{+}+K_{k}\left(P_{f, k+1}^{-}-P_{s, k+1}\right) K_{k}^{T} \\
& x_{s, k}=x_{f, k}^{+}+K_{k}\left(\hat{x}_{s, k+1}-\hat{x}_{f, k+1}^{-}\right) K_{k}^{T}
\end{aligned}
$$

\subsection{Sensor Fusion Using Kalman Filter}

For reliable position estimation, it is necessary to combine the data from different sensors with the used of software techniques such as fuzzy logic, neural networks, autoregressive models and high power filters. The information received from each sensor must be interpreted properly for optimum estimation. The model used in this research for determining position is known is PVA - Position Velocity Acceleration model.

\subsubsection{Position Velocity Acceleration Model}

Position was obtained by double integrating acceleration data. Newton laws of motion states the relationship between position, velocity and acceleration as, 
$x_{k}=x_{k-1}+v_{k-1} \Delta t+a_{k-1}\left(\Delta t^{2}\right) / 2$

$v_{k}=v_{k-1}+a_{k-1} \Delta t$

$a_{k}=a_{k-1}$

The system states represent the measurement under consideration. Position, velocity and acceleration are concluded as states of the system and can be written in one-dimensional case as,

$$
x_{k}=\left[\begin{array}{l}
p_{k} \\
v_{k} \\
a_{k}
\end{array}\right]
$$

Similar equations can be written for angular acceleration measurement as,

$$
\begin{aligned}
& \theta_{k}=\theta_{k-1}+\omega_{k-1} \Delta t+\alpha_{k-1}\left(\Delta t^{2}\right) / 2 \\
& \omega_{k}=\omega_{k-1}+\alpha_{k-1} \Delta t \\
& \alpha_{k}=\alpha_{k-1}
\end{aligned}
$$

With states,

$$
x_{k}=\left[\begin{array}{c}
\theta_{k} \\
\omega_{k} \\
\alpha_{k}
\end{array}\right]
$$

The measurement and process model for angular acceleration in discrete form can be written as,

$$
\begin{aligned}
& x_{k}=\left[\begin{array}{lll}
0 & 1 & 0 \\
0 & 0 & 1 \\
0 & 0 & 0
\end{array}\right] x_{k-1}+\left[\begin{array}{l}
0 \\
0 \\
1
\end{array}\right] w_{k} \\
& y_{k}=\left[\begin{array}{lll}
1 & 0 & 0
\end{array}\right] x_{k}+v_{k}
\end{aligned}
$$


Equation (4.40) can be represented in matrix form as,

$$
\begin{aligned}
& x_{k}=\left[\begin{array}{ccc}
1 & \Delta t & \Delta t^{2} / 2 \\
0 & 1 & \Delta t \\
0 & 0 & 1
\end{array}\right] x_{k-1}+w_{k} \\
& y_{k}=\left[\begin{array}{lll}
1 & 0 & 0
\end{array}\right] x_{k}+v_{k}
\end{aligned}
$$

Since bias drift error is one of the main contributors in acceleration sensor, process model was built by using acceleration bias as random walk process [21]. Random walk process can be represented by differentiating acceleration signal as,

$\dot{a}_{k}=r w_{k-1}$

Where $r w$ is a white noise with variance $\sigma_{r w}$. Power spectral density, $\mathrm{W}$ is derived by considering variance of equation (4.46) as,

$W=\sigma\left(\dot{a}_{k}\right)$

Variance in terms of power spectral density for each state can be obtained by integrating equation (4.47) as,

$\sigma_{a_{k}}^{2}=\int_{0}^{\Delta t} \int_{0}^{\Delta t} W \times \Delta t \times d(\Delta t) \times d T$

$=\int_{0}^{\Delta t} \frac{W \Delta t^{2}}{2} d T \quad$ Where, $T$ is the total time.

The equation is further reduced to,

$$
\sigma_{a_{k}}^{2}=\frac{W \times \Delta t^{3}}{6}
$$

From equation (4.49) we can conclude that variance increases as a cubic function of time. 
Similarly process noise covariance matrix given by equation (4.26) can be modified for all three states, in terms of integrated white noise density function with variance $\sigma_{a_{k}}^{2}$ and spectral density function as W. as,

$$
Q=\left[\begin{array}{lll}
\frac{W}{20} \Delta t^{5} & \frac{W}{8} \Delta t^{4} & \frac{W}{6} \Delta t^{3} \\
\frac{W}{8} \Delta t^{4} & \frac{W}{3} \Delta t^{3} & \frac{W}{2} \Delta t^{2} \\
\frac{W}{6} \Delta t^{3} & \frac{W}{2} \Delta t^{2} & W \Delta t
\end{array}\right]
$$

For simplicity, all the equations are stated in one-dimensional format. A similar model is built for two-dimensional measurement with all 14 states explained in appendix A.1.

\subsection{Bias Drift Error Model Using Kalman Filter}

Accelerometer bias is defined as output of acceleration sensor when no acceleration input is present. Bias can be negative or positive depending upon the manufacturing characteristics. It is presented in $g$ units. Bias drift is one of the critical parameter to control in case of acceleration sensor. This error is again accumulated when we integrate acceleration reading for distance measurement.

This can be explained by equation,

$$
\text { Position }_{\text {error }}=\frac{1}{2} * \text { Bias }_{\text {Drift }} * \text { time }^{2}
$$

At $100^{\text {th }} \mathrm{sec}$, with bias error of $0.1 \mathrm{~m} / \mathrm{s}^{2}$, the expected position error would be $500 \mathrm{~m}$. The condition gets worse as the bias error and time increases. Thus, compensation for bias drift is essential. A stationary, Gauss-Markov process was used to examine the 
acceleration bias drift. A deterministic, nonlinear parametric model was used to analyse the accelerometer data based on Levenberg Marquardt iterative least square fitted method [52] as,

$$
\varepsilon_{\text {model }}(t)=a_{1}\left(1-e^{-t / T}\right)+a_{2}
$$

$\varepsilon_{\text {mode } l}(t)$ is the fitted bias drift error model for acceleration sensor. $a_{1}, a_{2}$ and $\mathrm{T}$ are the parameters to be tuned. Single $Y$ axis acceleration data was recorded over period of two hours. The above model was validated with Levenberg Marquardt fit test by using LabVIEW programming on acquired data. Figure 4.5 shows acceleration bias data and Levenberg Marquardt fitted curve. The model parameters were selected according to best fit. The validity of model was checked by applying autocorrelation function on residuals. Figure 4.6 shows that $97 \%$ of the sample data falls within the error bounds. From the autocorrelation function, it was observed that the residuals of fitted error model passed whiteness test with average variance value approximate to unity. The power spectral density $\mathrm{W}$, was set to one. Table 4.1 shows tuned parameter values of equation 4.52. 


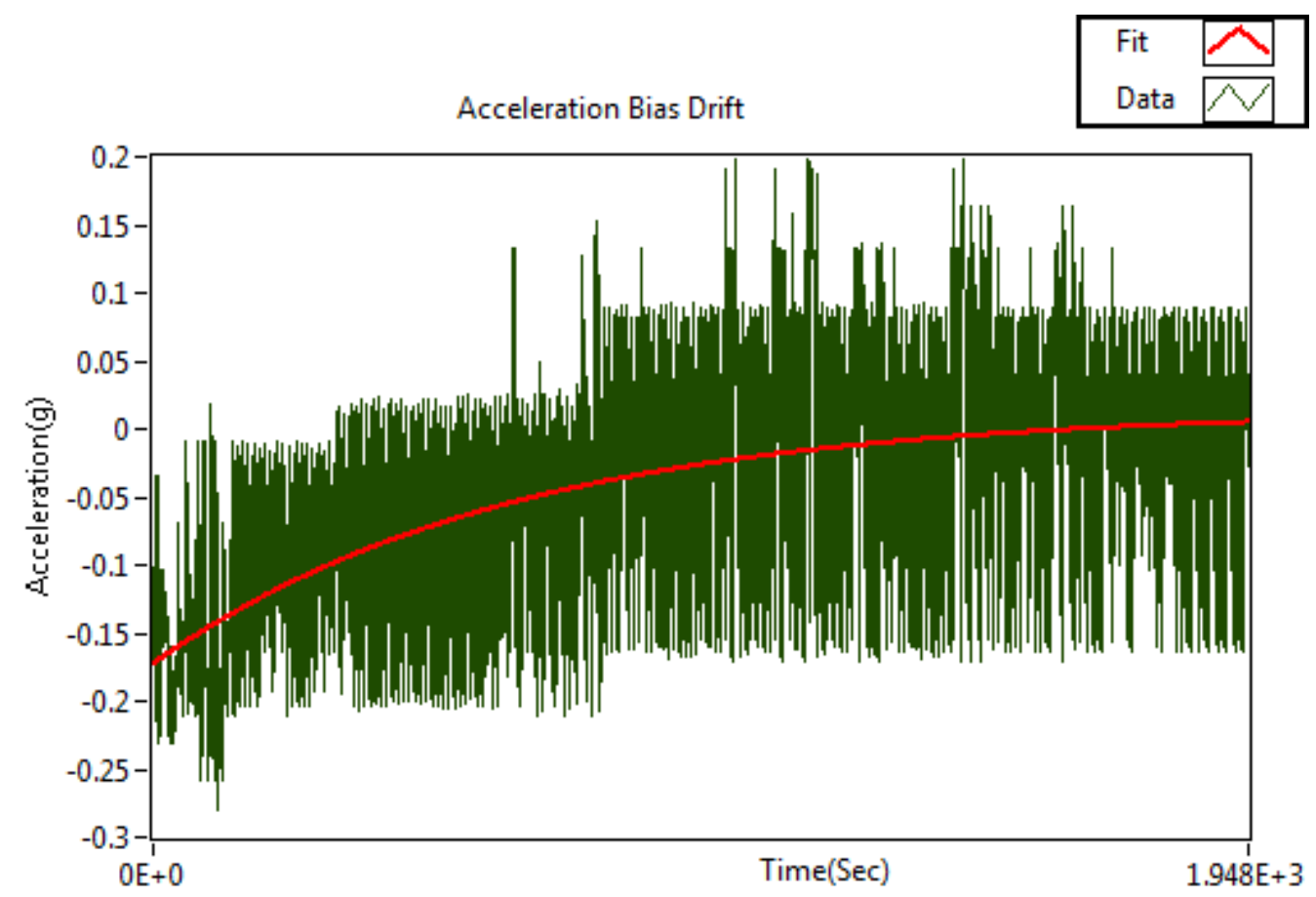

Figure 4.5 Acceleration Bias Data with Levenberg Marquardt Fitted Curve

Table 4.1 Levenberg Marquardt Fitted Parameters

\begin{tabular}{|l|l|}
\hline Parameter & Fitted Value \\
\hline$a_{1}$ & 0.25 \\
\hline$a_{2}$ & -0.21 \\
\hline$T$ & 70 \\
\hline
\end{tabular}




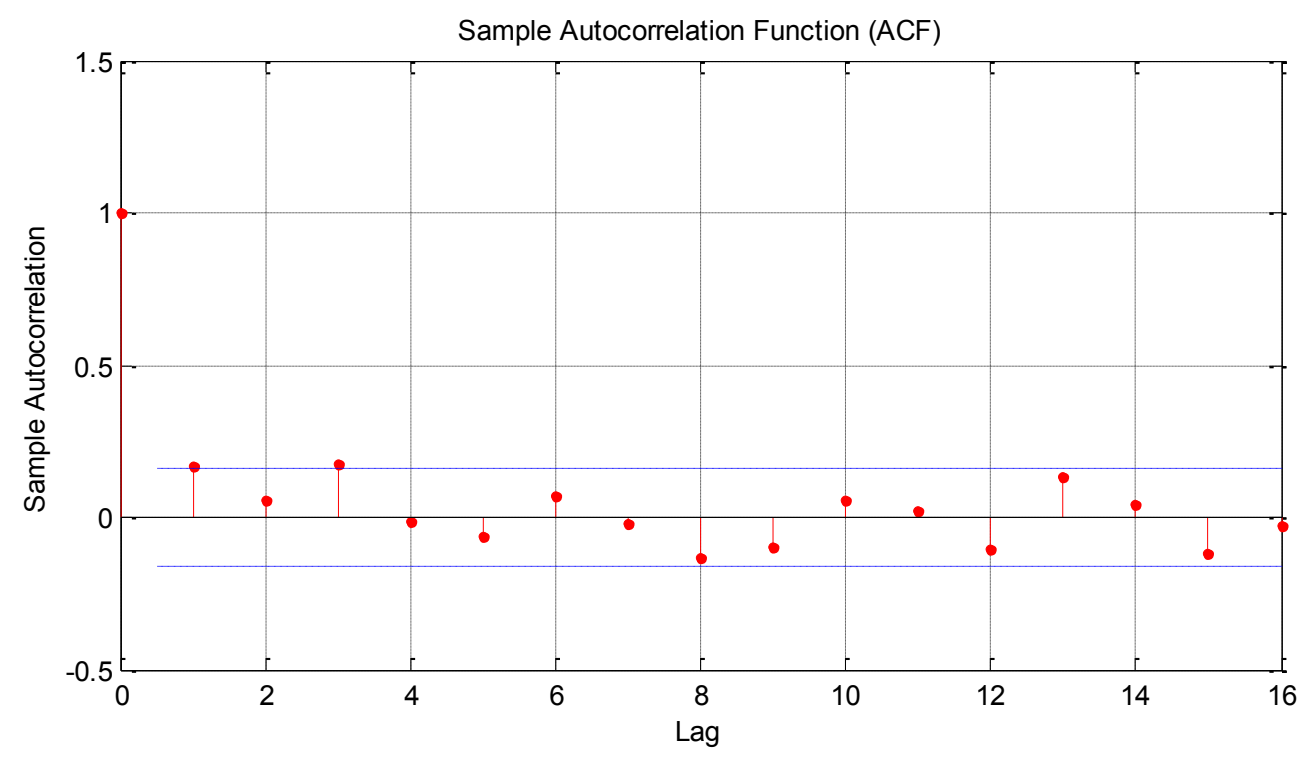

Figure 4.6 Autocorrelation of Residuals

System states are modified by including bias error state in the matrix given by equation (4.53) as,

$$
x_{k}=\left[\begin{array}{l}
x_{(}(k) \\
v_{x}(k) \\
a_{x}(k) \\
\varepsilon_{a}(k)
\end{array}\right]
$$

Deterministic input, also known as control input is set as fitted error equation,

$$
u_{a}=\left[\begin{array}{l}
0 \\
0 \\
0 \\
\frac{\Delta t\left(a_{1}+a_{2}\right)}{T+\Delta t}
\end{array}\right]
$$

State transition matrix can be stated as, 


$$
A=\left[\begin{array}{cccc}
1 & \Delta t & 0.5 \Delta t^{2} & 0 \\
0 & 1 & \Delta t & 0 \\
0 & 0 & 1 & 0 \\
0 & 0 & 0 & \frac{\operatorname{Tax}}{\operatorname{Tax}+\Delta t}
\end{array}\right]
$$

And finally, observation matrix contain acceleration measurement as,

$$
C=\left[\begin{array}{llll}
0 & 0 & 1 & 0
\end{array}\right]
$$

Each row relates to the states of the system. For measuring acceleration, matrix value was set to 1 . Thus the update will be done for acceleration and state transition matrix predicts the position and velocity accordingly.

\subsection{Angular Acceleration Measurement}

Angular acceleration is measured by linear acceleration sensors by following equations [36]

$$
\begin{aligned}
& a_{1}=G[-g \sin (\theta)]+K[R \alpha-g \cos (\theta)]+\varepsilon_{\mathrm{mod} l}(t) \\
& a_{2}=G[g \sin (\theta)]+K[R \alpha+g \cos (\theta)]+\varepsilon_{\mathrm{mod} l}(t)
\end{aligned}
$$

$\mathrm{G}$ is the sensitivity along the main axis, $\theta$ represents angle between radius $\mathrm{R}$ and earth gravity component, ' $\mathrm{g}$ '. The gravity component in equation 4.58, $g \cos (\theta)$ results from earth's gravitational force and is depend upon orientation of acceleration sensor with respect to earth gravitational force. If the acceleration sensing axis placed in opposite direction the gravity factor can be minimised since it's not possible to completely eliminate the effect of gravity on the sensor. The sensitivity term will affect both the 
accelerometer sensors equally in opposite direction thus the term was ignored. The simplified equations can be written as:

$a_{1}=R \alpha+g \cos (\theta)$

$a_{2}=R \alpha-g \cos (\theta)$

$\alpha=\frac{a_{1}+a_{2}}{2 R}$

The sensor ADXL335 measures linear acceleration. From the basic laws of physics we know:

Angular acceleration $=$ Radius * Linear Acceleration.

Thus, angular acceleration is directly proportional to the distance from the axial. Since linear accelerometers are of small size and easily available they can be used for sensing angular acceleration [14] the rotational kinematics doesn't allow to reset the measurement after every 360 degrees thus, the angles where recorded without subtracting 360 after each rotation.

The state space representation for angular acceleration measurement is as shown;

$$
\left[\begin{array}{l}
\theta \\
\omega \\
\alpha \\
b
\end{array}\right]=\left[\begin{array}{ccccc}
1 & \Delta t & \Delta t^{2} / 2 & 0 \\
0 & 1 & \Delta t & 0 \\
0 & 0 & 1 & 0 \\
0 & 0 & 0 & T /(T+\Delta t)
\end{array}\right]\left[\begin{array}{l}
\theta \\
\omega \\
\alpha \\
b
\end{array}\right]+\left[\begin{array}{l}
0 \\
0 \\
1 \\
\frac{\Delta t(c 1+c 2)}{(T+\Delta t)}
\end{array}\right] \alpha_{\text {measured }}
$$

With observation matrix as,

$c=\left[\begin{array}{llll}0 & 0 & 1 & 1\end{array}\right]$

Bias drift error variance is added in uncertainty as explained by process covariance matrix, Q. Thus matrix $\mathrm{Q}$ can be expressed as, 


$$
Q=\left[\begin{array}{cccc}
\frac{W}{20} \Delta t^{5} & \frac{W}{8} \Delta t^{4} & \frac{W}{6} \Delta t^{3} & 0 \\
\frac{W}{8} \Delta t^{4} & \frac{W}{3} \Delta t^{3} & \frac{W}{2} \Delta t^{2} & 0 \\
\frac{W}{6} \Delta t^{3} & \frac{W}{2} \Delta t^{2} & W \Delta t & 0 \\
0 & 0 & 0 & W \sigma_{\varepsilon}^{2}
\end{array}\right]
$$

The RMS acceleration noise can be calculated by multiplying power spectrum density by bandwidth. It provides noise as a function of frequency. Spectral power density for white noise is nearly constant within a fixed bandwidth. The value was assumed as one rad per second. The Kalman filter algorithm was implemented with bias error drift model and RTS smoothing technique using above equations. 


\section{CHAPTER 5}

\section{Results and Discussion}

This chapter begins with the evaluation of the accelerometer bias observed for error compensation modelling. Furthermore, the position estimation was calculated based on bias drift readings. The chapter further discusses results of rotational measurement and the effect of different rotational speeds on error characteristics.

\subsection{Accelerometer Bias Test for Error Compensation}

To evaluate the performance of acceleration sensor, it is necessary to check the drift error pattern in stationary state. The small random output changes observed in the reading is due to instability inside the acceleration sensor. The random error is also characterized by noise power spectrum density.

\subsubsection{Static Bias Test}

To observe the bias drift when sensor is not rotating, static bias test was performed. As shown in the figure 5.1, the sensor was kept steady at $0^{\circ}$ orientation for two hours to observe the bias drift error. $\mathrm{X}$ axis data was acquired at the sampling rate of $1 \mathrm{KHz}$. Bias error is dependent on the temperature and it also has variable output with power supply.. Figure 5.2 shows acceleration drift for sensor A1-X axis measurement. 


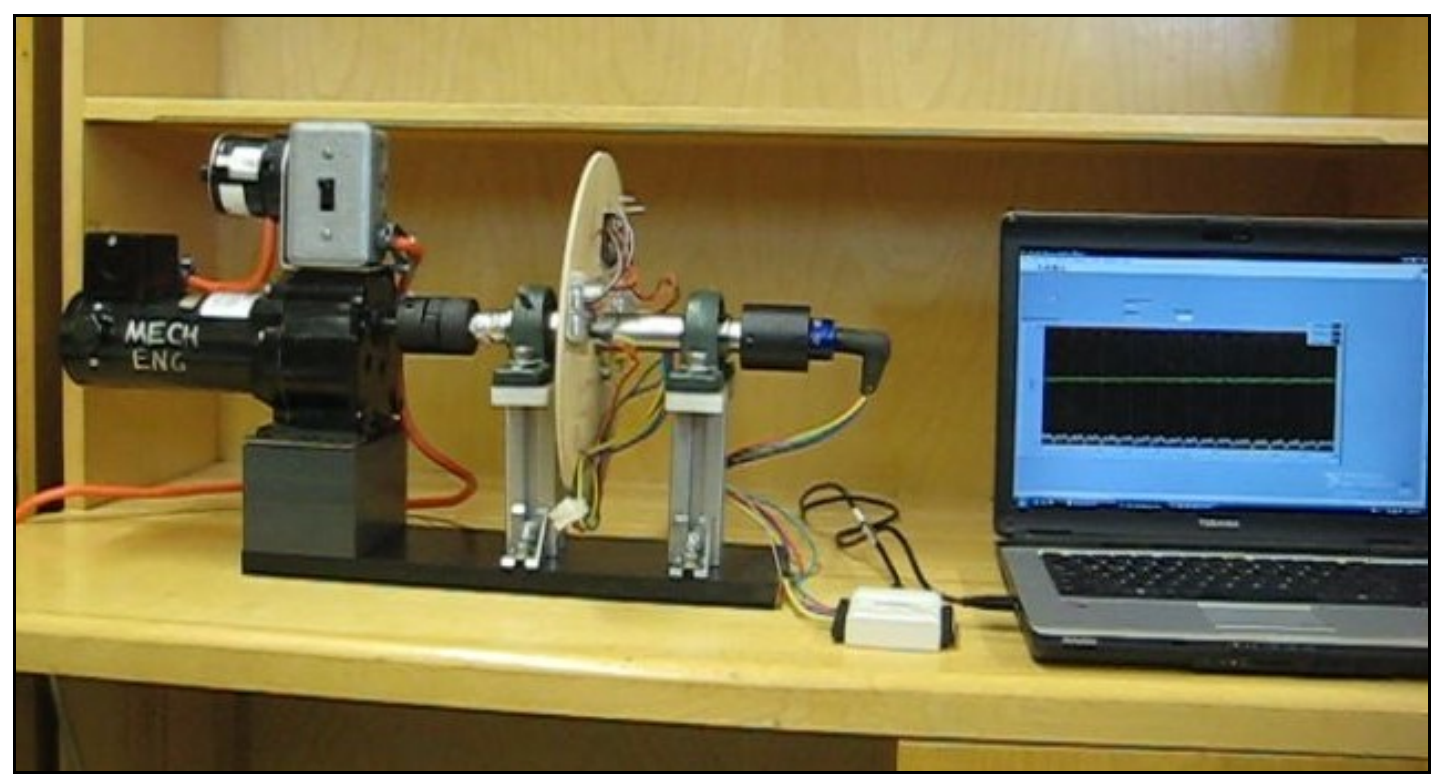

Figure 5.1 Mechanical Design

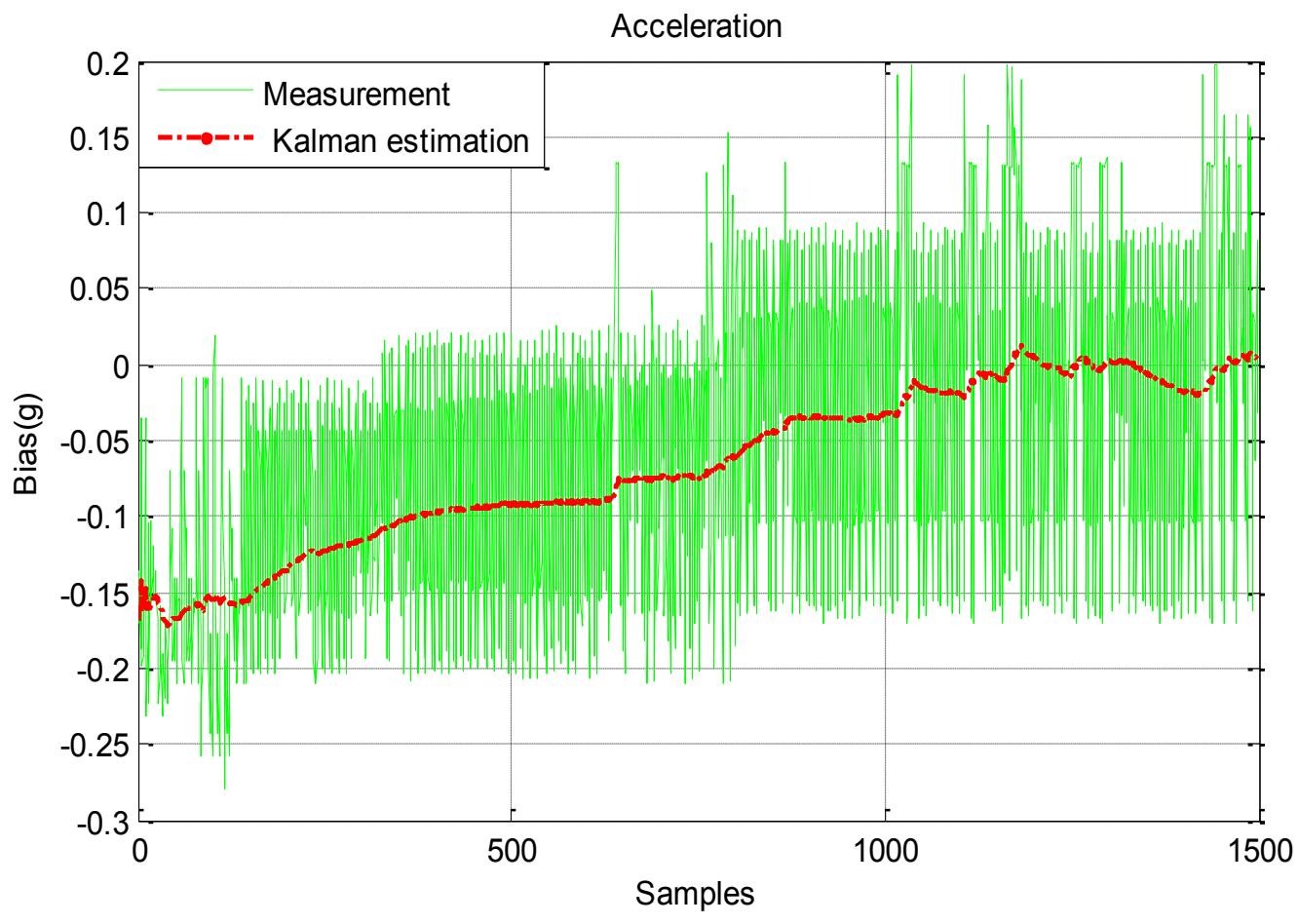

Figure 5.2 Static Bias Test- (Data Down Sampled by 25) 
When the sensor is static, data is recorded for two hours and down sampled by 25 for plotting. Similar test was performed for both the axes of acceleration sensor A1 and A2. Figure 5.3 shows the bias error drift for all the axes estimated by Kalman filter error model.

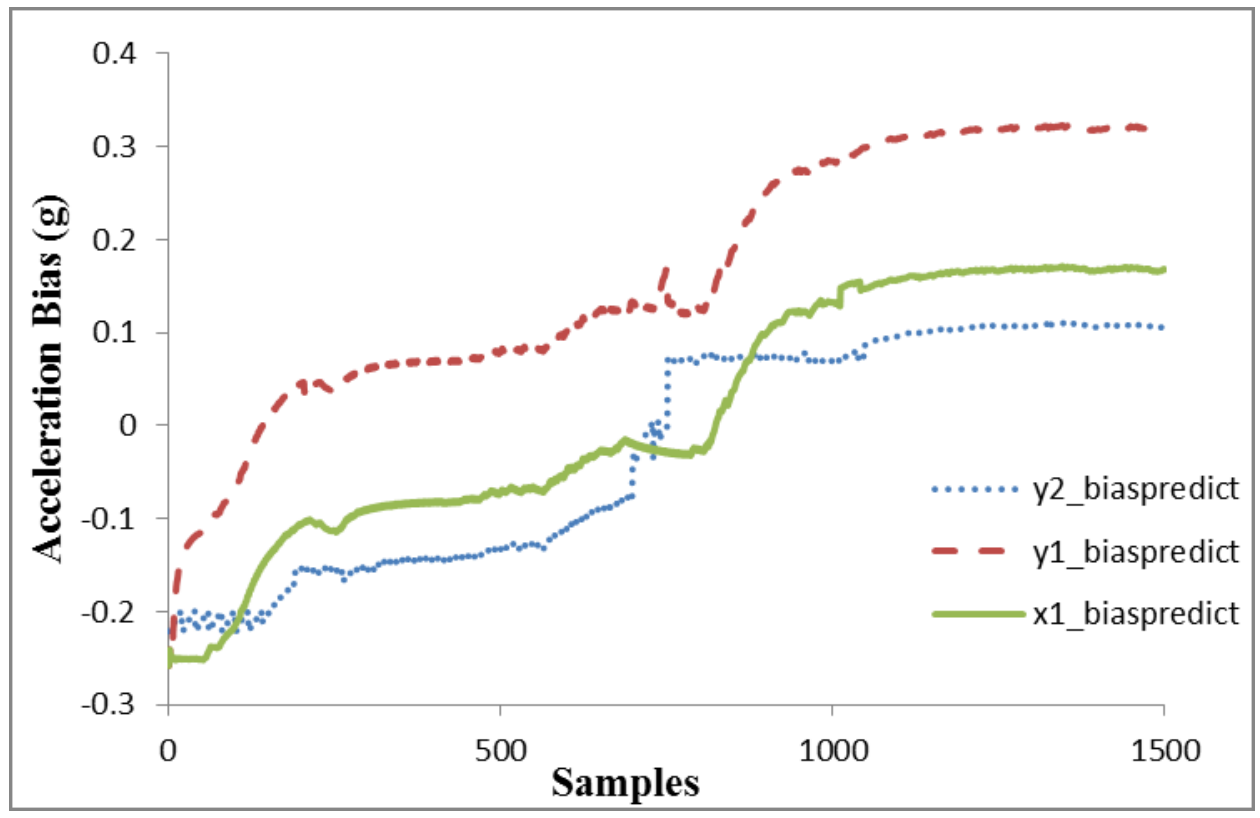

Figure 5.3 Bias Drift Estimated by Kalman Filtering

As shown in the figure 5.2, the bias stabilises after few seconds and filter provides fairly good noise reduction. The output was recorded for 2 hours with steady state, and only gravity acting on the sensor. The recorded data for $x_{2}$ axis is as shown in the figure 5.2. The standard deviation obtained is the accelerometer bias drift. Fitted data equation (4.42) is used to set the bias drift error model as explained in Chapter 4.

\subsubsection{Angular Bias Test}

Angular bias test is performed with acceleration sensor mounted on a rotating platform as shown in the figure 5.1. Earth's gravity is taken as a measurement for calibrating accelerometer. The test is also known as 4 point tumble test [53]. ADXL335 has a ratio 
metric characteristic. It means that the output changes depending upon the supply voltage. The supply voltage provided for this project is 3.3 volts thus, for $0 \mathrm{~g}$ acceleration, expected voltage output is $3.3 / 2=1.65$ volts. Bias is calculated by taking the difference between ideal curve and measured data curve at $0^{\circ}$ and $180^{\circ}$ [19] [28].

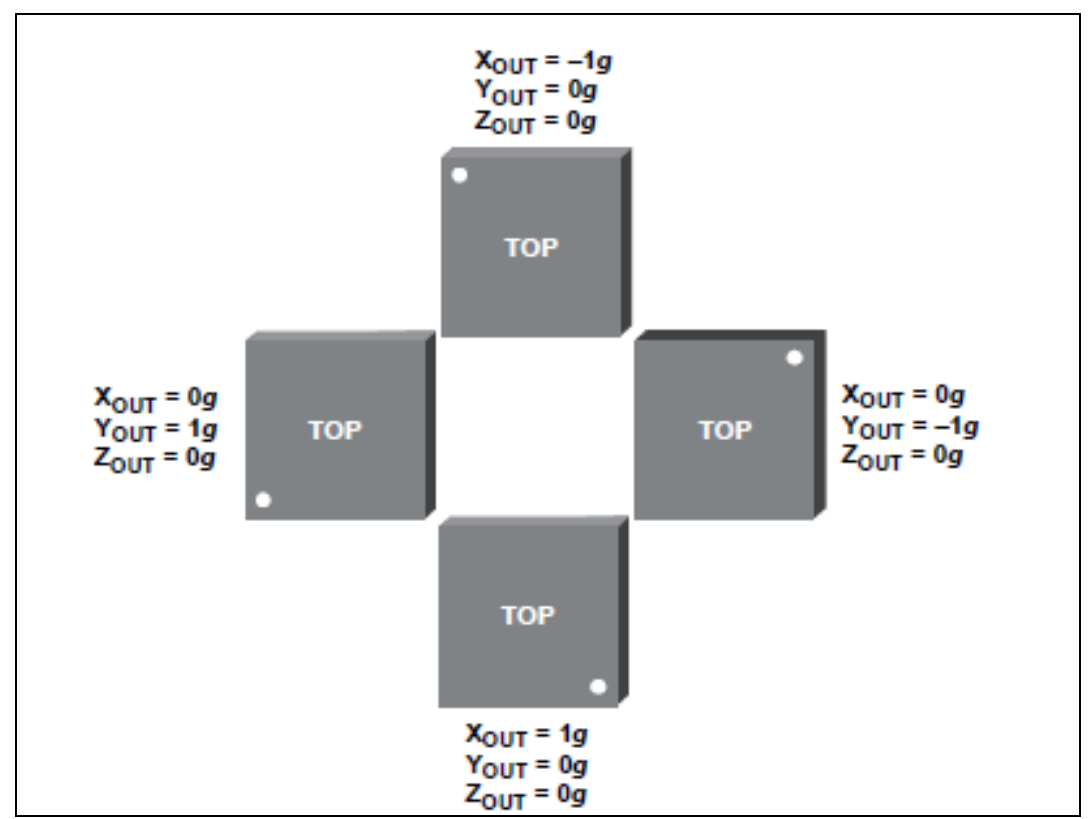

Figure 5.4 Output Response Vs. Orientation [35]

Thus, for every $1 \mathrm{~g}$ change the change in the voltage is $0.33 \mathrm{~V}$. As shown in the adxl data sheet, for power supply of 3.3 volts, the change in output for $1 \mathrm{~g}$ is nearly 0.33 volts. At $0^{\circ}, \mathrm{x}$ axis $=0 \mathrm{~g}$ and $\mathrm{y}$ axis $=-1 \mathrm{~g}$. Thus, voltages levels would be $\mathrm{x} \_$voltage $=1.65$ volts or $0 \mathrm{~g}, \mathrm{y}_{-}$voltage $=1.65+0.33=1.98$ volts. Four angles were selected such as $0,90,180,270$ and 360 to test the bias effect when accelerometer sensor is rotating on the wheel. Readings were taken for $\mathrm{x}$ and y axes of the adxl 335 accelerometer sensor. Figure 5.5 shows raw data of both the axes for complete 360 degree rotation with readings taken at the intervals of $90^{\circ}$. 


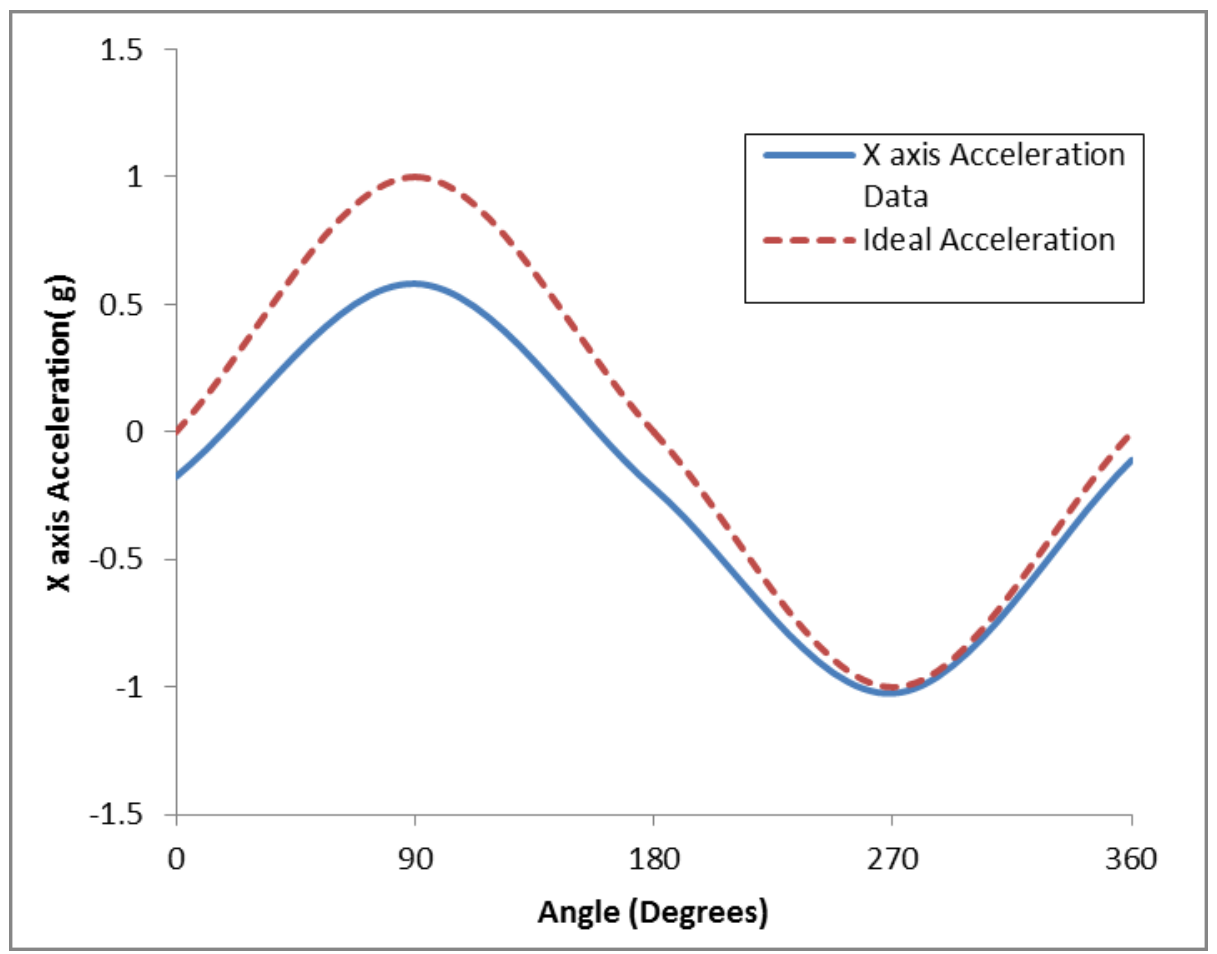

Figure 5.5 Ideal and Measured Waveform of X axis with Power Supply of 3.3 Volts.

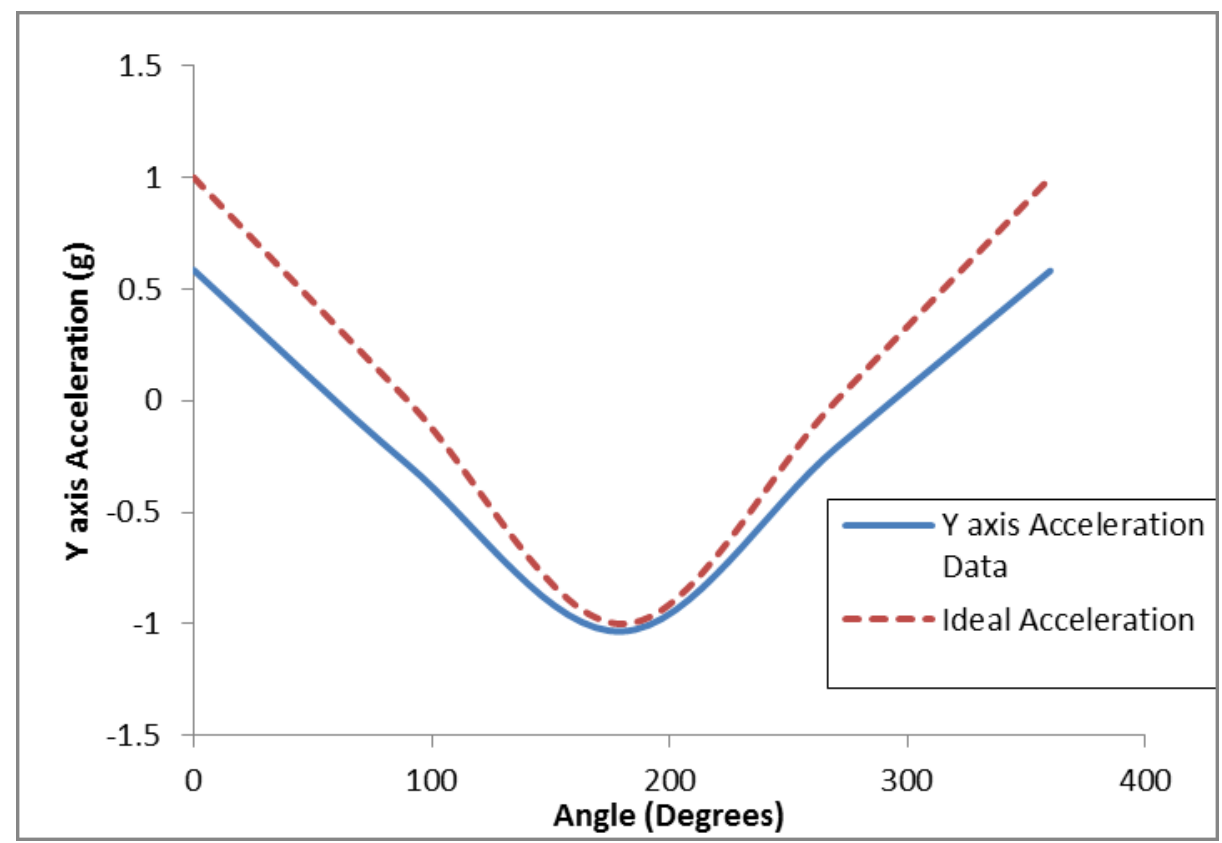

Figure 5.6 Ideal and Measured Waveform of Y axis with Power Supply of 3.3 Volts 
To observe the effect of bias drift, data was collected for several rotations on different days.

The table 5.1 summarizes the bias drift observed in both axes of ADXL 335 sensor.

$$
\text { bias }_{\text {drift }}=\frac{\text { Acceleration }_{0^{0}}+\text { Acceleration }_{180^{\circ}}}{2}
$$

Thus,

bias $_{\text {drift }}=\frac{-0.17517+(-0.22057)}{2}$

bias $_{\text {drift }}=-19.78$

Thus, correction factor for offset $=-19.78 \mathrm{mg}$

Similar experiment was performed for A2 sensor. The bias drift observed was $19.56 \mathrm{mg}$.

For y axis,

bias $_{\text {drift }}=\frac{0.58541+(-1.0330)}{2}$

bias $_{\text {drift }}=22.38$

Offset correction of $22.38 \mathrm{mg}$ was observed with A2 sensor.

Similarly, Scale factor can be calculated as,

$$
m 1=\frac{\text { Acceleration }_{90^{0}}-\text { Acceleration }_{270^{\circ}}}{2}
$$

And correction factor for scale error Ks:

$$
K_{s}=\frac{m}{m 1} \text { Where, } \mathrm{m}=\text { Ideal Sensitivity. }
$$

Bias drift error and scale factor error values were used for calibration. 
Table 5.1 ADXL335- Bias and Scale Factor Error

\begin{tabular}{|l|l|l|l|}
\hline \multicolumn{2}{|l|}{ X axis (mean) } & \multicolumn{2}{l|}{ Y axis(mean) } \\
\hline Bias(mg) & S.F.(\%) & Bias(mg) & S.F.(\%) \\
\hline 19.68 & 0.0041 & 22.29 & 0.0869 \\
\hline
\end{tabular}

\subsection{Simulation Model}

The rotation test generates sinusoidal function for $\mathrm{x}$ axis and cosine function for $\mathrm{y}$ axis as shown in figure 5.5 and figure 5.6. The Position-Velocity-Acceleration three dimensional model was simulated in MATLAB. Measurement was added with random noise for simulations and compared with true data. The acceleration was simulated by using sin and cosine function as, $a_{x}=\sin \left(t_{k}\right)$ and $a_{y}=\cos \left(t_{k}\right)$.Measurement noise and acceleration process noise deviation settings were changed to observe the effect of $\mathrm{Q}$ and $\mathrm{R}$. Total simulation time duration was set to 100 seconds with time step of 0.2 second.

\subsection{Effect of $Q$ and $R$}

By changing the process noise covariance and measurement noise covariance matrix, effects of $\mathrm{Q}$ and $\mathrm{R}$ has been observed on the Kalman estimation. The setting was used to tune the filter for real time measurements. Initially value of error covariance matrix P is set high such as 100. R and Q values where selected as four and one respectively. The graph shows simulated acceleration measurement and Kalman estimation by red line. Corresponding Kalman gain and error covariance convergence graphs are as shown in Figure 5.8 and Figure 5.10 respectively. It is observed that, when $\mathrm{Q}>\mathrm{R}$, Filter detects frequent changes in measurement more accurately than setting $Q$ smaller than Measurement Uncertainty R. If the measurement uncertainty is large, Kalman estimate 
doesn't rely on measured data. Instead it uses error covariance to correct the measurement. To evaluate this assumption, $\mathrm{R}$ and $\mathrm{Q}$ values were set to four and one respectively. The simulation confirmed that filter did not follow the simulated acceleration when $\mathrm{R}>\mathrm{Q}$ as shown in figure 5.9.

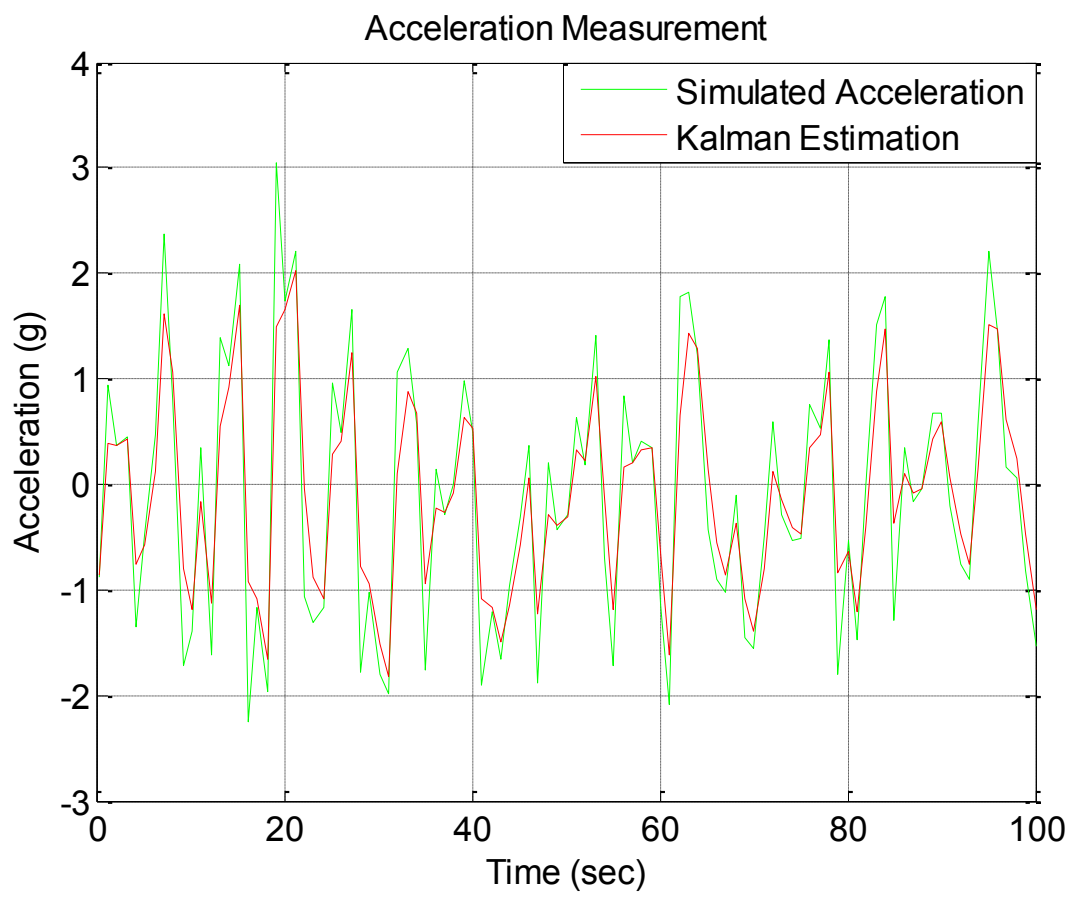

Figure 5.7 Simulated Acceleration data, $\mathrm{Q}>\mathrm{R}$ 


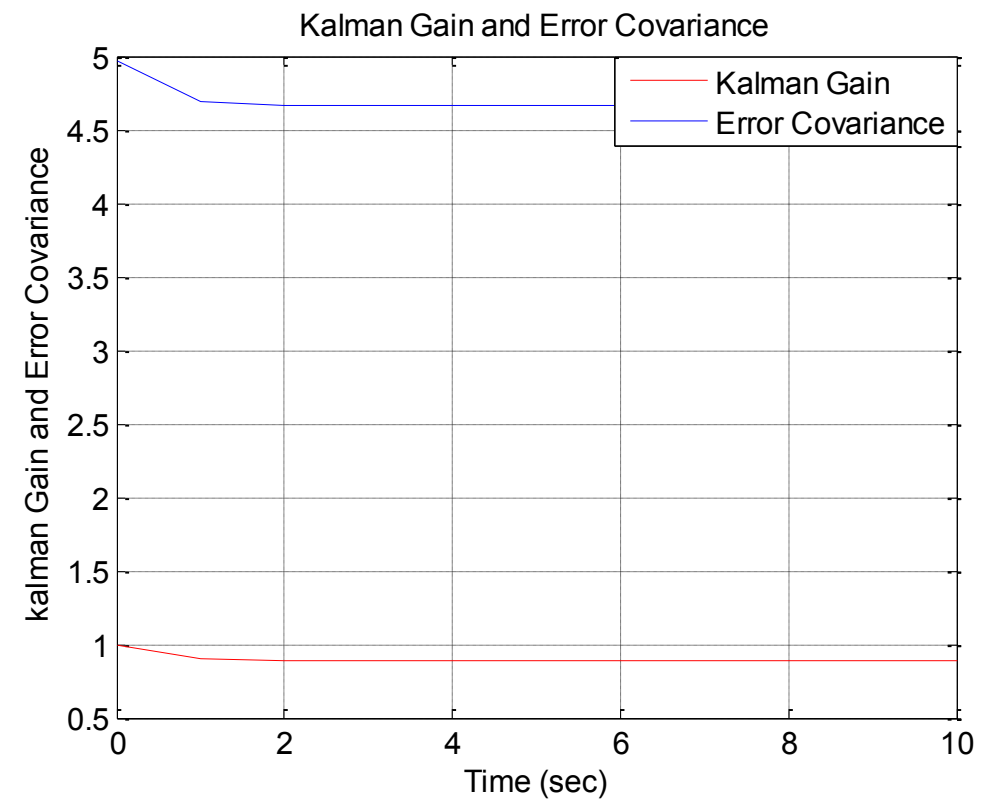

Figure 5.8 Kalman gain and Error Covariance when Q > R

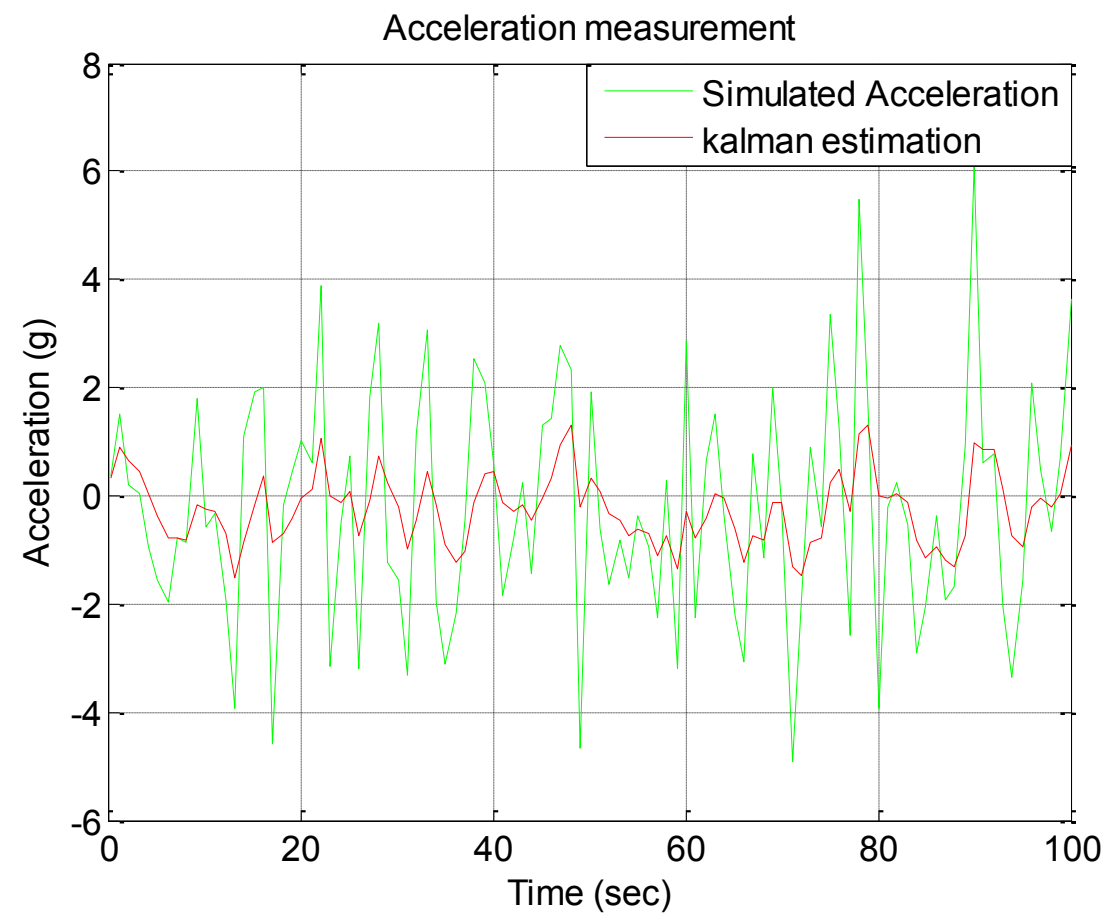

Figure 5.9 Simulated Acceleration Data $Q<R$ 


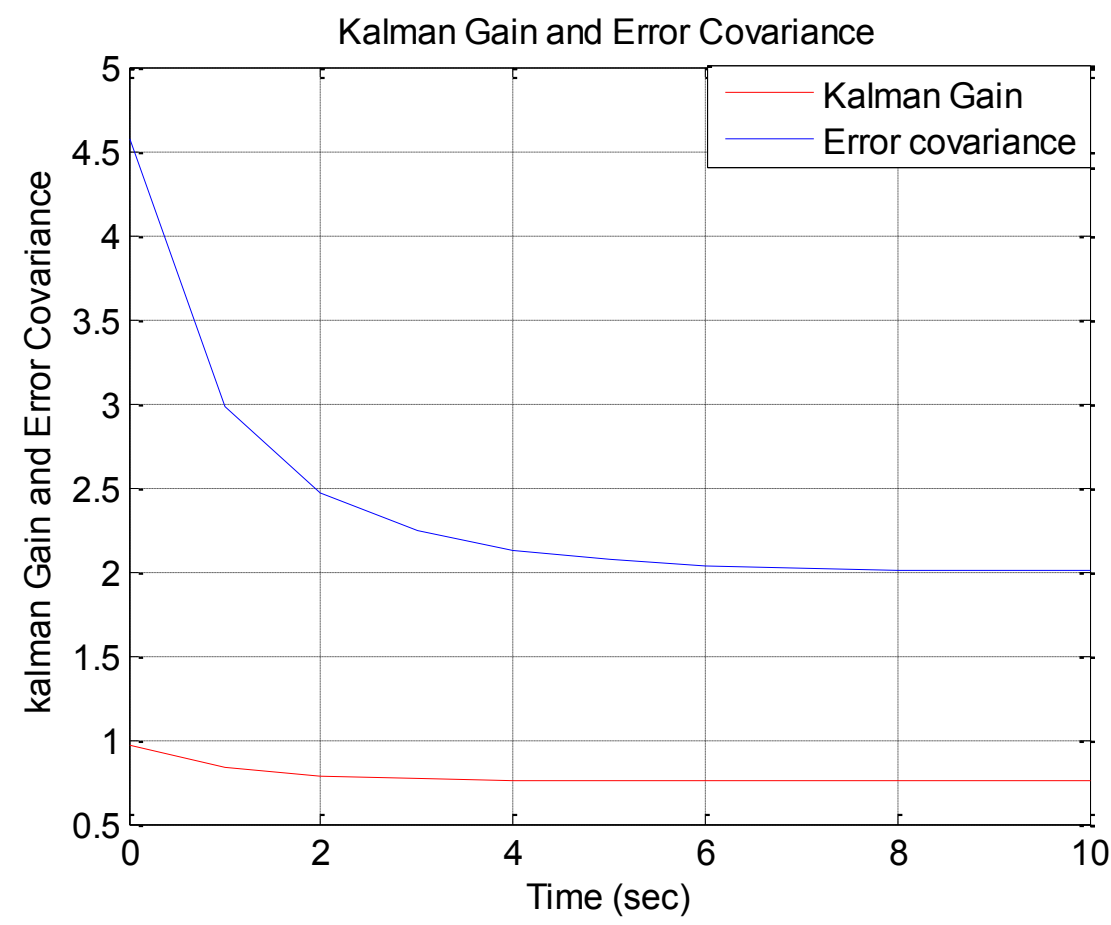

Figure 5.10 Error Noise Covariance and Kalman Gain $Q<R$

Figure 5.10 suggests disordered response of filter to rapid changes in the measurement for first ten seconds. It takes approximately eight seconds to settle the error in the measurement which reflects measurement uncertainty. On the other hand, with large Q value, the error settles within two seconds and filter converges quickly.

\subsection{Linear Acceleration Measurement}

The data was collected from USB 6009 data acquisition card and stored in the file for further processing. The mechanical test ring used is as shown in the figure 5.11 


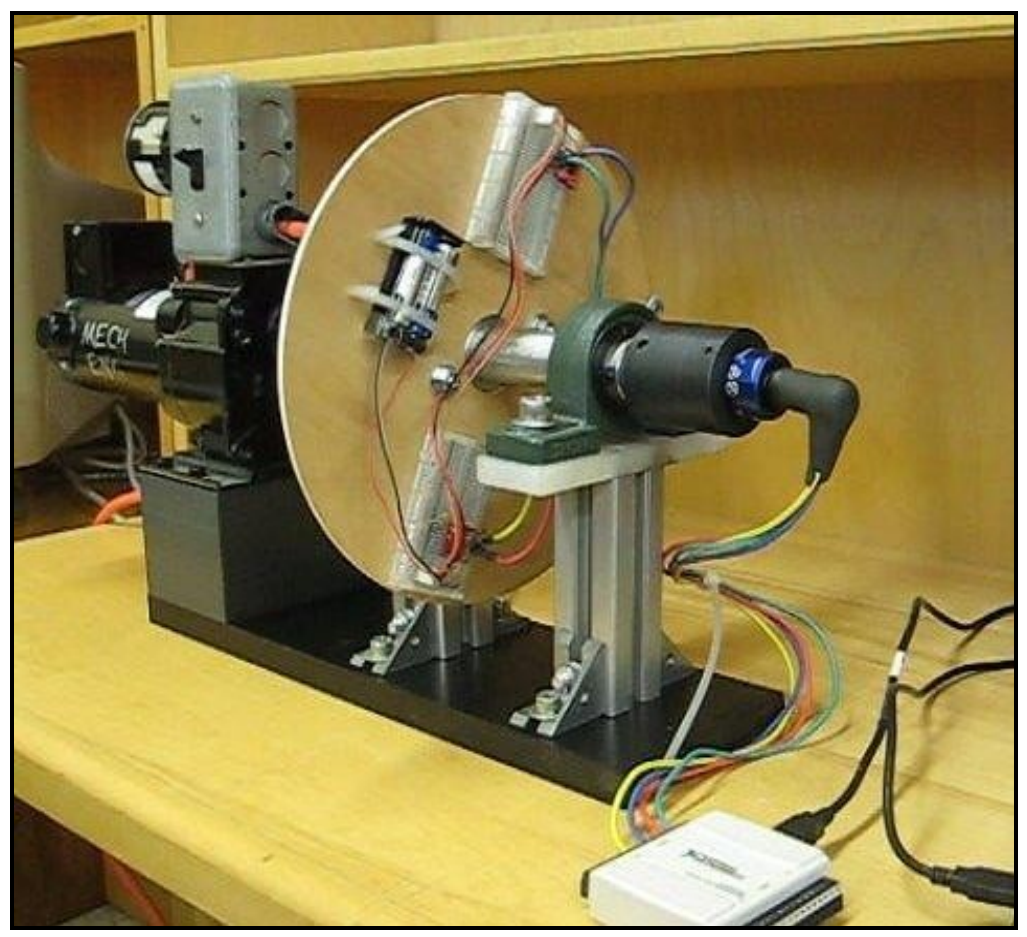

Figure 5.11 Rotating Wheel with Electrical Connector

\subsubsection{Roll Measurement}

With sampling rate of $200 \mathrm{~Hz}$, y axis raw acceleration data for $A_{1}$ and $A_{2}$ was recorded and fused as shown in the figure 5.12. 


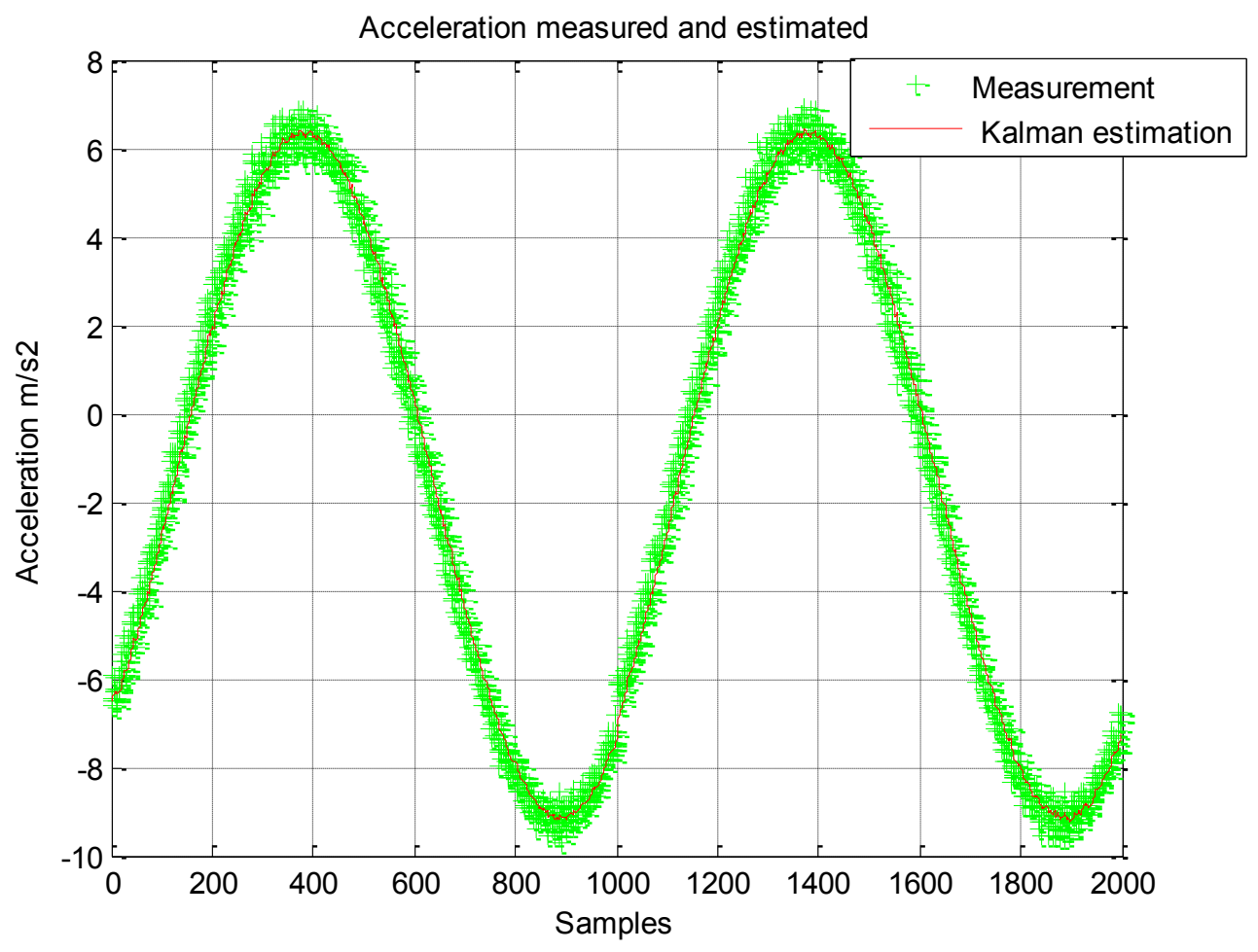

Figure $5.12 y_{1}$ and $y_{2}$ fused raw data

It was observed that the raw data contain evident white noise at low frequency of $200 \mathrm{~Hz}$. Kalman filter error model reduces this noise as shown by red solid line. By fusing raw acceleration data, position was predicted as shown in the figure 5.13.

When the wheel completes one revolution the calculated true position would be $(\mathrm{R}=10$ thus $2 * \mathrm{pi} * \mathrm{R}=62.83 \mathrm{~cm}=0.62 \mathrm{~m}$ for first five seconds. The data was recorded with frequency of $200 \mathrm{~Hz}$. Figure 5.13 shows position estimation comparison between double integration method, Kalman estimation method and Kalman-Smoother estimation. With Kalman filter the error observed at 2 seconds, was 3.58 meters as compared to error of 6.88 meters by double integration. 


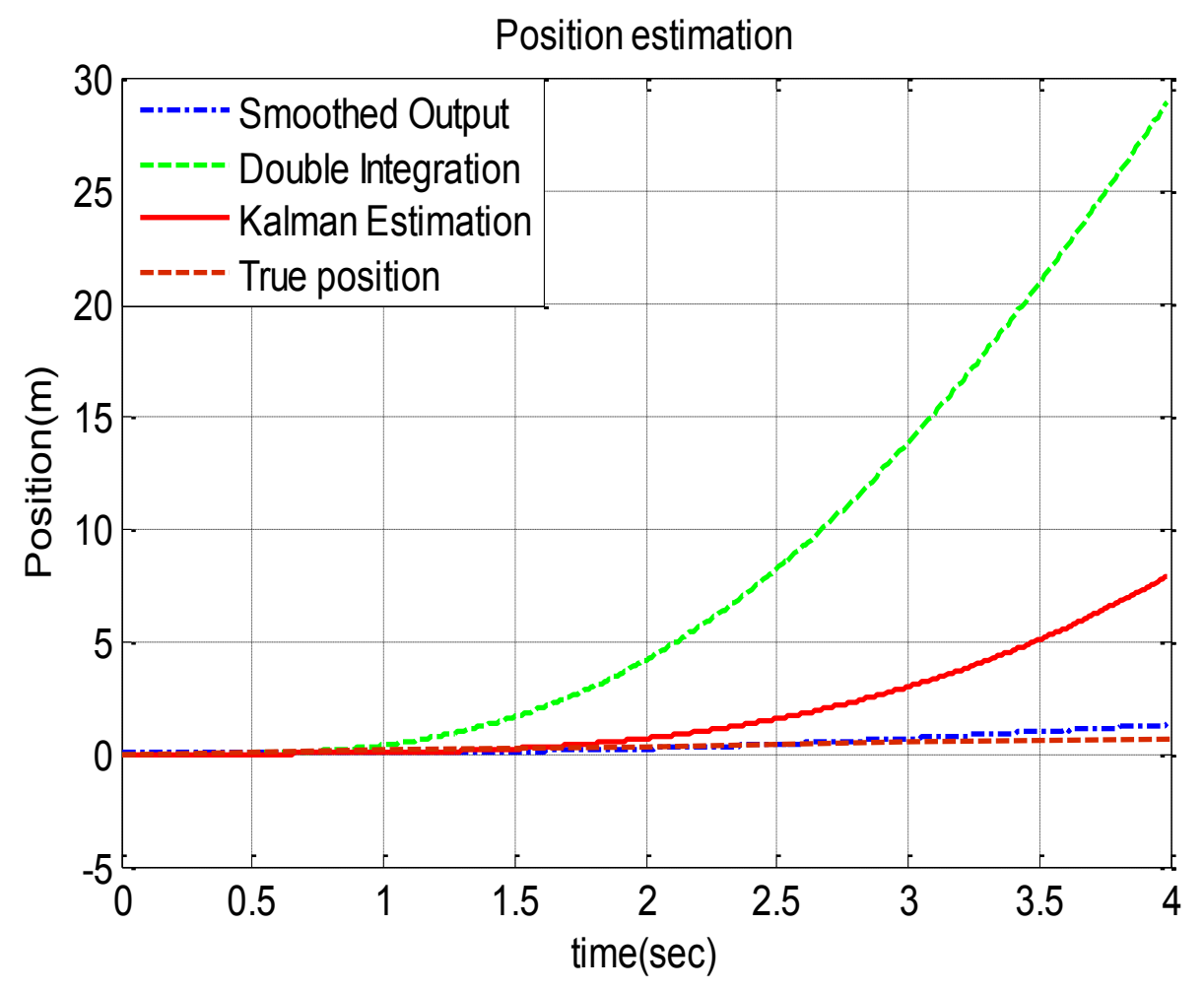

Figure 5.13 Position Estimation by fusing $y_{1}$ and $y_{2}$ data

The best position estimation was obtained by introducing smoother algorithm in the Kalman filter estimation. As shown in figure 5.13 with smoothing technique, error was reduced to six meter. 


\subsubsection{Pitch Measurement}

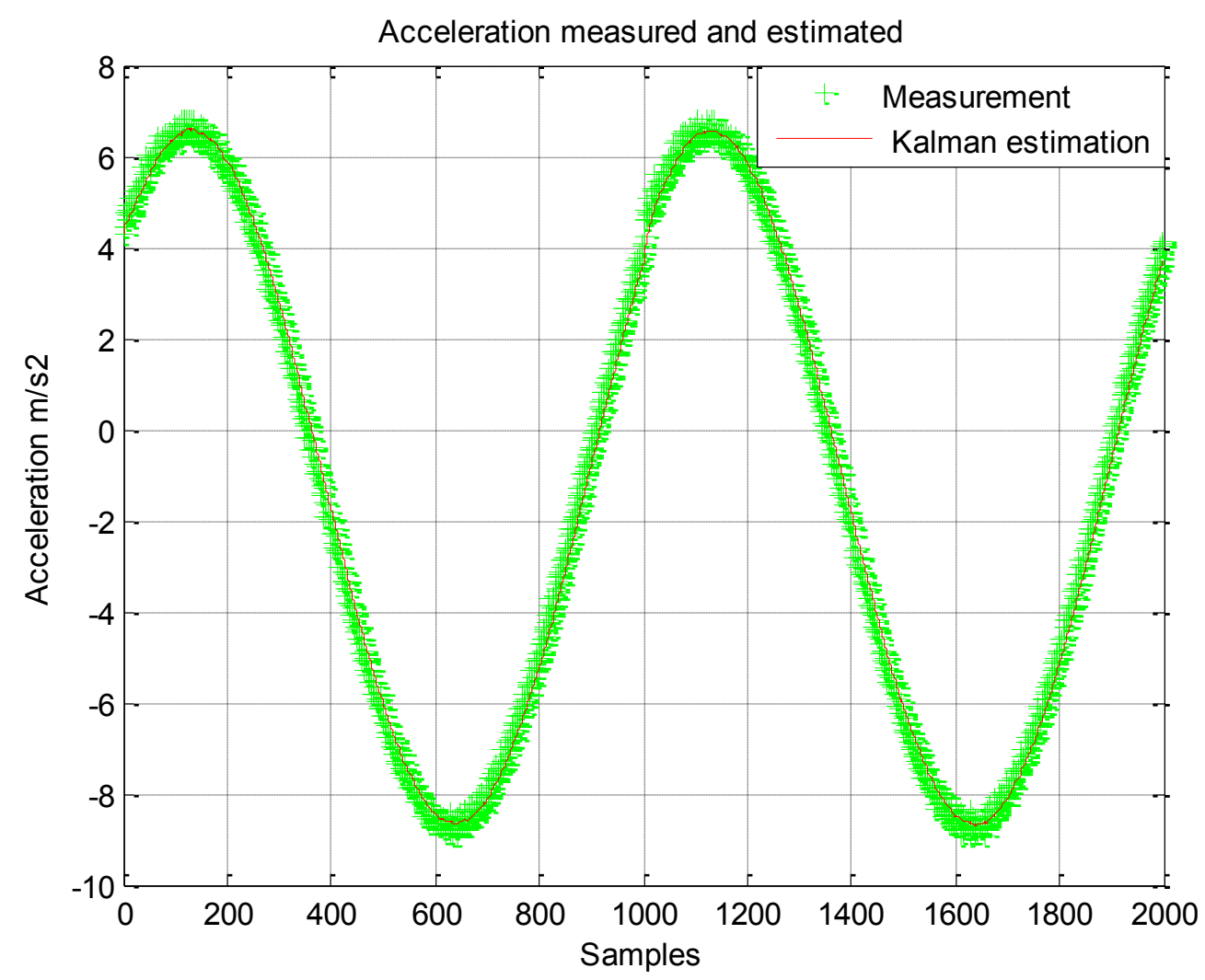

Figure 5.14 X Axis Acceleration Measurement

To estimate position, data was fused from both the $\mathrm{x}$ axes, for one revolution at $200 \mathrm{~Hz}$ as shown in the figure 5.14. A low sampling rate was chosen for rotational speed of $11 \mathrm{rpm}$. The true position was 0.62 meter. With double integration, unbounded error of nine meter was seen at half interval time. Kalman estimated the position as six meter. By using smoothing algorithm this error was reduced almost by four meters as shown by blue smoothed data in figure 5.15 . 


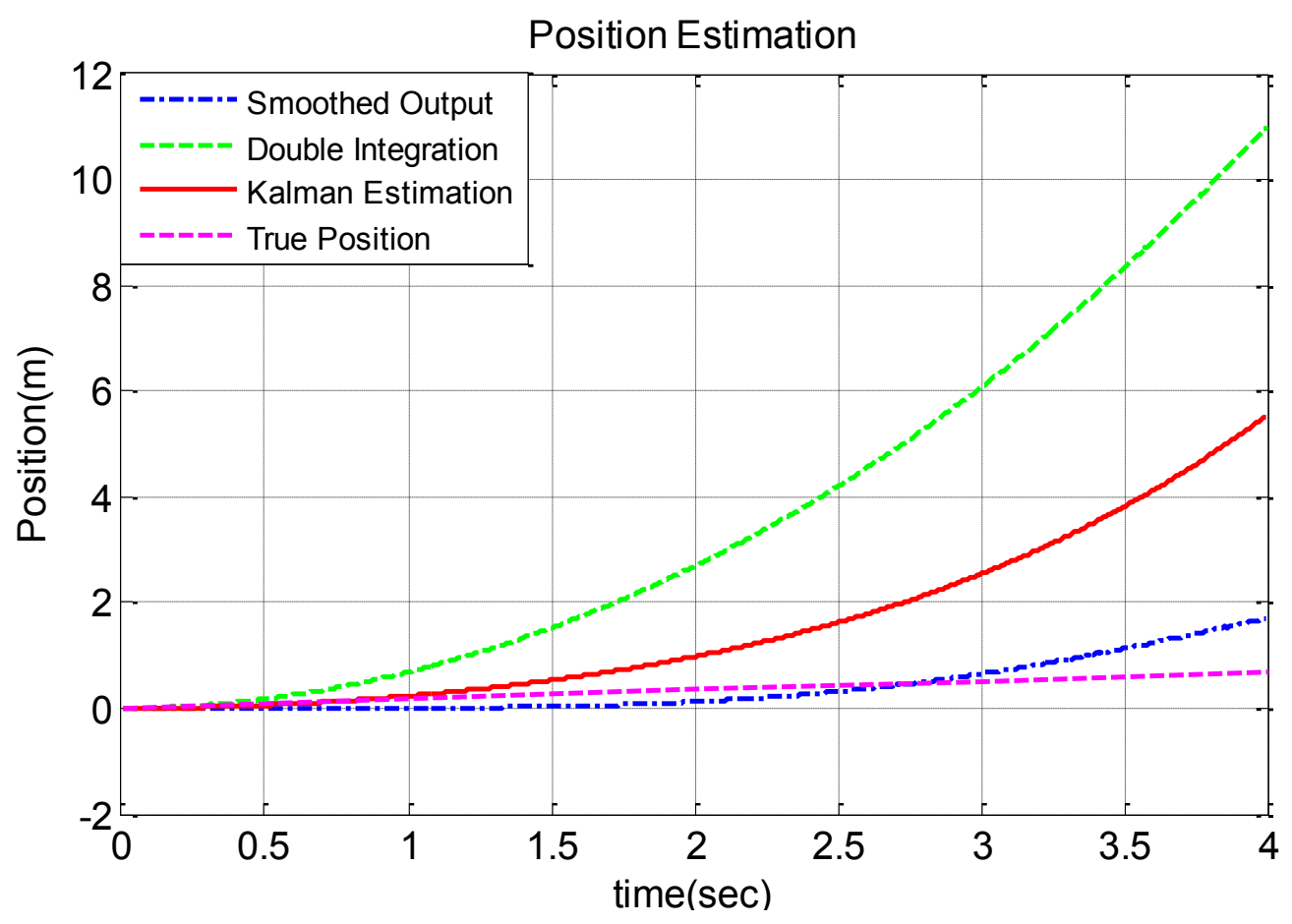

Figure 5.15 Position Estimation $-x_{1}$ and $x_{2}$ Data Fusion

Figure 5.16 shows the respective Kalman filter gain. As the filter converges to true value, Kalman gain decreases from 1 to 0.1 within first few seconds. By fusing all 4 axes data with Kalman filtering, estimated position and smoothed output was recorded at variable speed of 14, 16, 18 and $21 \mathrm{rpm}$. Figure 5.17 shows position error at different speeds after and before filtering at the end of one revolution. 


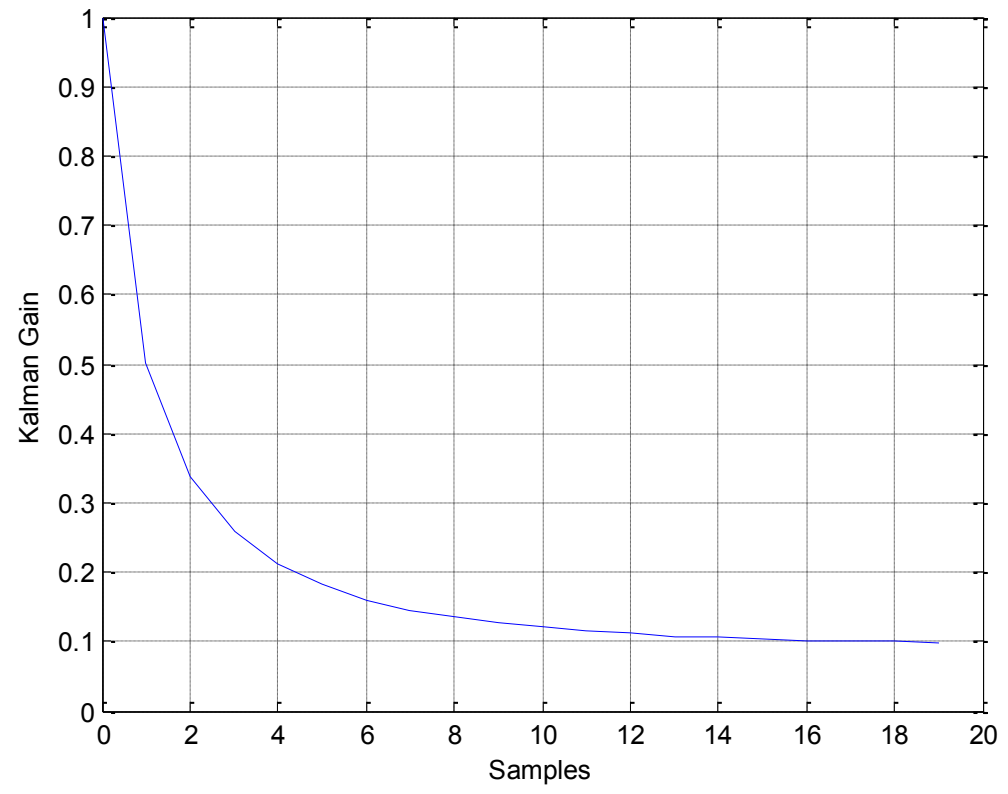

Figure 5.16 Kalman Gain

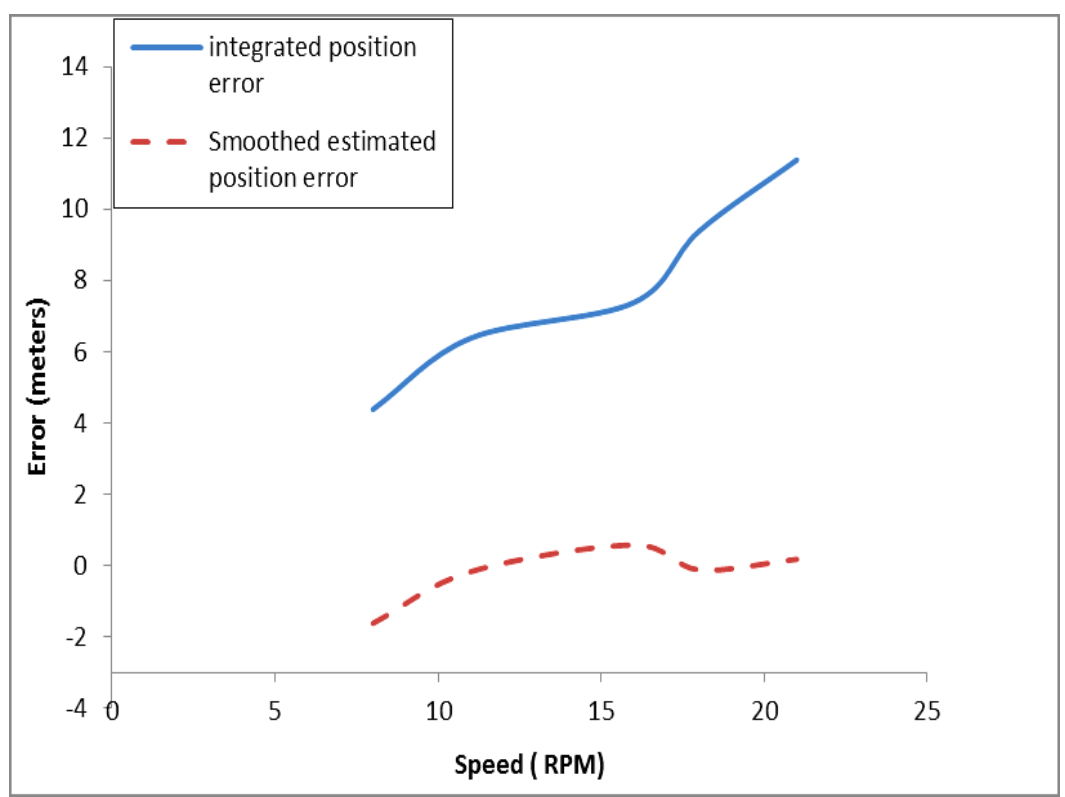

Figure 5.17 Unbounded Integrated Error and Smoothed Estimated Error at Different Speeds. 


\subsection{Tilt Measurement}

As explained in the previous section, angular acceleration can be recorded by gathering $y$ axis data from each sensor. Angular acceleration was calculated by using equation 4.55. Figure 5.18 shows raw noisy angular acceleration measurement and Kalman estimation at $11 \mathrm{rpm}$ and $200 \mathrm{~Hz}$ sampling rate.

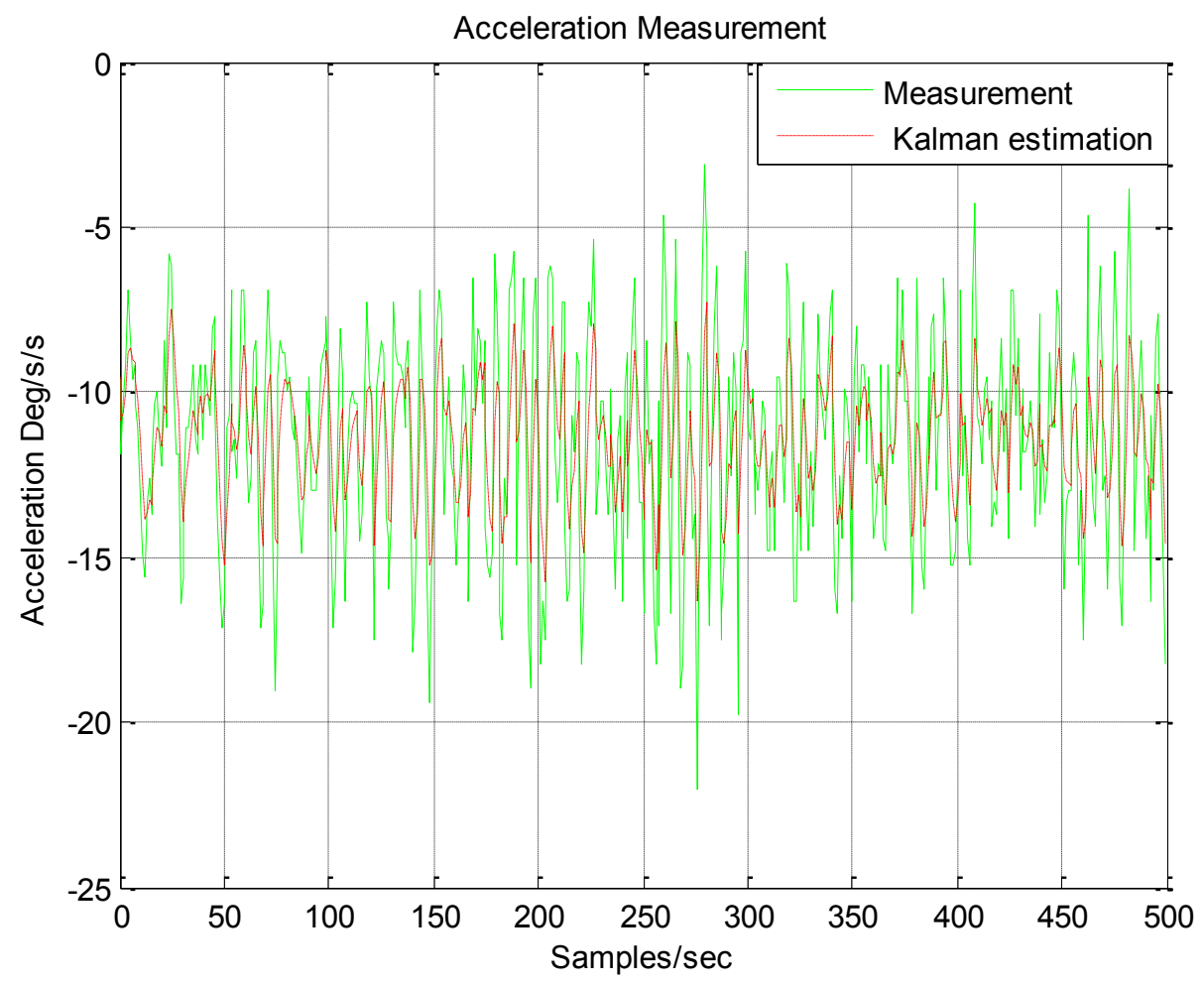

Figure 5.18 Acceleration and Kalman Estimation at Speed of $11 \mathrm{rpm}$

\subsection{Angular Position Estimation}

The angle can be determined by integrating angular velocity as given by the equation, 
$\theta=\omega\left(t_{i}-t_{1}\right)$ Where $t_{i}$ is the instantaneous time and $t_{1}$ is time recorded at first $+\mathrm{g}$ acceleration. Smoothed estimate showed an error of $-10^{\circ}$ in the angular measurement.

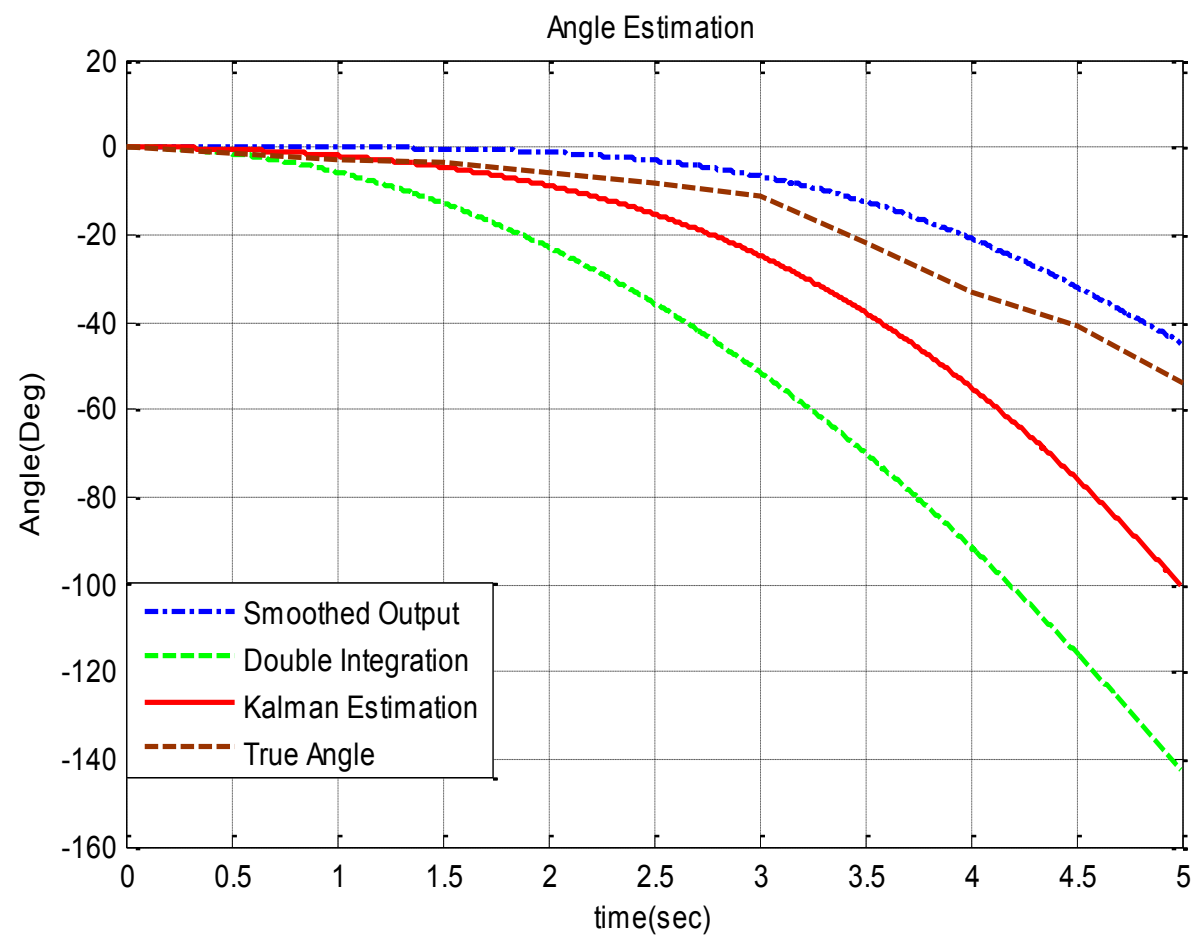

Figure 5.19 Angular Estimation

\subsection{Summary of Results}

It was observed that the integrated position error increases over time and the output was unacceptable for estimating correct position. In addition, it was noted that the Kalman filter successfully minimizes this error by combining all available sensory data and predicted position with the help of proposed bias drift error model. RTS smoother algorithm considerably reduces the position error by running the Kalman filter in backward direction. Similar behaviour was observed in angular acceleration measurement. 


\section{CHAPTER 6}

\section{Conclusion and Recommendations}

In this thesis, accelerometer error model was developed for testing, calibrating and evaluating performance of acceleration sensors for short term distance measurements. High Accuracy at low speed was achieved by using Kalman filter algorithm and it was further enhanced by using smoothing technique. Furthermore, the experimental results showed accelerometer as an effective, low cost, secondary sensor for short distance estimation in absence of other guided sensors.

\subsection{Conclusion}

This thesis work was primarily focused on understanding error sources in accelerometer sensor, calibrating and building proper error model scheme for optimum position estimation. This work was an attempt towards reducing sensor errors for more accurate navigation system. The contribution includes complete mechanical design for testing and calibrating adxl 335 accelerometer sensor using appropriate error model scheme. Tuning of Kalman filter by selecting optimum values of process noise covariance and measurement noise covariance played an important role in rapid estimation of angular velocity and position. As the process covariance value increases, prediction improves but at the cost of increase in error. The algorithm was verified and tested for several constant speed rotations. 
Various experiments were performed to validate error model for calibration. An average position error of $0.10 \mathrm{~m}$ had been seen after applying the error model in the Kalman algorithm. RTS algorithm was executed on the filtered data in offline mode.RTS smoother algorithm was introduced in the Kalman filter estimation for precise position estimation. It is observed that smoothing algorithm is more efficient than Kalman filter alone. It minimizes the mean square error at faster rate than the extended Kalman filter. The computational complexity of Kalman-smoother algorithm increases with increase in number of sensors used in data fusion. The smoothed output shows reduction in position error by $72 \%$. Accuracy could be further increased by including all the accelerometer error sources in an improved error model scheme.

\subsection{Recommendations}

In future research work, the smoothed output obtained by Kalman filter may be used to control DC motor speed as desired, with the use of force feedback. In that case, Kalman filter can be modeled as a controller for motor. A similar concept had been developed by [25] for controlling steering wheel. The nonlinear factors such as backlash and friction associated with motor can be modeled in the Extended Kalman Filter (EKF) for better prediction.

The calibration was performed specifically for ADXL 335 sensor. A parallel error model may be used for other manufacturers ICs as well. Calibration was performed on serial link with the help of rotary electrical connector to eliminate communication errors associated with wireless link. Wireless sensing techniques may be used to acquire data from sensors for real time applications. Furthermore, calibration and testing of other sensors such as gyro or optical encoders can be accomplished by using appropriate error modelling. Additionally, GPS and gyroscope sensors may be used along with accelerometer sensor by sensor fusion method to increase accuracy in the position estimation for various robotics and navigation applications. 


\section{Appendices}

\section{A.1. Sensor Fusion Two Dimensional Error Model}

The complete states of the system considering both the $2 \mathrm{D}$ axes, the number of states would be 14 as shown by equation,

$x_{k}=\left[\begin{array}{c}P_{x} \\ V_{x} \\ a_{x} \\ P_{y} \\ V_{y} \\ a_{y} \\ P_{x_{2}} \\ V_{x_{2}} \\ a_{x_{2}} \\ P_{x_{2}} \\ V_{y_{2}} \\ a_{y_{2}} \\ \varepsilon_{x} \\ \varepsilon_{y}\end{array}\right]$

Where, $P_{x_{1}}$ is $\mathrm{x}$ axis position state, $V_{x}$ is the velocity, assumed to be nearly constant and $a_{x}$ is the $\mathrm{x}$ axis acceleration for sensor $A_{1}$. Similar set of states are mentioned for sensor $A_{2}$. The transition matrix for above model can be written as, 


$$
A=\left[\begin{array}{cccc}
\Phi & 0 & 0 & 0 \\
0 & \Phi & 0 & 0 \\
0 & 0 & \Phi & 0 \\
0 & 0 & 0 & \Phi
\end{array}\right] \text { Where, } \Phi=\left[\begin{array}{cccc}
1 & \Delta t & 0.5 \Delta t^{2} & 0 \\
0 & 1 & \Delta t & 0 \\
0 & 0 & 1 & 0 \\
0 & 0 & 0 & \frac{\operatorname{Tax}}{\operatorname{Tax}+\Delta t}
\end{array}\right]
$$

The deterministic input will control the error model depending upon bias drift. Observation matrix contains acceleration measurement as, $C=\left[\begin{array}{llllllllllllll}0 & 0 & 1 & 0 & 0 & 1 & 0 & 0 & 1 & 0 & 0 & 1 & 0 & 0\end{array}\right]$

It takes all the acceleration measurement into consideration.

The process covariance matrix $\mathrm{Q}$ can be expressed as,

$Q=\left[\begin{array}{llll}\beta & 0 & 0 & 0 \\ 0 & \beta & 0 & 0 \\ 0 & 0 & \beta & 0 \\ 0 & 0 & 0 & \beta\end{array}\right]$

Where, $\quad \beta=\left[\begin{array}{cccc}\frac{W}{20} \Delta t^{5} & \frac{W}{8} \Delta t^{4} & \frac{W}{6} \Delta t^{3} & 0 \\ \frac{W}{8} \Delta t^{4} & \frac{W}{3} \Delta t^{3} & \frac{W}{2} \Delta t^{2} & 0 \\ \frac{W}{6} \Delta t^{3} & \frac{W}{2} \Delta t^{2} & W \Delta t & 0 \\ 0 & 0 & 0 & W \sigma_{\varepsilon}^{2}\end{array}\right]$

With spectral power density, $\mathrm{W}=1 \mathrm{rad} / \mathrm{sec}$. 
Thus the complete process model can be represented as,

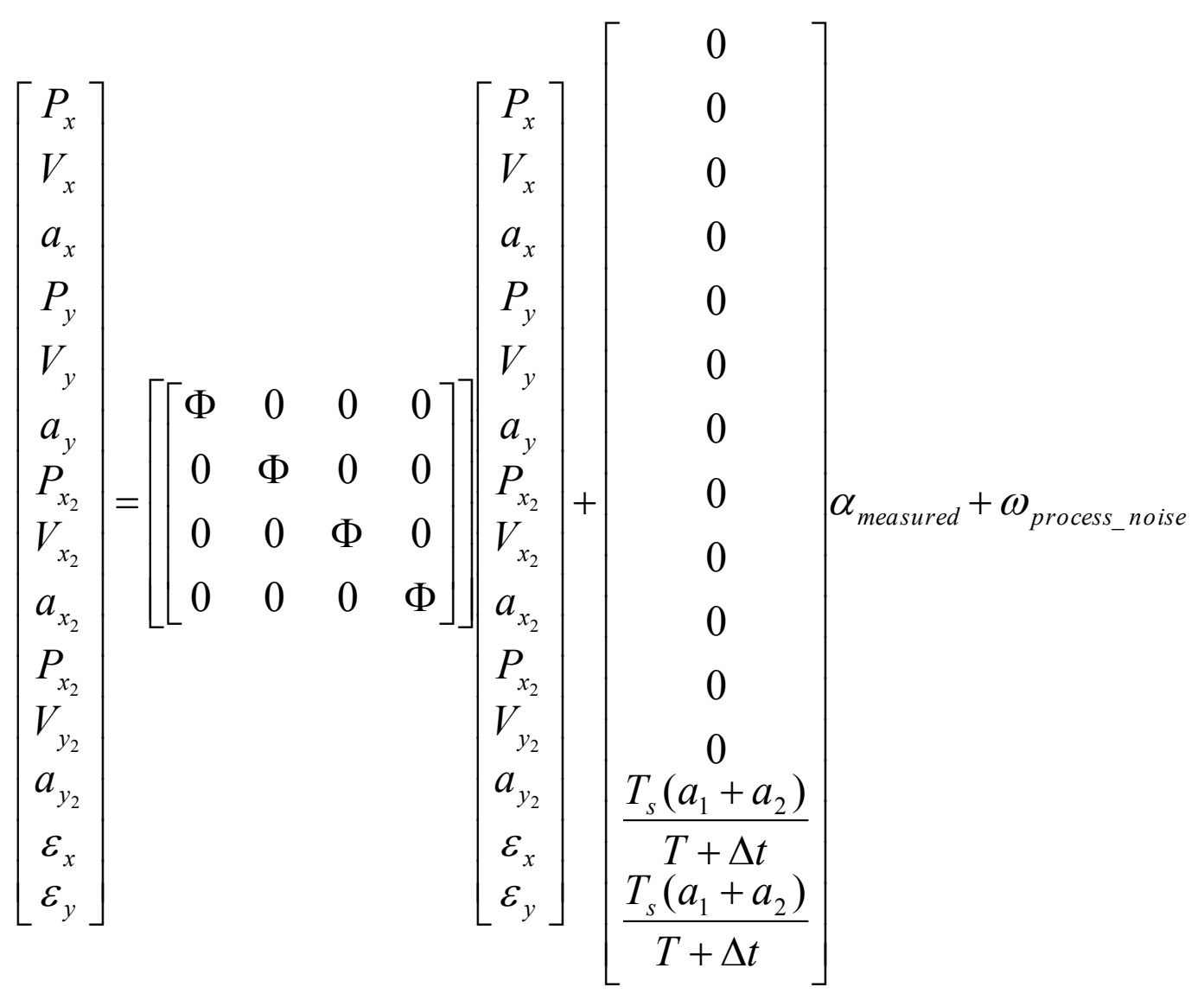

And observation model as,

$$
y_{k}=\left[\begin{array}{llllllllllllll}
0 & 0 & 1 & 0 & 0 & 1 & 0 & 0 & 1 & 0 & 0 & 1 & 0 & 0
\end{array}\right] x_{k}+v_{\text {measuremert_noise }}
$$




\section{A.2. Motor Speed Estimation}

To determine position of the rotating wheel, it's important to look at the basic equations.

Distance travelled per revolution:

Wheel diameter $=30 \mathrm{~cm}$

Perimeter value gives the distance traveled per revolution of wheel.

Perimeter $=\mathrm{pi}^{*}$ diameter $=3.14 * 30 \mathrm{~cm}=94.2 \mathrm{~cm} / \mathrm{rev}$

Angular Velocity $=$ rotation* perimeter of rotating wheel

Thus at constant rotational speed of $11 \mathrm{rpm}(11 / 60=0.1833)$,

$$
\begin{aligned}
\mathrm{W} & =0.1833 * 94.2 \\
& =17.26 \mathrm{Cm} / \mathrm{sec} \\
& =17.26 / 100=0.1726 \mathrm{~m} / \mathrm{s}
\end{aligned}
$$

There are $2 \pi$ (pi) radians (about 6.283185) in a complete circle.

- $1 \mathrm{rad}=360^{\circ} / 2 \pi=\sim 57.29578^{\circ}$

The radian is defined as the angle subtended at the center of a circle by an arc of circumference equal in length to the radius of the circle.

Converting angular velocity to other units

- $\quad 1 \mathrm{rad} / \mathrm{s}=9.55 \mathrm{r} / \mathrm{min}(\mathrm{rpm})=0.159 \mathrm{r} / \mathrm{s}(\mathrm{rps})$

Power $=\mathrm{dW} / \mathrm{dt}$ which is , rate of doing work.

Thus, Power $=\tau^{*} \omega$ where $\omega$ is the rotational Velocity.

Motor Torque $\tau$ stall is the maximum torque at $0 \mathrm{rpm}$. And $\omega_{\mathrm{n}}$ is the maximum speed when torque is 0 also known as no load speed. 
As we observe the equations we realised that the torque and rotational speed is inversely proportional to each other.

Power Requirements

Pout $=$ Force $*$ Distance/ Time

$=$ Force* $^{*}$ Velocity

$=$ Torque* Angular Velocity

Torque $=0.32 \mathrm{Nm}$

Expected velocity $=3 \mathrm{~m} / \mathrm{sec}=0.066 \mathrm{~m} / \mathrm{sec}$

Thus Pout $=0.32 * 0.066=0.0211$ watts

Table A.1 shows DC controller current settings and corresponding constant speed in RPM.

Table A.1 Motor controller RPM setting

\begin{tabular}{|l|l|}
\hline $\begin{array}{l}\text { DC controller Current } \\
\text { settings(A) }\end{array}$ & RPM \\
\hline 2 & 8 \\
\hline 3 & 11 \\
\hline 4 & 14 \\
\hline 5 & 16 \\
\hline 6 & 18 \\
\hline 7 & 21 \\
\hline
\end{tabular}




\section{A.2.1 Technical Specifications}

- Item: Dayton DC gear motor

- Type: Permanent Magnet

- Shaft Orientation: Parallel

- Nameplate RPM: 31

- Full Load Torque (In.-Lbs.): 220

- Voltage Rating: 90 VDC

- Overhung Load (Lb.): 150

- Input HP: $1 / 8$

- Gear Ratio: 58:1

- Full Load Amps: 1.27

- Enclosure: TENV

- Thermal Protection: None

- Ambient (C): 40

- Rotation: Reversible

- Mounting: All Position

- Length Less Shaft (In.): 10-1/8

- Shaft Dimensions (In.): $5 / 8$ x $11 / 2$

- Bearings: Needle Roller and Thrust Balls on Case, Ball on Motor

- Lubrication: Permanent Heavy Fluid Gear Oil

- Brushes: Externally Replaceable

- Gear Case: Die Cast Aluminum

- Gears: Hardened Steel

- Seals: On Input and Output Shaft 


\section{Gear Motor DC Speed Control}

Brand: Dayton, Model: 6A191SKU: 59093

Tech Specs

- HP: $1 / 35$ to $1 / 6$

- Voltage Output: 90

- Input Voltage: 115

- Speed Range: 15:1

- Speed Regulation: 10 to $30 \%$

- Mount Type: Direct, Eliminates Need to Run Long Wires from Control to Gearmotor, Offering a Streamlined, Compact Drive

- Includes: Convenient Speed Adjustment Right at the Gearmotor 


\section{A.3 Mechanical Design}

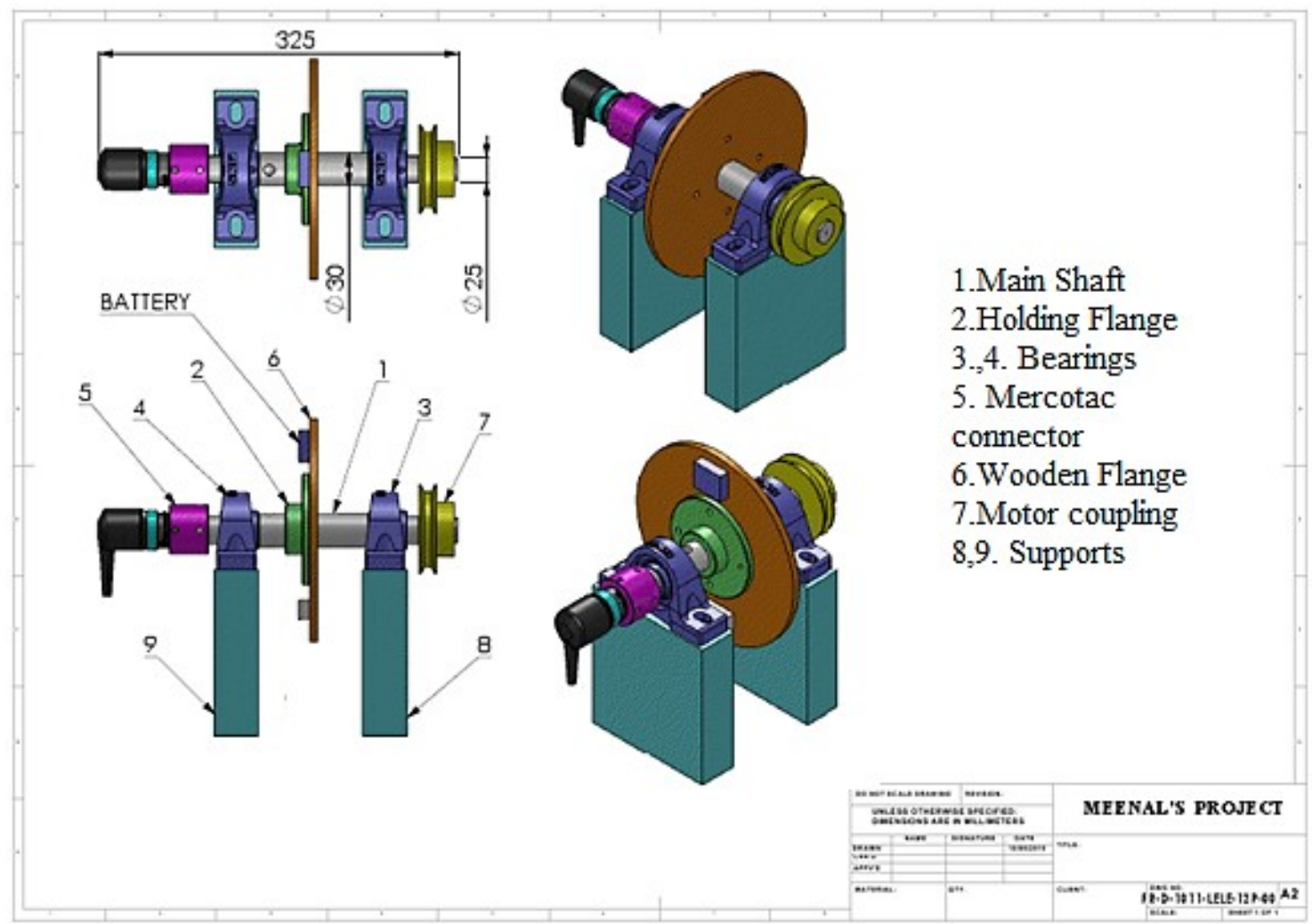




\section{Bibliography}

[1] U. Nehmzow "Mobile Robotics: A Practical Introduction" Springer-Verlag London Ltd.,pp 8-15, 2003.

[2] Robotics Research Group, "History of Robotics", Texas University, 2003, [Online]. Available: Robotics, http://www.robotics.utexas.edu/rrg/learn_more/history/ [Accessed: 22/8/2010]

[3] Bluebotics,"roboX, Interactive Tour Guide Robot" RoboX, 2001, [Online].Available: http://www.bluebotics.com/entertainment/RoboX/. [Accessed: 22/8/2010]

[4] J. Leonard and H. Durrant-Whyte. "Mobile robot localization by tracking geometric beacons". IEEE Tranactions on robot automation, vol 7(3), pp. 376-382, 1991.

[5] A.Singhal. "Issues in autonomous mobile robot navigation", Report, University of Rochester, pp. 3-12, 1997.

[6] J. Borenstein and L. Feng. "Measurement and correction of systematic odometry errors in mobile robots". IEEE Transactions on Robotics and Automation : A Publication of the IEEE Robotics and Automation Society. 12(6), pp. 869, 1996.

[7] L. Ojeda and J. Borenstein. "Methods for the reduction of odometry errors in overconstrained mobile robots." Autonomous Robots 16(3), pp. 273-286, 2004.

[8] M. Park and Y. Gao. "Error analysis and stochastic modeling of low-cost MEMS accelerometer." Journal of Intelligent and Robotic Systems: Theory and Applications 46(1), pp. 27-41, 2006. 
[9] P. Aggarwal. "A standard testing and calibration procedure for low cost MEMS inertial sensors and units." The Journal of Navigation, vol 61(02), pp. 323, 2008.

[10] M El-Diasty, A El-Rabbany and S Pagiatakis. " Temperature variation effects on stochastic characteristics for low-cost MEMS-based inertial sensor error" Measurement Scienct and Technology, vol 18, no. 11, 2007.

[11] G. Welch and G. Bishop. "An introduction to the Kalman filter" UNC-Chapel Hill, TR 95-041, July 24, 2006.

[12] M. S. Grewal and A. P. Andrews." Kalman Filtering: Theory and Practice using MATLAB John Wiley and Sons ,Inc. pp 457-470, 2008.

[13] D. Jonathan and H. Oliver, "Mobile Robot Navigation", surprise journal, 1997, [Online].Available:http://www.doc.ic.ac.uk/ nd/surprise_97/journal/vol4/jmd/.[Accessed $: 3 / 7 / 2010]$

[14] B. Barshan." Evaluation of a solid-state gyroscope for robotics applications." IEEE Transactions on Instrumentation and Measurement 44(1), pp. 61-67, 1995.

[15] B. Barshan, H. F. Durrant-Whyte "An inertial navigation system for a mobile robot." proceeding of 1993 IEEE International conference on intelligent robots and systems, pp 2244-2247, 1993.

[16] J. Borenstein, H. R. Everett and L. Feng. " Navigating Mobile Robots, Systems and Techniques, A.K.Peters Ltd., Wellesley,MA,pp. 23-26, 1996.

[17] C. L. Nelson and D. S. Fitzgerald. "Sensor fusion for intelligent alarm analysis." Security Technology, 30th Annual 1996 International Carnahan Conference., pp 1143$145,1996$. 
[18] J. Z. Sasiadek." Sensor fusion." Annual Reviews in Control ,vol 26(2), pp. 203-228, 2002.

[19] J. Bergeron and M. Looney. "Making MEMS accelerometers work in motion control" EETimes-Asia, pp 13-15, 2007.

[20] S. Jeon." Benefits of acceleration measurement in velocity estimation and motion control "Control Eng. Pract. vol 15(3), pp. 325, 2007.

[21] H. H. S. Liu and G. K. H. Pang. "Accelerometer for mobile robot positioning." Industry Applications, IEEE ,pp. 812-819, 2001.

[22] M. S. Miah, W. Gueaieb, M. A. Rahman and A. El Saddik." Autonomous deadreckoning mobile robot navigation system with intelligent precision calibration." Instrumentation and Measurement Technology Conference Proceedings. IMTC IEEE, pp. 5-9, 2007.

[23] K. S. Mostov, A. Soloviev and T. J. Koo. "Accelerometer based gyro-free multisensor generic inertial device for automotive applications." Intelligent Transportation System, ITSC 97. IEEE Conference on pp. 1047-1052, 1997.

[24] S. Nikbakht, M. Mazlom and A. Khayatian. "Evaluation of solid-state accelerometer for positioning of vehicle." Industrial Technology, ICIT IEEE International Conference pp. 729-733, 2005.

[25] J. Switkes " Using MEMS accelerometers to improve automobile handwheel state estimation for force feedback." American Society of Mechanical Engineers, Dynamic Systems and Control Division (Publication) DSC 73(2), pp. 1271-1278, 2004.

[26] Y. K. Thong, M. S. Woolfson, J. A. Crowe, B. Hayes-Gill and R. E. Challis. "Dependence of inertial measurements of distance on accelerometer noise." Measurement Science and Technology 13(8), pp. 1163-1172, 2002. 
[27] M. Park, "Error Analysis and Stochastic Modeling of MEMS based Inertial Sensors for Land Vehicle Navigation Applications" sensors, MDPI org ,pp 2240-2261, 2004.

[28] R. Carver and M. Looney. "Calibrate accelerometers for industrial applications." $E E$ Times-Asia, pp. 1-4, 2008.

[29] C. Acar and A. M. Shkel." Experimental evaluation and comparative analysis of commercial vaiable capacitance MEMS accelerometers" J Micromech Microengineering 13(5), pp. 634-645, 2003.

[30] Vector Nav "Inertial measurement unit and inertial navigation" 2009, [Online] Available: http:www.vectornav.com ,[Accessed: 30/6/2010].

[31] P. B. Reddy. "On stationary and nonstationary models of long term random errors of gyroscopes and accelerometers in an inertial navigation system." Presented at Decision and Control Including the 16th Symposium on Adaptive Processes and A Special Symposium on Fuzzy Set Theory and Applications, 1977.

[32] H. Seidel, R. Burghardt, B. Hartmann, R. Gottinger, M. Aikele, K. Kapser, W. Gessner and J. Valldorf, "A Low-g Accelerometer for Inertial Measurement Units," VDIBuch, Springer Berlin Heidelberg, vol. 978-3-540-76988-0, pp. 349-358, 2003.

[33] W. T. Ang, P. K. Khosla and C. N. Riviere." Nonlinear regression model of aLow-g MEMS accelerometer". Sensors Journal, IEEE 7(1), pp. 81-88, 2008.

[34] G. Aslan and A. Saranli, "CHARACTERIZATION AND CALIBRATION OF MEMS INERTIAL MEASUREMENT UNITS,"16 ${ }^{\text {th }}$ European Signal Processing Conference, Lausanne, Switzerland,pp 1-5, 2008.

[35] Analog devices,"ADXL335", 2008, [Online]. Available: http://www.analog.com /static/importedfiles/data_sheets/ADXL335.pdf. [Accessed:16/2/2010]. 
[36] C. Bouten "Triaxial accelerometer and portable data processing unit for the assessment of daily physical activity." IEEE Trans. Biomed. Eng. 44(3), pp. 136-137, 1997.

[37] M. Stakkeland, G. Prytz, W. E. Booij and S. T. Pedersen. "Characterization of accelerometers using nonlinear kalman filters and position feedback." IEEE Transactions on Instrumentation and Measurement 56(6), pp. 2698-2704, 2007.

[38] S. Bogatin."Evaluation of linear kalman filter processing geodetic kinematic measurements. Measurement 41(5), pp. 561-568, 2008.

[39] J. Gao." Research on an inertial positioning system for a parallel kinematic machine." Mechatronics vol 15(1), pp. 1-7, 2005.

[40] J. Gao, P. Webb and N. Gindy." Evaluation of a low-cost inertial dynamic measurement system " Robotics, Automation and Mechatronics, pp. 5-10, 2008.

[41] The MEMS and Nanotechnology Exchange Organization. "What is MEMS "2001, [Online].Available: http://www.mems-exchange.org/MEMS/what is MEMS [Accessed: $3 / 12 / 2009]$.

[42] Clare, "What is MEMS accelerometer" Nano glass 2001, [Online]. Available: http://nanogloss.com/category/mems. [Accessed: 15/9/2009].

[43] IEEE Aerospace and Electronic Systems Society. Gyro and Accelerometer Panel. and Institute of Electrical and Electronics Engineers. 1999, IEEE Standard Specification Format Guide and Test Procedure for Linear, Single-Axis, Nongyroscopic Accelerometers .

[44] D.W.Marquardt "An algorithm for least-squares estimation of nonlinear parameters" Journal of the Society for Industrial and Applied Mathematics, vol 11,no.2, pp431-441, 1963. 
[45] Mercotac Inc."Electrical Connectors" 2001, [Online]. Available:http:www.mercotac. com /html/technicalinfo.html. [Accessed: 29/2/2009].

[46] X. FANG, J. ZHAO and Y. HU. " Tests and error analysis of a self-positioning shearer operating at a man less working face. "Mining Science and Technology (China) 20(1), pp. 53-58, 2010.

[47] G. Welch and G. Bishop. "An introduction to the Kalman filter" UNC-Chapel Hill,TR 95-041,July 24, 2006.

[48] H. Symon , "Kalman filtering and neural networks," John Wiley \& Sons, Inc., 2001, pp. 1-20, 2001.

[49] S. Dan, "Optimal state estimation" NewJersy: John Wiley \& Sons, Inc., 2006, pp. $123,2006$.

[50] A. Gelb, J. Kasper, R. Nash, C. Price and A. Sutherland."Applied Optimal Estimation" The Analytic Sciences Corporation, pp156-167, 1974.

[51] H. E. Rauch, F. Tung and C. T. Striebel. "Maximum likelihood estimates of linear dynamic systems", AIAA J. 3, pp. 1445-1450, 1965. 